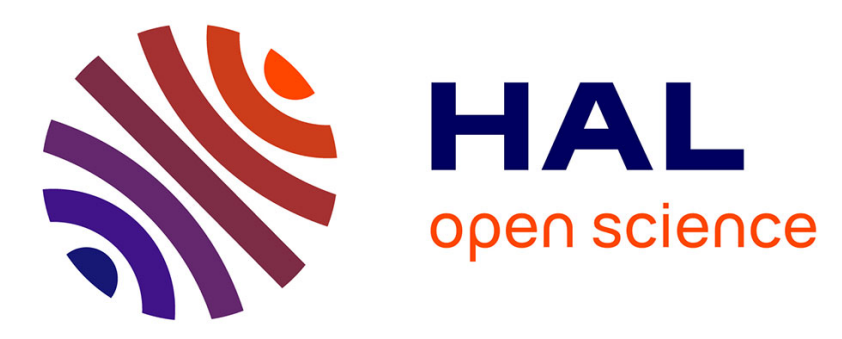

\title{
Mathematical analysis and 2-scale convergence of a heterogeneous microscopic bidomain model
}

\author{
Annabelle Collin, Sébastien Imperiale
}

\section{To cite this version:}

Annabelle Collin, Sébastien Imperiale. Mathematical analysis and 2-scale convergence of a heterogeneous microscopic bidomain model. Mathematical Models and Methods in Applied Sciences, 2018, 10.1142/S0218202518500264 . hal-01759914

\section{HAL Id: hal-01759914 \\ https://hal.inria.fr/hal-01759914}

Submitted on 6 Apr 2018

HAL is a multi-disciplinary open access archive for the deposit and dissemination of scientific research documents, whether they are published or not. The documents may come from teaching and research institutions in France or abroad, or from public or private research centers.
L'archive ouverte pluridisciplinaire HAL, est destinée au dépôt et à la diffusion de documents scientifiques de niveau recherche, publiés ou non, émanant des établissements d'enseignement et de recherche français ou étrangers, des laboratoires publics ou privés. 


\title{
Mathematical analysis and 2-scale convergence of a heterogeneous microscopic bidomain model
}

\author{
Annabelle Collin Sébastien Imperiale
}

Accepted M3AS 2018

DOI: S0218202518500264

\begin{abstract}
The aim of this paper is to provide a complete mathematical analysis of the periodic homogenization procedure that leads to the macroscopic bidomain model in cardiac electrophysiology. We consider space-dependent and tensorial electric conductivities as well as space-dependent physiological and phenomenological non-linear ionic models. We provide the nondimensionalization of the bidomain equations and derive uniform estimates of the solutions. The homogenization procedure is done using 2-scale convergence theory which enables us to study the behavior of the non-linear ionic models in the homogenization process.
\end{abstract}

\section{Introduction}

Cardiac electrophysiology describes and models chemical and electrical phenomena taking place in the cardiac tissue. Given the large number of related pathologies, there is an important need for understanding these phenomena. As illustrated in Figure 1, there are two modeling scales in cardiac electrophysiology. The modeling at the microscopic scale aims at producing a detailed description of the origin of the electric wave in the cells responsible for the heart contraction. The modeling at the macroscopic scale - deduced from the microscopic one using asymptotic techniques - describes the propagation of this electrical wave in the heart.

One of the most popular mathematical models in cardiac electrophysiology is the bidomain model, introduced by [62] and described in detail in the reference textbooks [56], [59] and [52]. At the microscopic scale, this model is based upon the description of electrical and chemical quantities in the cardiac muscle. The latter is segmented into the intra- and the extra-cellular domains - hence the name of the model. These two domains are separated by a membrane where electric exchanges occur. A simple variant found in the literature comes from an electroneutrality assumption - justified by an asymptotic analysis - applied to the Nernst-Planck equations, see for example [41] and [40]. This variant leads to partial differential equations whose unknowns are intra- and extra-cellular electric potentials coupled with non linear ordinary differential equations called ionic models at the membrane. They represent the transmembrane currents and other cellular ionic processes. Many non-linear ionic models exist in the literature and can be classified into two categories: physiological models, see for instance $[28,46,35,19]$ and phenomenological models, see for example $[39,42,23]$. See also [30] and [56] as reference textbooks on the matter. The choice of an adapted model is based on the type of considered cardiac cells (ventricles, atria, Purkinje fibers, ... ) but also on the desired algorithm complexity (in general phenomenological models are described with less parameters). From the mathematical standpoint, existence and uniqueness analysis for different ionic models is given in [17,63].

A homogenization procedure allows for the deduction of the macroscopic behaviors from the microscopic ones and leads to the equations of the macroscopic bidomain model. Concerning the mathematical point of view, this homogenization procedure is given formally 
in [43] or more recently in $[17,53]$. In [6] and [50], it is proven using $\Gamma$-convergence. The existence and the uniqueness of a solution for the bidomain model at the macroscopic scale have been studied for different ionic models in the literature [17, 57, 64, 8, 10, 11].

The aim of this paper is to fill a gap in the literature by providing a complete mathematical analysis based on 2-scale convergence theory of the homogenization procedure that leads to the macroscopic bidomain model. Our analysis is exhaustive in the sense that we provide existence and uniqueness results, nondimensionalization of the equations and 2-scale convergence results - in particular, for the non-linear terms supported on the membrane surface in the same mathematical framework. To anticipate meaningful modeling assumptions, we consider that the electric conductivities are tensorial and space varying at the microscopic scale. We also consider ionic models of both types (physiological and phenomenological) that may vary smoothly in space (in order to consider ventricular or atrial cells for instance). We carefully introduce the various standard assumptions satisfied by the ionic terms and discriminate the models compatible with our analysis. We are convinced that this work will further allow the analysis of more complex models by laying the ground of the bidomain equations 2-scale analysis. More precisely, among the modeling ingredients that could fit in our context, one could consider: heterogeneous concentrations of ionic species inside the cells, influences of heart mechanical deformations [53, 24, 18], gap junctions [27] and the cardiac microscopic fiber structure in the context of local 2-scale convergence [12, 51].

The paper is organized as follows.

- In Section 2, we describe the considered heterogeneous microscopic bidomain model and review the main ionic models. Depending on how they were derived, we organize them into categories. This categorization is useful for the existence (and uniqueness) analysis.

- Although it is not the main focus of the article, we present - in Section 3 - existence and uniqueness results of the heterogeneous microscopic bidomain model. The proof - given in Appendix 5 -uses the Faedo-Galerkin approach. The originality of the proposed strategy is the reformulation of the microscopic equations as a scalar reaction-diffusion problem, see Section 3.1. Such an approach is inspired by the macroscopic analysis done in [11] and from the analysis of an electroporation model given in [29]. Then, before stating the existence and uniqueness theorems of Section 3.3, we present and discuss - see Section 3.2 - in detail all the mathematical assumptions required. Finally, in Section 3.4, we explain how the solutions of our original problem can be recovered by a post-processing of the scalar reaction-diffusion problem solutions.

- In Section 4, the homogenization process of the heterogeneous microscopic bidomain model is given. It relies on the underlying assumption that the medium is periodic and uses the 2-scale convergence theory (see [3]). In preliminary steps, we provide a nondimensionalization of the bidomain equations, see Section 4.1. In order to mathematically analyze the micro- and macroscopic scales of the model and to consider the time-dependence of the system, we develop - in Section 4.2 - adapted uniform estimates. Our strategy can be extended to other problems as for instance the study of cell electroporation. Then the 2-scale convergence theory is applied in order to obtain the macroscopic bidomain equations. The analysis is done in three steps:

- In Section 4.3, we give the mathematical framework required for the 2-scale convergence. Among the standard results of the 2-scale convergence theory that we recall, we provide less standard properties by giving 2 -scale convergence results on surfaces.

- In Section 4.4 the 2-scale convergence is used and the limit homogenized problem is given. It corresponds to a 2-scale homogenized model. A specific care is taken for the convergence analysis of the non-linear terms and it represents one of the most technical point of the presented approach.

- Finally, in Section 4.5, the two-scale homogenized model is decoupled and a macroscopic bidomain equation is recovered.

Concerning the 2-scale convergence analysis, we also refer the reader to the work done in $[6,50]$ in which a proof of the homogenization process is proposed using $\Gamma$-convergence for a specific ionic model. This technique is well suited when the model is naturally 


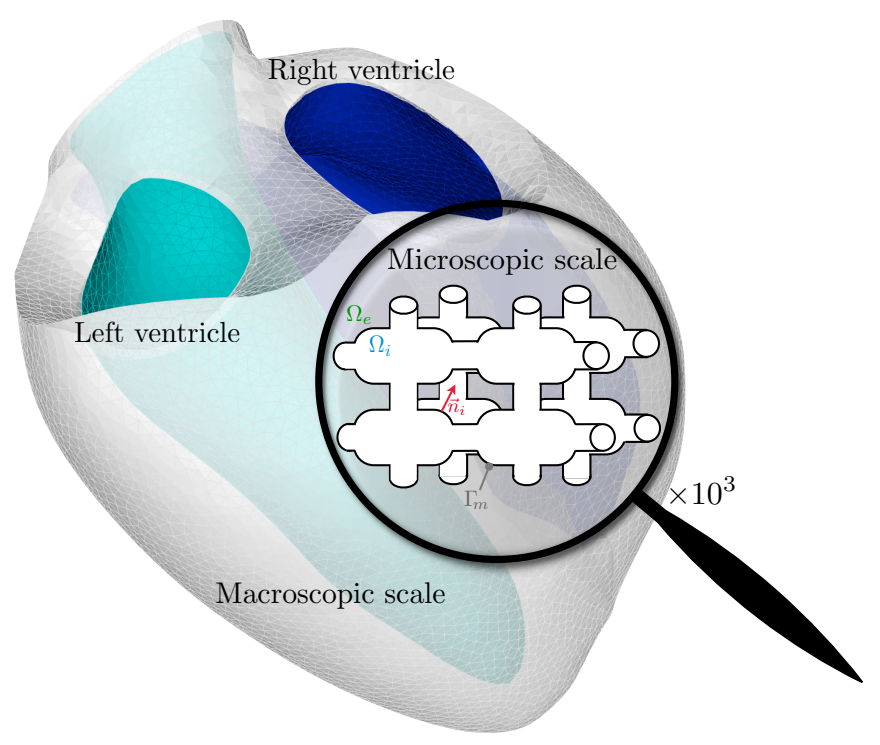

Figure 1: Cartoon of the considered domain at the microscopic scale and the macroscopic scale.

described as the minimization of a convex functional which is not the case for all the physiological ionic models.

\section{Microscopic bidomain model}

In this section, we give a short description of the heterogeneous microscopic bidomain model and the considered ionic models.

The cardiac muscle is decomposed into two parts. We denote by $\Omega \subset \mathbb{R}^{3}$ the volume of the heart, $\Omega_{i}$ the intracellular region and $\Omega_{e}$ the extracellular region. Physiologically, the cells are connected by many gap junctions therefore, geometrically, we assume that $\Omega_{i}$ and $\Omega_{e}$ are two connected domains with Lipschitz boundary verifying

$$
\bar{\Omega}=\bar{\Omega}_{e} \cup \bar{\Omega}_{i} \text { and } \Omega_{e} \cap \Omega_{i}=\emptyset .
$$

The subscripts $i$ and $e$ are used to distinguish the intra- and extracellular quantities, respectively, and $\alpha$ to refer to either of them indifferently. We suppose that the membrane $\Gamma_{m}=\partial \Omega_{e} \cap \partial \Omega_{i}$ is regular and non-empty. We define $\vec{n}_{i}$ and $\vec{n}_{e}$ as the unit normal vectors pointing from $\Omega_{i}$ and $\Omega_{e}$, respectively, to the exterior. The following microscopic bidomain model is studied for time $t \in[0, T]$

$$
\left\{\begin{array}{cccc}
\vec{\nabla}_{\vec{x}} \cdot\left(\overrightarrow{\vec{\sigma}}_{\alpha} \vec{\nabla}_{\vec{x}} u_{\alpha}\right) & = & 0 & \Omega_{\alpha}, \\
\overrightarrow{\vec{\sigma}}_{i} \vec{\nabla}_{\vec{x}} u_{i} \cdot \vec{n}_{i} & = & \overrightarrow{\vec{\sigma}}_{e} \vec{\nabla}_{\vec{x}} u_{e} \cdot \vec{n}_{i} & \Gamma_{m}, \\
-\overrightarrow{\vec{\sigma}}_{i} \vec{\nabla}_{\vec{x}} u_{i} \cdot \vec{n}_{i} & = & C_{m} \frac{\partial V_{m}}{\partial t}+I_{i o n}^{t o t} & \Gamma_{m}, \\
V_{m} & = & u_{i}-u_{e} & \Gamma_{m},
\end{array}\right.
$$

where $u_{i}$ and $u_{e}$ are electric potentials, $C_{m}$ the membrane capacitance and $I_{i o n}^{\text {tot }}$ an electrical current depending on ionic activities at the membrane. The conductivities $\overrightarrow{\vec{\sigma}}_{\alpha}$ are assumed to be tensorial and depend on $\vec{x}$ in order to take various modeling assumptions into account. For example, this general form of the conductivities allows us to consider: the dependence of ionic concentrations (remark that a first approximation is to consider space-wise constant ionic concentrations); the heart mechanical deformations [24, 18] or a complex model of gap junctions (see [27]). In order to close the problem, we need to prescribe adequate boundary 
conditions on $\partial \Omega$, the external boundary of the domain. We assume that no electric current flows out of the heart

$$
\vec{\sigma}_{\alpha} \vec{\nabla}_{\vec{x}} u_{\alpha} \cdot \vec{n}_{\alpha}=0, \quad \partial \Omega_{\alpha} \cap \partial \Omega .
$$

Finally, one can observe that Equations (2) and (3) define $u_{i}$ or $u_{e}$ up to the same constant. Therefore, we choose to impose

$$
\int_{\Gamma_{m}} u_{e} d \gamma=0
$$

We now describe the term $I_{\text {ion }}^{\text {tot }}$ which appears in (2). In terms of modeling, action potentials are produced as a result of ionic currents that pass across the cell membrane, triggering a depolarization or repolarization of the membrane over time. The currents are produced by the displacement of ionic species across the membrane through ionic channels. The channels open and close in response to various stimuli that regulate the transport of ions across the membrane. The cell membrane can be modeled as a combined resistor and capacitor,

$$
C_{m} \frac{\partial V_{m}}{\partial t}+I_{i o n}^{t o t}
$$

The ionic current $I_{i o n}^{\text {tot }}$ is decomposed into two parts, $I_{i o n}^{\text {tot }}=I_{i o n}-I_{a p p}$, where the term $I_{a p p}$ corresponds to the external stimulus current. Historically, the first action potential model is the Hodgkin-Huxley model [28]. In order to understand the complexity of physiological models, we give a brief description of this model - the most important model in all of the physiological theory see [30] - originally formulated for neurons. The transmembrane current $I_{i o n}$ proposed by the Hodgkin-Huxley model is $I_{i o n}=I_{N a}+I_{K}+I_{l}$, where $I_{N a}$ is the sodium current, $I_{K}$ the potassium current and $I_{l}$ the leakage current which concerns various and primarily chloride ions. The currents are determined for $k=N a, K, l$ by $I_{k}=g_{k}\left(V_{m}-E_{k}\right)$, where $g_{k}$ is the conductance and $E_{k}$, the equilibrium voltage. The conductance $g_{l}$ is supposed to be constant and the other conductances are defined by

$$
g_{N a}=m^{3} h \bar{g}_{N a}, \quad g_{K}=n^{4} \bar{g}_{K},
$$

where $\bar{g}_{N a}$ and $\bar{g}_{K}$ are the maximal conductances of the sodium and potassium currents, respectively. The dimensionless state variables $m, n$ and the inactivation variable $h$ satisfy the following ordinary differential equations

$$
\partial_{t} w=\alpha_{w}\left(V_{m}\right)(1-w)-\beta_{w}\left(V_{m}\right) w, w=m, n, h,
$$

where $\alpha_{w}$ and $\beta_{w}$ are the voltage-dependent rate constants which control the activation and the inactivation of the variable $w$. In Chapter 4 of [30], $\alpha_{w}$ and $\beta_{w}$ both have the following form

$$
\frac{C_{1} e^{\left(V_{m}-V_{0}\right) / C_{2}}+C_{3}\left(V_{m}-V_{0}\right)}{1+C_{4} e^{\left(V_{m}-V_{0}\right) / C_{5}}},
$$

where $C_{i}, i=1, \cdots, 5$ and $V_{0}$ are the model parameters. An adaptation of the HodgkinHuxley model to the cardiac action potential was suggested by D. Noble in 1962 [46]. Many physiological models have been proposed ever since: for the ventricular cells [35, 60, 61, 26, $48]$ and for the atrial cells [26, 19, 47, 36, 31, 25, 65]. We refer for example to [56] for a 2004 survey.

All the cited models are physiological models. Other models - called phenomenological models - are approximations of the ionic channels behavior. These models are intended to describe the excitability process with a lower complexity. With only one (or few) additional variable(s) denoted by $w$ and called the state variable(s) - and then only one (or few) ordinary differential equation(s) - these models are able to reproduce the depolarization and/or the repolarization of the membrane. The FitzHugh-Nagumo (FHN) model [23, 42], the Roger and McCulloch model [55] and the Aliev and Panfilov model [2] can be written as follows

$$
\left\{\begin{array}{c}
I_{i o n}\left(V_{m}, w\right)=k\left(V_{m}-V_{\text {min }}\right)\left(V_{m}-V_{\text {max }}\right)\left(V_{m}-V_{\text {gate }}\right)+f_{2}\left(V_{m}\right) w \\
\partial_{t} w+g\left(V_{m}, w\right)=0
\end{array}\right.
$$


with

$$
g\left(V_{m}, w\right)=\delta\left(\gamma g_{1}\left(V_{m}\right)+w\right)
$$

and where $\delta, \gamma, k$ and $V_{\text {gate }}$ are positive constants. The parameters $V_{\min }$ and $V_{\max }$ are reasonable potential ranges for $V_{m}$. The functions $f_{2}$ and $g_{1}$ (see Assumption 7 for the notational choice) depend on the model. A more widely phenomenological accepted model for ventricular action potential is the Mitchell-Schaeffer model presented in [39],

$$
\left\{\begin{array}{c}
I_{\text {ion }}=\frac{w}{\tau_{\text {in }}} \frac{\left(V_{m}-V_{\min }\right)^{2}\left(V_{m}-V_{\max }\right)}{V_{\max }-V_{\min }}-\frac{V_{m}-V_{\min }}{\tau_{\text {out }}\left(V_{\max }-V_{\min }\right)}, \\
\partial_{t} w+g\left(V_{m}, w\right)=0,
\end{array}\right.
$$

with

$$
g\left(V_{m}, w\right)=\left\{\begin{array}{cl}
\frac{w}{\tau_{\text {open }}}-\frac{1}{\tau_{\text {open }}\left(V_{\text {max }}-V_{\text {min }}\right)^{2}} & \text { if } V_{m} \leq V_{\text {gate }}, \\
\frac{w}{\tau_{\text {close }}} & \text { if } V_{m}>V_{\text {gate }},
\end{array}\right.
$$

and with $\tau_{\text {open }}, \tau_{\text {close }}, \tau_{\text {in }}, \tau_{\text {out }}$ and $V_{\text {gate }}$, positive constants. Due to its lack of regularity, the mathematical analysis of this model is complicated. A straightforward simplification consists in using a regularized version of this model. Following [20], it reads

$$
g\left(V_{m}, w\right)=\left(\frac{1}{\tau_{\text {close }}}+\frac{\tau_{\text {close }}-\tau_{\text {open }}}{\tau_{\text {close }} \tau_{\text {open }}} h_{\infty}\left(V_{m}\right)\right)\left(w-\frac{h_{\infty}\left(V_{m}\right)}{\left(V_{\text {max }}-V_{\text {min }}\right)^{2}}\right)
$$

and

$$
h_{\infty}\left(V_{m}\right)=-\frac{1}{2} \tanh \left(\frac{V_{m}-V_{\min }}{\left(V_{\text {max }}-V_{\text {min }}\right) \eta_{\text {gate }}}-\frac{V_{\text {gate }}}{\eta_{\text {gate }}}\right)+\frac{1}{2},
$$

where $\eta_{\text {gate }}$ is a positive constant. This regularized version is considered in what follows in order to prove mathematical properties of the bidomain problem.

Remark 1. We consider non-normalized versions of the ionic models. For the considered phenomenological models, we expect to have

$$
V_{\min } \leq V_{m} \leq V_{\max }
$$

although it can not be proven without strong assumptions on the source term. Concerning the gating variable, we expect to have

$$
0 \leq w \leq 1 \text { for FHN like models }
$$

and, following a choice commonly done in the literature of the Mitchell-Schaeffer model, we expect to have

$$
0 \leq w \leq \frac{1}{\left(V_{\max }-V_{\min }\right)^{2}} \text { for the Mitchell-Schaeffer model (9, 10). }
$$

This last inequality can be proven (see Assumption 10 below and the proof of Lemma 2 in Appendix). Finally for all physiological models, we expect some bounds - from below and above - on the gating variables(s) as natural consequences of the structure of Equation (6). Note that in what follows, we consider the bidomain equations with only one gating variable $w$. All the results presented below can be extended to the case where the ionic term $I_{i o n}$ depends on several gating variables.

In the next section, the following microscopic bidomain model is studied (it corresponds to System (2) coupled with an ionic model and with the boundary conditions (3) and (4)), 
for all time $t \in[0, T]$,

$$
\left\{\begin{array}{cccc}
\vec{\nabla}_{\vec{x}} \cdot\left(\vec{\sigma}_{\alpha} \vec{\nabla}_{\vec{x}} u_{\alpha}\right) & = & 0 & \Omega_{\alpha}, \\
\vec{\sigma}_{i} \vec{\nabla}_{\vec{x}} u_{i} \cdot \vec{n}_{i} & = & \vec{\sigma}_{e} \vec{\nabla}_{\vec{x}} u_{e} \cdot \vec{n}_{i} & \Gamma_{m}, \\
-\vec{\sigma}_{i} \vec{\nabla}_{\vec{x}} u_{i} \cdot \vec{n}_{i} & = & C_{m} \frac{\partial V_{m}}{\partial t}+I_{i o n}^{t o t}\left(V_{m}, w\right) & \Gamma_{m}, \\
V_{m} & = & u_{i}-u_{e} & \Gamma_{m}, \\
\partial_{t} w & = & -g\left(V_{m}, w\right) & \Gamma_{m}, \\
\vec{\sigma}_{i} \vec{\nabla}_{\vec{x}} u_{i} \cdot \vec{n}_{i} & = & 0 & \partial \Omega_{i} \cap \partial \Omega, \\
\overrightarrow{\vec{\sigma}}_{e} \vec{\nabla}_{\vec{x}} u_{e} \cdot \vec{n}_{e} & = & 0 & \partial \Omega_{e} \cap \partial \Omega . \\
\int_{\Gamma_{m}} u_{e} d \gamma & = & 0 . &
\end{array}\right.
$$

\section{Analysis of the microscopic bidomain model}

In this section, the analysis (existence and uniqueness) of the heterogeneous microscopic bidomain model presented in Section 2 is proposed.

As explained before, we assume that $\Omega_{i}$ and $\Omega_{e}$ are connected sets and that they have a Lipschitz boundary. Our analysis involves the use of standard $L^{p}$ Banach spaces and $H^{s}$ Hilbert spaces. Apart from the use of $(\cdot, \cdot)_{\mathcal{D}}$ to denote the $L^{2}$ scalar product on a domain $\mathcal{D}$, we use standard notations found in many textbooks on functional analysis. In what follows, we use the trace of $u_{i}$ and $u_{e}$ on the boundary. Therefore to work in the adequate mathematical framework we introduce the Hilbert (trace) space $H^{1 / 2}\left(\partial \Omega_{\alpha}\right)$ whose dual (the space of continuous linear functionals) is $H^{-1 / 2}\left(\partial \Omega_{\alpha}\right)$. Using the fact that the boundary $\Gamma_{m}$ is a subdomain of $\partial \Omega_{\alpha}$ and following the notation of [38] (Chapter 3), we introduce the Hilbert space

$$
H^{1 / 2}\left(\Gamma_{m}\right)=\left\{\left.u\right|_{\Gamma_{m}}, u \in H^{1 / 2}\left(\partial \Omega_{i}\right)\right\}=\left\{\left.u\right|_{\Gamma_{m}}, u \in H^{1 / 2}\left(\partial \Omega_{e}\right)\right\} .
$$

Note that the two definitions of $H^{1 / 2}\left(\Gamma_{m}\right)$ coincide since there exists a continuous extension operator from $H^{1 / 2}\left(\Gamma_{m}\right)$ to $H^{1 / 2}\left(\partial \Omega_{\alpha}\right)$ (see the proof of Theorem 4.10 in [38]). We denote by $\widetilde{H}^{-1 / 2}\left(\Gamma_{m}\right)$ the dual space and the duality pairing is denoted $\langle\cdot, \cdot\rangle_{\Gamma_{m}}$. For each $j \in$ $\widetilde{H}^{-1 / 2}\left(\Gamma_{m}\right)$, the standard dual norm is defined by

$$
\|j\|_{\widetilde{H}^{-1 / 2}\left(\Gamma_{m}\right)}=\sup _{0 \neq v \in H^{1 / 2}\left(\Gamma_{m}\right)} \frac{\left|\langle j, v\rangle_{\Gamma_{m}}\right|}{\|v\|_{H^{1 / 2}\left(\Gamma_{m}\right)}} .
$$

It is standard to assume that some positivity and symmetry properties are satisfied by the parameters of the system.

Assumption 1. The capacitance satisfies $C_{m}>0$ and the diffusion tensors $\vec{\sigma}_{\alpha}$ belong to $\left[L^{\infty}\left(\Omega_{\alpha}\right)\right]^{3 \times 3}$ and are symmetric, definite, positive and coercive, i.e. there exists $C>0$ such that

$$
\left(\overrightarrow{\vec{\sigma}}_{\alpha} \vec{\rho}, \vec{\rho}\right)_{\Omega_{\alpha}} \geq C\|\vec{\rho}\|_{L^{2}\left(\Omega_{\alpha}\right)}^{2}
$$

for all $\vec{\rho} \in L^{2}\left(\Omega_{\alpha}\right)^{3}$. This implies that

$$
\|\cdot\|_{L_{\sigma_{\alpha}}^{2}}:\left\{\begin{array}{ccc}
L^{2}\left(\Omega_{\alpha}\right) & \rightarrow & \mathbb{R}^{+} \\
\vec{\rho} & \mapsto & \sqrt{\left(\vec{\sigma}_{\alpha} \vec{\rho}, \vec{\rho}\right)_{\Omega_{\alpha}}}
\end{array}\right.
$$

defines a norm in $L^{2}\left(\Omega_{\alpha}\right)$. 


\subsection{Elimination of the quasi-static potential unknown}

Following an idea developed in [11] for the bidomain equation at the macroscopic level or in [29] for an electroporation model at the microscopic level, we rewrite System (11) by eliminating the unknown electric potentials $u_{\alpha}$ in $\Omega_{\alpha}$ and writing an equation for $V_{m}=$ $u_{i}-u_{e}$ on $\Gamma_{m}$. Note that the equation for the gating variable $w$ is kept because the only electric quantity involved is $V_{m}$ along $\Gamma_{m}$. We introduce the linears operators $\mathcal{T}_{i}$ and $\mathcal{T}_{e}$ that solve interior Laplace equations in $\Omega_{i}$ and $\Omega_{e}$ respectively. First, we define

$$
\mathcal{T}_{i}: H^{1 / 2}\left(\Gamma_{m}\right) \rightarrow \widetilde{H}^{-1 / 2}\left(\Gamma_{m}\right)
$$

which is given formally by $\mathcal{T}_{i}(v):=\overrightarrow{\vec{\sigma}}_{i} \vec{\nabla}_{\vec{x}} v_{i} \cdot \vec{n}_{i}$ along $\Gamma_{m}$, where $v_{i}$ is the unique solution of

$$
\left\{\begin{array}{rlll}
\vec{\nabla}_{\vec{x}} \cdot\left(\overrightarrow{\vec{\sigma}}_{i} \vec{\nabla}_{\vec{x}} v_{i}\right) & =0 & \Omega_{i}, \\
v_{i} & = & v & \Gamma_{m}, \\
\overrightarrow{\vec{\sigma}}_{i} \vec{\nabla}_{\vec{x}} v_{i} \cdot \vec{n}_{i} & = & 0 & \partial \Omega \cap \partial \Omega_{i} .
\end{array}\right.
$$

Since the problem above is well-posed (it is elliptic and coercive because $\Gamma_{m} \neq \emptyset$, see Theorem 4.10 of [38]), the linear functional $\mathcal{T}_{i}(v)$ can be rigorously defined by, for all $w \in$ $H^{1 / 2}\left(\Gamma_{m}\right)$,

$$
\left\langle\mathcal{T}_{i}(v), w\right\rangle_{\Gamma_{m}}=\left(\overrightarrow{\vec{\sigma}}_{i} \vec{\nabla}_{\vec{x}} v_{i}, \vec{\nabla}_{\vec{x}} w_{i}\right)_{\Omega_{i}},
$$

where $v_{i}$ is given by (12) and $w_{i} \in H^{1}\left(\Omega_{i}\right)$ is the unique solution of

$$
\left\{\begin{array}{rlll}
\vec{\nabla}_{\vec{x}} \cdot\left(\overrightarrow{\vec{\sigma}}_{i} \vec{\nabla}_{\vec{x}} w_{i}\right) & =0 & \Omega_{i} \\
w_{i} & = & w & \Gamma_{m} \\
\overrightarrow{\vec{\sigma}}_{i} \vec{\nabla}_{\vec{x}} w_{i} \cdot \vec{n}_{i} & = & 0 & \partial \Omega \cap \partial \Omega_{i} .
\end{array}\right.
$$

The operator $\mathcal{T}_{i}$ satisfies the properties summed up in the following proposition.

Proposition 1. If Assumption 1 holds, we have for all $(v, w) \in\left[H^{1 / 2}\left(\Gamma_{m}\right)\right]^{2}$,

$$
\begin{aligned}
& \left\langle\mathcal{T}_{i}(v), w\right\rangle_{\Gamma_{m}}=\left\langle\mathcal{T}_{i}(w), v\right\rangle_{\Gamma_{m}}, \\
& \left\langle\mathcal{T}_{i}(v), v\right\rangle_{\Gamma_{m}}=\left(\overrightarrow{\vec{\sigma}}_{i} \vec{\nabla}_{\vec{x}} v_{i}, \vec{\nabla}_{\vec{x}} v_{i}\right)_{\Omega_{i}} \geq 0, \\
& \left\|\mathcal{T}_{i}(v)\right\|_{H^{-1 / 2}\left(\Gamma_{m}\right)} \leq C\|v\|_{H^{1 / 2}\left(\Gamma_{m}\right)},
\end{aligned}
$$

where $C$ is a positive scalar depending only on $\overrightarrow{\vec{\sigma}}_{i}$ and the geometry.

Proof. By the definition of $\mathcal{T}_{i}$ and $v_{i}$, we have

$$
\left\langle\mathcal{T}_{i}(v), w\right\rangle_{\Gamma_{m}}=\left(\overrightarrow{\vec{\sigma}}_{i} \vec{\nabla}_{\vec{x}} v_{i}, \vec{\nabla}_{\vec{x}} w_{i}\right)_{\Omega_{i}}
$$

Moreover from the weak form of Problem (13), one can deduce that

$$
\left(\overrightarrow{\vec{\sigma}}_{i} \vec{\nabla}_{\vec{x}} w_{i}, \vec{\nabla}_{\vec{x}} v_{i}\right)_{\Omega_{i}}=\left\langle\mathcal{T}_{i}(w), v\right\rangle_{\Gamma_{m}}
$$

hence the first relation of the proposition. The second relation is obtained by setting $w=v$ in the previous equation and using the fact that $\overrightarrow{\vec{\sigma}}_{i}$ is a definite positive tensor (Assumption 1). Moreover, since $\overrightarrow{\vec{\sigma}}_{i}$ is $L^{\infty}$ (Assumption 1), we also have

$$
\sup _{w \neq 0} \frac{\left|\left\langle\mathcal{T}_{i}(v), w\right\rangle_{\Gamma_{m}}\right|}{\|w\|_{H^{1 / 2}\left(\Gamma_{m}\right)}} \leq C \frac{\left\|v_{i}\right\|_{H^{1}\left(\Omega_{i}\right)}\left\|w_{i}\right\|_{H^{1}\left(\Omega_{i}\right)}}{\|w\|_{H^{1 / 2}\left(\Gamma_{m}\right)}} .
$$

The third relation is then a consequence of stability results on elliptic problems with mixed boundary conditions (again see Theorem 4.10 of [38])) that map boundary data $v$ and $w$ to, respectively $v_{i}$ and $w_{i}$, i.e.

$$
\left\|v_{i}\right\|_{H^{1}\left(\Omega_{i}\right)} \leq C\|v\|_{H^{1 / 2}\left(\Gamma_{m}\right)} .
$$


Corollary 1. There exists $c>0$ such that for all $v \in H^{1 / 2}\left(\Gamma_{m}\right)$, we have

$$
\left\langle\mathcal{T}_{i}(v), v\right\rangle_{\Gamma_{m}}+\left|\int_{\Gamma_{m}} v d \gamma\right|^{2} \geq c\|v\|_{H^{1 / 2}\left(\Gamma_{m}\right)}^{2} .
$$

Proof. This result is obtained using the second relation of Proposition 1 and a Poincaré Wirtinger type inequality.

The operator $\mathcal{T}_{i}$ is used in order to substitute the first equation with $\alpha=i$ of System (11) into the third equation of the same system. This is possible since $u_{i}$ satisfies a static equation inside $\Omega_{i}$. The same argument holds for the extra-cellular potential $u_{e}$, therefore, for the same reason we introduce the operator

$$
\mathcal{T}_{e}: \widetilde{H}^{-1 / 2}\left(\Gamma_{m}\right) \rightarrow H^{1 / 2}\left(\Gamma_{m}\right),
$$

which is defined by $\mathcal{T}_{e}(j):=v_{e}$ along $\Gamma_{m}$, where $v_{e} \in H^{1}\left(\Omega_{e}\right)$ is the unique solution of

$$
\left\{\begin{array}{rlll}
\vec{\nabla}_{\vec{x}} \cdot\left(\overrightarrow{\vec{\sigma}}_{e} \vec{\nabla}_{\vec{x}} v_{e}\right) & =0 & \Omega_{e}, \\
\overrightarrow{\vec{\sigma}}_{e} \vec{\nabla}_{\vec{x}} v_{e} \cdot \vec{n}_{i} & =j-\frac{\langle j, 1\rangle_{\Gamma_{m}}}{\left|\Gamma_{m}\right|} & \Gamma_{m}, \\
\overrightarrow{\vec{\sigma}}_{e} \vec{\nabla}_{\vec{x}} v_{e} \cdot \vec{n}_{e} & =0 & \partial \Omega \cap \partial \Omega_{e}, \\
\int_{\Gamma_{m}} v_{e} d \gamma & =0 . &
\end{array}\right.
$$

Similar to the operator $\mathcal{T}_{i}$, the operator $\mathcal{T}_{e}$ satisfies some properties which are summed up in the following proposition.

Proposition 2. If Assumption 1 holds, we have for all $(j, k) \in\left[\widetilde{H}^{-1 / 2}\left(\Gamma_{m}\right)\right]^{2}$

$$
\begin{aligned}
& \left\langle k, \mathcal{T}_{e}(j)\right\rangle_{\Gamma_{m}}=\left\langle j, \mathcal{T}_{e}(k)\right\rangle_{\Gamma_{m}}, \\
& \left\langle j, \mathcal{T}_{e}(j)\right\rangle_{\Gamma_{m}}=-\left(\overrightarrow{\vec{\sigma}}_{e} \vec{\nabla}_{\vec{x}} v_{e}, \vec{\nabla}_{\vec{x}} v_{e}\right)_{\Omega_{e}} \leq 0, \\
& \left\|\mathcal{T}_{e}(j)\right\|_{H^{1 / 2}\left(\Gamma_{m}\right)} \leq C\|j\|_{\widetilde{H}^{-1 / 2}\left(\Gamma_{m}\right)},
\end{aligned}
$$

where $C$ is a positive scalar depending only on $\overrightarrow{\vec{\sigma}}_{e}$ and the geometry.

Proof. For all $k \in \widetilde{H}^{-1 / 2}\left(\Gamma_{m}\right)$, we define $w_{e} \in H^{1}\left(\Omega_{e}\right)$ such that,

$$
\left\{\begin{array}{rlll}
\vec{\nabla}_{\vec{x}} \cdot\left(\overrightarrow{\vec{\sigma}}_{e} \vec{\nabla}_{\vec{x}} w_{e}\right) & =0 & \Omega_{e} \\
\vec{\sigma}_{e} \vec{\nabla}_{\vec{x}} w_{e} \cdot \vec{n}_{i} & =k-\frac{\langle k, 1\rangle_{\Gamma_{m}}}{\left|\Gamma_{m}\right|} & \Gamma_{m} \\
\overrightarrow{\vec{\sigma}}_{e} \vec{\nabla}_{\vec{x}} w_{e} \cdot \vec{n}_{e} & =0 & & \partial \Omega \cap \partial \Omega_{e} \\
\int_{\Gamma_{m}} w_{e} d \gamma & = & 0 &
\end{array}\right.
$$

By the definition of $v_{e}$ and $w_{e}$, we deduce the two following equalities

$$
\left(\overrightarrow{\vec{\sigma}}_{e} \vec{\nabla}_{\vec{x}} v_{e}, \vec{\nabla}_{\vec{x}} w_{e}\right)_{\Omega_{e}}=-\left\langle j-\frac{\langle j, 1\rangle_{\Gamma_{m}}}{\left|\Gamma_{m}\right|}, \mathcal{T}_{e}(k)\right\rangle_{\Gamma_{m}}
$$

and

$$
\left(\overrightarrow{\vec{\sigma}}_{e} \vec{\nabla}_{\vec{x}} w_{e}, \vec{\nabla}_{\vec{x}} v_{e}\right)_{\Omega_{e}}=-\left\langle k-\frac{\langle k, 1\rangle_{\Gamma_{m}}}{\left|\Gamma_{m}\right|}, \mathcal{T}_{e}(j)\right\rangle_{\Gamma_{m}} .
$$

The first relation of the proposition is obtained by noticing that

$$
\int_{\Gamma_{m}} \mathcal{T}_{e}(j) d \gamma=\int_{\Gamma_{m}} \mathcal{T}_{e}(k) d \gamma=0 \text { hence }\left\langle\frac{\langle j, 1\rangle_{\Gamma_{m}}}{\left|\Gamma_{m}\right|}, \mathcal{T}_{e}(k)\right\rangle_{\Gamma_{m}}=\left\langle\frac{\langle k, 1\rangle_{\Gamma_{m}}}{\left|\Gamma_{m}\right|}, \mathcal{T}_{e}(j)\right\rangle_{\Gamma_{m}}=0 .
$$


The second relation of the proposition is obtained by setting $k=j$ in (15) and using the fact that $\vec{\sigma}_{e}$ is a definite positive tensor. To prove the continuity we first notice that

$$
\left\|\vec{\nabla}_{\vec{x}} v_{e}\right\|_{L^{2}\left(\Omega_{e}\right)}^{2} \leq C\|j\|_{\widetilde{H}^{-1 / 2}\left(\Gamma_{m}\right)}\left\|\mathcal{T}_{e}(j)\right\|_{H^{1 / 2}\left(\Gamma_{m}\right)}=C\|j\|_{\widetilde{H}^{-1 / 2}\left(\Gamma_{m}\right)}\left\|v_{e}\right\|_{H^{1 / 2}\left(\Gamma_{m}\right)},
$$

since $\overrightarrow{\vec{\sigma}}_{e} \in\left[L^{\infty}\left(\Omega_{\alpha}\right)\right]^{3 \times 3}$ (Assumption 1). Using the continuity of the extension $H^{1 / 2}\left(\Gamma_{m}\right)$ into $H^{1 / 2}\left(\partial \Omega_{e}\right)$, the continuity of the trace operator and finally a Poincaré -Wirtinger type inequality, we can show that

$$
\left\|v_{e}\right\|_{H^{1 / 2}\left(\Gamma_{m}\right)} \leq C\left(\left\|\vec{\nabla}_{\vec{x}} v_{e}\right\|_{L^{2}\left(\Omega_{e}\right)}+\left|\frac{1}{\left|\Gamma_{m}\right|} \int_{\Gamma_{m}} v_{e} d \gamma\right|\right) .
$$

Therefore $\left\|v_{e}\right\|_{H^{1 / 2}\left(\Gamma_{m}\right)} \leq C\left\|\vec{\nabla}_{\vec{x}} v_{e}\right\|_{L^{2}\left(\Omega_{e}\right)}$, since $v_{e}$ has zero average along $\Gamma_{m}$ and we finally obtain the relation $\left\|v_{e}\right\|_{H^{1 / 2}\left(\Gamma_{m}\right)} \leq C\|j\|_{H^{-1 / 2}\left(\Gamma_{m}\right)}$, hence the third inequality of the proposition.

Remark that our choice of definitions of $\mathcal{T}_{i}$ and $\mathcal{T}_{e}$ implies that

$$
\int_{\Gamma_{m}} u_{e} d \gamma=0
$$

Other choices are possible to define $u_{e}$ uniquely but are arbitrary and correspond to a choice of convention. Assuming that it has regular enough solutions $u_{\alpha}(t) \in H^{1}\left(\Omega_{\alpha}\right)$, for almost all $t \in[0, T]$, System (11) is equivalent to

$$
\left\{\begin{aligned}
C_{m} \partial_{t} V_{m}+I_{\text {ion }}^{\text {tot }}\left(V_{m}, w\right) & =-\mathcal{T}_{i}\left(u_{i}\right) & \Gamma_{m} \\
u_{e} & =\mathcal{T}_{e}\left(\mathcal{T}_{i}\left(u_{i}\right)\right) & \Gamma_{m} \\
\partial_{t} w & =-g\left(V_{m}, w\right) & \Gamma_{m}
\end{aligned}\right.
$$

where by definition $V_{m}=u_{i}-u_{e}$. Using the second equation of (17), we obtain

$$
u_{i}-\mathcal{T}_{e}\left(\mathcal{T}_{i}\left(u_{i}\right)\right)=V_{m} \quad \Gamma_{m}
$$

We can prove (see Lemma 1) that the operator $\mathcal{T}:=\mathcal{I}-\mathcal{T}_{e} \mathcal{T}_{i}: H^{1 / 2}\left(\Gamma_{m}\right) \rightarrow H^{1 / 2}\left(\Gamma_{m}\right)$ has a bounded inverse $(\mathcal{I}$ stands for the identity operator) and we therefore introduce the operator

$$
\mathcal{A}:\left\{\begin{array}{ccc}
H^{1 / 2}\left(\Gamma_{m}\right) & \rightarrow & \widetilde{H}^{-1 / 2}\left(\Gamma_{m}\right) \\
v & \rightarrow & \mathcal{T}_{i}\left(\mathcal{I}-\mathcal{T}_{e} \mathcal{T}_{i}\right)^{-1} v
\end{array}\right.
$$

and substitute the term $u_{i}$ in (17). This implies that if Assumption 1 holds, solutions of (11) satisfy

$$
\left\{\begin{array}{rlr}
C_{m} \partial_{t} V_{m}+I_{i o n}^{t o t}\left(V_{m}, w\right) & =-\mathcal{A}\left(V_{m}\right) & \Gamma_{m}, \\
\partial_{t} w & =-g\left(V_{m}, w\right) & \Gamma_{m} .
\end{array}\right.
$$

The converse is true. Solutions of (19) can be used to recover solutions of (11) as shown in Section 3.4. The properties of the operator $\mathcal{A}$ are summed up in the following proposition.

Proposition 3. If Assumption 1 holds, the linear operator $\mathcal{A}$ satisfies for all $(v, \tilde{v}) \in$ $\left[H^{1 / 2}\left(\Gamma_{m}\right)\right]^{2}$,

$$
\langle\mathcal{A}(v), \tilde{v}\rangle_{\Gamma_{m}}=\langle\mathcal{A}(\tilde{v}), v\rangle_{\Gamma_{m}} \text { and }\langle\mathcal{A}(v), v\rangle_{\Gamma_{m}} \geq 0 .
$$

Moreover, there exist constants $c$ and $C$ such that, for all $(v, \tilde{v}) \in\left[H^{1 / 2}\left(\Gamma_{m}\right)\right]^{2}$,

$$
\left|\langle\mathcal{A}(v), \tilde{v}\rangle_{\Gamma_{m}}\right| \leq C\|v\|_{H^{1 / 2}\left(\Gamma_{m}\right)}\|\tilde{v}\|_{H^{1 / 2}\left(\Gamma_{m}\right)}
$$

and

$$
\langle\mathcal{A}(v), v\rangle_{\Gamma_{m}}+\left|\int_{\Gamma_{m}} v d \gamma\right|^{2} \geq c\|v\|_{H^{1 / 2}\left(\Gamma_{m}\right)}^{2}
$$


Proof. To simplify the proof, we define $\theta \in H^{1 / 2}\left(\Gamma_{m}\right)$ and $\tilde{\theta} \in H^{1 / 2}\left(\Gamma_{m}\right)$ as

$$
\left(\mathcal{I}-\mathcal{T}_{e} \mathcal{T}_{i}\right)(\theta):=v \text { and }\left(\mathcal{I}-\mathcal{T}_{e} \mathcal{T}_{i}\right)(\tilde{\theta}):=\tilde{v} .
$$

Using Propositions 1 and 2, we obtain

$$
\langle\mathcal{A}(v), \tilde{v}\rangle_{\Gamma_{m}}=\left\langle\mathcal{A}(v),\left(\mathcal{I}-\mathcal{T}_{e} \mathcal{T}_{i}\right)(\tilde{\theta})\right\rangle_{\Gamma_{m}}=\left\langle\left(\mathcal{I}-\mathcal{T}_{i} \mathcal{T}_{e}\right) \mathcal{A}(v), \tilde{\theta}\right\rangle_{\Gamma_{m}} .
$$

Since by definition $\mathcal{A}=\mathcal{T}_{i}\left(\mathcal{I}-\mathcal{T}_{e} \mathcal{T}_{i}\right)^{-1}$ and using $\left(\mathcal{I}-\mathcal{T}_{i} \mathcal{T}_{e}\right) \mathcal{T}_{i}=\mathcal{T}_{i}\left(\mathcal{I}-\mathcal{T}_{e} \mathcal{T}_{i}\right)$, we deduce

$$
\langle\mathcal{A}(v), \tilde{v}\rangle_{\Gamma_{m}}=\left\langle\mathcal{T}_{i}(v), \tilde{\theta}\right\rangle_{\Gamma_{m}}=\left\langle\mathcal{T}_{i}(\tilde{\theta}), v\right\rangle_{\Gamma_{m}} .
$$

The symmetry of $\langle\mathcal{A}(\cdot), \cdot\rangle$ is then a consequence of the definition of $\tilde{\theta}$ as we have $\mathcal{T}_{i}(\tilde{\theta})=\mathcal{A}(\tilde{v})$. From (22), we can also deduce the non-negativity of the bilinear form by choosing $\tilde{v} \equiv v$. We find that

$$
\langle\mathcal{A}(v), v\rangle_{\Gamma_{m}}=\left\langle\mathcal{T}_{i}(\theta),\left(\mathcal{I}-\mathcal{T}_{e} \mathcal{T}_{i}\right)(\theta)\right\rangle_{\Gamma_{m}},
$$

which is non-negative thanks to the non-negativity of $\mathcal{T}_{i}$ and the non-positivity of $\mathcal{T}_{e}$. The continuity (20) is a direct consequence of the third equation of Proposition 1 and Lemma 1 (i.e. $\mathcal{T}:=\mathcal{I}-\mathcal{T}_{e} \mathcal{T}_{i}$ has a bounded inverse). Now remark that by the definition of $\mathcal{T}_{e}$, we have, for all $j \in \widetilde{H}^{-1 / 2}\left(\Gamma_{m}\right)$,

$$
\int_{\Gamma_{m}} \mathcal{T}_{e}(j) d \gamma=0 \Rightarrow \int_{\Gamma_{m}} v d \gamma=\int_{\Gamma_{m}} \mathcal{T}(\theta) d \gamma=\int_{\Gamma_{m}} \theta d \gamma
$$

Using the equalities above and (23), we find

$$
\langle\mathcal{A}(v), v\rangle_{\Gamma_{m}}+\left|\int_{\Gamma_{m}} v d \gamma\right|^{2} \geq\left\langle\mathcal{T}_{i}(\theta), \theta\right\rangle_{\Gamma_{m}}+\left|\int_{\Gamma_{m}} \theta d \gamma\right|^{2} .
$$

Corollary 1 shows the existence if of a positive scalar $c$ such that, for all $v \in H^{1 / 2}\left(\Gamma_{m}\right)$,

$$
\langle\mathcal{A}(v), v\rangle_{\Gamma_{m}}+\left|\int_{\Gamma_{m}} v d \gamma\right|^{2} \geq c\|\theta\|_{H^{1 / 2}\left(\Gamma_{m}\right)}=c\left\|\mathcal{T}^{-1}(v)\right\|_{H^{1 / 2}\left(\Gamma_{m}\right)} .
$$

Inequality (21) is then a consequence of the boundedness of $\mathcal{T}$ given by Lemma 1.

The last step of this section consists in proving the technical lemma regarding the invertibility of the operator $\mathcal{I}-\mathcal{T}_{e} \mathcal{T}_{i}$, which allows to define the operator $\mathcal{A}$.

Lemma 1. The linear operator

$$
\mathcal{T}:=\mathcal{I}-\mathcal{T}_{e} \mathcal{T}_{i}: H^{1 / 2}\left(\Gamma_{m}\right) \rightarrow H^{1 / 2}\left(\Gamma_{m}\right)
$$

is bounded and has a bounded inverse.

Proof. Using Propositions 1 and 2, we see that the operator $\mathcal{T}$ is linear and bounded, and hence continuous. In what follows, we prove that $\mathcal{T}$ is injective and then we deduce a lower bound for the norm of $\mathcal{T}$. Finally, we prove that the range of the operator is closed and that its orthogonal is the null space. These two last steps allow to show that the range of $\mathcal{T}$ is $H^{1 / 2}\left(\Gamma_{m}\right)$ and the result follows from the bounded inverse theorem.

\section{Step 1: Injectivity of the operator}

For any $v \in H^{1 / 2}\left(\Gamma_{m}\right)$ such that $\mathcal{T}(v)=0$, we have $\left\langle\mathcal{T}_{i}(v), v\right\rangle_{\Gamma_{m}}=\left\langle\mathcal{T}_{i}(v), \mathcal{T}_{e} \mathcal{T}_{i}(v)\right\rangle_{\Gamma_{m}}$. The first term of the equality is non-negative (Proposition 1 ) while the second is non-positive thanks to Proposition 2. Therefore, we obtain $\left\langle\mathcal{T}_{i}(v), v\right\rangle_{\Gamma_{m}}=0$ and this implies thanks to Proposition 1 that $v$ is constant along $\Gamma_{m}$. However, for any constant function $c$, we have 
$\mathcal{T}_{i}(c)=0$ therefore, in our case, $\mathcal{T}(v)=0$ implies $v=\mathcal{T}_{e} \mathcal{T}_{i}(v)=0$.

Step 2: Lower bound for the operator norm

For all $\theta \in H^{1 / 2}\left(\Gamma_{m}\right)$, we define $v \in H^{1 / 2}\left(\Gamma_{m}\right)$ as $\mathcal{T}(\theta)=v$. Then, as written in Equation (24), $\theta$ and $v$ have the same average along $\Gamma_{m}$ and

$$
\mathcal{T}\left(\theta_{0}\right)=v_{0} \text { where } \theta_{0}:=\theta-\frac{1}{\left|\Gamma_{m}\right|} \int_{\Gamma_{m}} \theta d \gamma \text { and } v_{0}:=v-\frac{1}{\left|\Gamma_{m}\right|} \int_{\Gamma_{m}} v d \gamma
$$

We have

$$
\left\langle\mathcal{T}_{i}\left(\theta_{0}\right), v_{0}\right\rangle_{\Gamma_{m}}=\left\langle\mathcal{T}_{i}\left(\theta_{0}\right), \mathcal{T}\left(\theta_{0}\right)\right\rangle_{\Gamma_{m}}=\left\langle\mathcal{T}_{i}\left(\theta_{0}\right), \theta_{0}\right\rangle_{\Gamma_{m}}-\left\langle\mathcal{T}_{i}\left(\theta_{0}\right), \mathcal{T}_{e} \mathcal{T}_{i}\left(\theta_{0}\right)\right\rangle_{\Gamma_{m}}
$$

Since $\mathcal{T}_{i}$ is non negative and $\mathcal{T}_{e}$ non positive (Proposition 1 and 2), we deduce

$$
\left\langle\mathcal{T}_{i}\left(\theta_{0}\right), \theta_{0}\right\rangle \leq\left\|\mathcal{T}_{i}\left(\theta_{0}\right)\right\|_{\widetilde{H}^{-1 / 2}\left(\Gamma_{m}\right)}\left\|v_{0}\right\|_{H^{1 / 2}\left(\Gamma_{m}\right)} \leq C\left\|\theta_{0}\right\|_{H^{1 / 2}\left(\Gamma_{m}\right)}\left\|v_{0}\right\|_{H^{1 / 2}\left(\Gamma_{m}\right)},
$$

where $C$ is the continuity constant given by Proposition 1 . Using Corollary 1 and the fact that $\theta_{0}$ has zero average along $\Gamma_{m}$, we have $c\left\|\theta_{0}\right\|_{H^{1 / 2}\left(\Gamma_{m}\right)}^{2} \leq\left\langle\mathcal{T}_{i}\left(\theta_{0}\right), \theta_{0}\right\rangle$, therefore using (25), we find

$$
\left\|\theta_{0}\right\|_{H^{1 / 2}\left(\Gamma_{m}\right)} \leq \frac{C}{c}\left\|v_{0}\right\|_{H^{1 / 2}\left(\Gamma_{m}\right)} .
$$

Finally, we get

$$
\begin{aligned}
\|\theta\|_{H^{1 / 2}\left(\Gamma_{m}\right)} \leq\left\|\theta_{0}\right\|_{H^{1 / 2}\left(\Gamma_{m}\right)}+\mid \frac{1}{\left|\Gamma_{m}\right|} & \int_{\Gamma_{m}} \theta d \gamma \mid\|1\|_{H^{1 / 2}\left(\Gamma_{m}\right)} \\
& \leq \frac{C}{c}\left\|v_{0}\right\|_{H^{1 / 2}\left(\Gamma_{m}\right)}+\left|\frac{1}{\left|\Gamma_{m}\right|} \int_{\Gamma_{m}} v d \gamma\right|\|1\|_{H^{1 / 2}\left(\Gamma_{m}\right)} .
\end{aligned}
$$

This implies that there exists another constant $C$ depending only on the geometry and $\overrightarrow{\vec{\sigma}}_{i}$ such that $\|\theta\|_{H^{1 / 2}\left(\Gamma_{m}\right)} \leq C\|v\|_{H^{1 / 2}\left(\Gamma_{m}\right)}=C\|\mathcal{T}(\theta)\|_{H^{1 / 2}\left(\Gamma_{m}\right)}$.

Step 3: Orthogonal of the operator range

Let $j \in \widetilde{H}^{-1 / 2}\left(\Gamma_{m}\right)$ such that for all $v \in H^{1 / 2}\left(\Gamma_{m}\right)$,

$$
\langle j, \mathcal{T}(v)\rangle_{\Gamma_{m}}=0 .
$$

We choose $v=\mathcal{T}_{e}(j)$ in (26) and thanks to Propositions 1 and 2, we find

$$
\left\langle j, \mathcal{T}_{e}(j)\right\rangle_{\Gamma_{m}}=\left\langle j, \mathcal{T}_{e} \mathcal{T}_{i} \mathcal{T}_{e}(j)\right\rangle_{\Gamma_{m}}=\left\langle\mathcal{T}_{i} \mathcal{T}_{e}(j), \mathcal{T}_{e}(j)\right\rangle_{\Gamma_{m}}
$$

The last term is non negative therefore, since $\left\langle\mathcal{T}_{e}(j), j\right\rangle_{\Gamma_{m}}$ is non positive, it should vanish. This implies that $j$ is constant along $\Gamma_{m}$ (since in (14), $\left.\vec{\nabla}_{\vec{x}} v_{e}=0\right)$. Now, we choose $v \equiv 1$ in (26) and we find

$$
\langle j, \mathcal{T}(v)\rangle_{\Gamma_{m}}=0 \Rightarrow\langle j, 1\rangle_{\Gamma_{m}}=0 \Rightarrow j=0 .
$$

We are now ready to give the required assumptions to prove the existence and the uniqueness of System (19). 


\subsection{Mathematical assumptions for the well-posedness}

As mentioned in [63], no maximum principle has been proven for Problem (19) (or any linearized version) contrary to standard reaction-diffusion problems. The consequence is that one can not deduce easily bounds in time and space for the electric variable $V_{m}$. This implies that some specific assumptions are required to guarantee that non-linear terms involving $V_{m}$ are well-defined and that existence results hold. More generally, in this section, the required assumptions to prove existence and/or uniqueness of weak solutions of System (19) are presented.

First, from the equivalence of the bidomain equations and System (19) (see Section 3.1), it is clear that our problem has to be completed with the following initial conditions on $\Gamma_{m}$

$$
\left\{\begin{aligned}
V_{m}(\cdot, 0) & =u_{i}(\cdot, 0)-u_{e}(\cdot, 0)=V_{m}^{0} \\
w(\cdot, 0) & =w^{0}
\end{aligned}\right.
$$

It is sufficient to require the following assumption concerning the initial conditions.

Assumption 2. Properties of the initial conditions.

$$
V_{m}^{0} \in L^{2}\left(\Gamma_{m}\right) \quad \text { and } \quad w^{0} \in L^{2}\left(\Gamma_{m}\right)
$$

As already said, the ionic term $I_{i o n}^{t o t}$ is decomposed into two parts

$$
I_{\text {ion }}^{t o t}=I_{i o n}-I_{a p p},
$$

where $I_{a p p}$ is the applied current and is a function of time and space. We assume the following property concerning the applied current.

\section{Assumption 3. Property of the source term.}

$$
I_{a p p} \in L^{2}\left(\Gamma_{m} \times(0, T)\right) .
$$

To represent the variety of the behavior of the cardiac cells, we mathematically define the ionic terms $I_{i o n}$ and $g$ by introducing a family of functions on $\mathbb{R}^{2}$ parametrized by the space variable

$$
I_{\text {ion }}(\vec{x}, \cdot): \mathbb{R}^{2} \longrightarrow \mathbb{R} \text { and } g(\vec{x}, \cdot): \mathbb{R}^{2} \longrightarrow \mathbb{R} .
$$

At each fixed $\vec{x} \in \Omega$, the ionic terms $I_{\text {ion }}$ and $g$ describe a different behavior that corresponds to a non-linear reaction term. Therefore to further the mathematical analysis, it is assumed that these functions have some regularity in all their arguments. This leads to the following assumption.

\section{Assumption 4. Regularity condition.}

$$
I_{\text {ion }} \in C^{0}\left(\bar{\Omega} \times \mathbb{R}^{2}\right) \quad \text { and } \quad g \in C^{0}\left(\bar{\Omega} \times \mathbb{R}^{2}\right) .
$$

In the mathematical analysis of macroscopic bidomain equations, several paths have been followed in the literature according to the definition of the ionic current. We summarize below the encountered various cases.

\section{Physiological models}

For these models, one can prove that the gating variable $w$ is bounded from below and above, due to the specific structure of (6) and (7). To go further in the physiological description, some models consider the concentrations as variables of the system, see for example the Luo-Rudy model [35]. In [63, 64], such models are considered.

\section{Phenomenological models}

a) The FitzHugh like models (8)

The FitzHugh like models have been studied in $[17,10,11,16]$. In these models, there are no obvious bounds on the gating variable. The FitzHugh-Nagumo model satisfies some 
good mathematical properties (existence and uniqueness of solutions for arbitrary observation times) whereas the Aliev-Panfilov and MacCulloch model still rise some mathematical difficulties. In particular, no proof of uniqueness of solutions exists in the literature because of the non-linearity in the coupling terms between $w$ and $V_{m}$.

b) The Mitchell-Schaeffer model (9)

The Mitchell-Schaeffer model has been studied in [10, 32, 16]. This model and its regularized version have a very specific structure. First, we will show that the gating variable is bounded from below and above but uniqueness of the solution is a difficult mathematical question which is addressed in [32] for a related ordinary differential (ODE) problem.

In what follows, we describe in detail the structures of $I_{i o n}$ and $g$. In all models, some "growth conditions" are required to write the problem in an adequate variational framework.

Assumption 5. Growth condition. There exists a scalar $C_{\infty}>0$ such that for all $\vec{x} \in \Omega$ and $(v, w) \in \mathbb{R}^{2}$ we have

$$
\left|I_{\text {ion }}(\vec{x}, v, w)\right| \leq C_{\infty}\left(|v|^{3}+|w|+1\right)
$$

and

$$
|g(\vec{x}, v, w)| \leq C_{\infty}\left(|v|^{2}+|w|+1\right) .
$$

Remark 2. The inclusion $H^{1 / 2}\left(\Gamma_{m}\right)$ into $L^{4}\left(\Gamma_{m}\right)$ is continuous, see Proposition 2.4 of [17], therefore by identification of integrable functions with linear forms, there is a continuous inclusion of $L^{4 / 3}\left(\Gamma_{m}\right)$ into $\widetilde{H}^{-1 / 2}\left(\Gamma_{m}\right)$. Moreover if

$$
v \in L^{2}\left((0, T), H^{1 / 2}\left(\Gamma_{m}\right)\right), \quad w \in L^{2}\left((0, T) \times \Gamma_{m}\right),
$$

we have (the dependency w.r.t. $\vec{x}$ is omitted for the sake of clarity)

$$
I_{\text {ion }}(v, w) \in L^{2}\left((0, T), L^{4 / 3}\left(\Gamma_{m}\right)\right) \quad \text { and } \quad g(v, w) \in L^{2}\left((0, T) \times \Gamma_{m}\right) .
$$

Remark 3. Several mathematical analyses concern only the macroscopic bidomain equations (see [11, 10, 8] for instance) and these analyses can be extended to the case of the microscopic bidomain equations with some slightly different assumptions on the non-linear terms $I_{i o n}$ and $\mathrm{g}$. These assumptions take into account the functional framework in which we have to work. Namely, we have to use the trace space $H^{1 / 2}\left(\Gamma_{m}\right)$ instead of the more standard functional space $H^{1}(\Omega)$. More precisely in [11], the growth conditions are

$$
\left|I_{\text {ion }}(\vec{x}, v, w)\right| \leq C_{\infty}\left(|v|^{5}+|w|+1\right)
$$

and

$$
|g(\vec{x}, v, w)| \leq C_{\infty}\left(|v|^{3}+|w|+1\right) .
$$

In that case, it can be shown that $I_{i o n}(v, w)$ and $g(v, w)$ are integrable and well defined if $v \in H^{1}(\Omega)$ and $w \in L^{2}(\Omega)$.

As mentioned in [17], the growth conditions (28) and (29) are there to ensure that the functions $I_{i o n}$ and $g$ can be used to construct models that are well defined in the variational sense. The growth condition (Assumption 5) is not sufficient to guaranty the existence of solutions of Problem (19). Indeed, since $I_{i o n}$ can behave like a cubic polynomial for large value of $v$ and the function $g$ could behave as a quadratic polynomial in $v$, it turns out to be necessary to have a signed condition (see Equations (51) - (54) for more insight). This leads to the following assumption

Assumption 6. There exist $\mu>0$ and $C_{I}>0$ such that for all $\vec{x} \in \Omega$ and $(v, w) \in \mathbb{R}^{2}$, we have

$$
v I_{i o n}(\vec{x}, v, w)+\mu w g(\vec{x}, v, w) \geq-C_{I}\left(|v|^{2}+|w|^{2}+1\right) .
$$


In general, $V_{m}$ is more regular in space than $w$ due to the presence of the positive operator $\mathcal{A}$. Due to this lack of regularity on the gating variables, some additional assumptions have to hold to carry out the mathematical analysis. Concerning this question two kinds of assumption are proposed in the literature. Roughly speaking, these assumptions depend on if the model is physiological or phenomenological. To be able to refine our analysis depending on the different properties of the models, we make the following assumption.

Assumption 7. One of the following assumptions hold

a) Global lipschitz property. There exists a positive scalar $L_{g}>0$, such that for all $\vec{x} \in \Omega,\left(v_{1}, v_{2}\right) \in \mathbb{R}^{2}$ and $\left(w_{1}, w_{2}\right) \in \mathbb{R}^{2}$,

$$
\left|g\left(\vec{x}, v_{1}, w_{1}\right)-g\left(\vec{x}, v_{2}, w_{2}\right)\right| \leq L_{g}\left|v_{1}-v_{2}\right|+L_{g}\left|w_{1}-w_{2}\right| .
$$

b) Decomposition of the non-linear terms. There exist continuous functions

$$
\left(f_{1}, f_{2}, g_{1}, g_{2}\right) \in\left[C^{0}\left(\Omega \times \mathbb{R}^{2}\right)\right]^{4}
$$

such that

$$
\left\{\begin{array}{l}
I_{\text {ion }}(\vec{x}, v, w)=f_{1}(\vec{x}, v)+f_{2}(\vec{x}, v) w, \\
g(\vec{x}, v, w)=g_{1}(\vec{x}, v)+g_{2}(\vec{x}) w,
\end{array}\right.
$$

and there exist positive constants $C_{1}, c_{1}$ and $C_{2}$ such that for all $\vec{x} \in \Omega$ and $v \in \mathbb{R}$,

$$
v f_{1}(\vec{x}, v) \geq C_{1}|v|^{4}-c_{1}\left(|v|^{2}+1\right) \quad \text { and } \quad\left|f_{2}(\vec{x}, v)\right| \leq C_{2}(|v|+1) .
$$

Remark 4. The existence result of solutions given later (Theorem 1) is valid with either Assumption 7a or 7b. Note also that Assumption 7a is satisfied for the physiological models, the Mitchell-Schaeffer model and the FitzHugh-Nagumo model (the simplest form of FitzHugh-like models), whereas Assumption $7 b$ holds for the Aliev-Panfilov and MacCulloch models.

Finally, to prove the uniqueness of a solution for the microscopic bidomain problem, the terms $I_{i o n}$ and $g$ have to satisfy a global signed Lipschitz relation (the following assumption is a variant of what is suggested in [17] or [11] and [10]).

Assumption 8. One-sided Lipschitz condition. There exist $\mu>0$ and $L_{I}>0$ such that for all $\vec{x} \in \Omega,\left(v_{1}, w_{1}\right) \in \mathbb{R}^{2}$ and $\left(v_{2}, w_{2}\right) \in \mathbb{R}^{2}$

$$
\begin{array}{r}
\left(I_{\text {ion }}\left(\vec{x}, v_{1}, w_{1}\right)-I_{i o n}\left(\vec{x}, v_{2}, w_{2}\right)\right)\left(v_{1}-v_{2}\right)+\mu\left(g\left(\vec{x}, v_{1}, w_{1}\right)-g\left(\vec{x}, v_{2}, w_{2}\right)\right)\left(w_{1}-w_{2}\right) \\
\geq-L_{I}\left(\left|v_{1}-v_{2}\right|^{2}+\left|w_{1}-w_{2}\right|^{2}\right) .
\end{array}
$$

Note that such an assumption is not satisfied for the Aliev-Panfilov, the MacCulloch and the Mitchell-Schaeffer models. Uniqueness of a solution for these models is still an open problem.

For physiological models and for the Mitchell-Schaeffer model, there exist two finite scalars $w_{\min }<w_{\max }$ such that we expect the solution $w$ to be bounded from below by $w_{\min }$ and above by $w_{\max }$. For this to be true, it has to be satisfied for the initial data and this leads to the following assumptions when considering such models.

Assumption 9.

$$
w_{\min } \leq w^{0}(\cdot) \leq w_{\max }, \quad \Gamma_{m}
$$

Assumption 10. For all $\vec{x} \in \Omega$ and $v \in \mathbb{R}$,

$$
g\left(\vec{x}, v, w_{\text {min }}\right) \leq 0 \text { and } g\left(\vec{x}, v, w_{\max }\right) \geq 0 .
$$


The last assumption is satisfied when considering the function $g$ defined as in physiological models $(5,6)$ or as in the Mitchell-Schaeffer model (10). For such models, the terms $I_{i o n}(\vec{x}, v, w)$ and $g(\vec{x}, v, w)$ should be replaced by $I_{\text {ion }}(\vec{x}, v, \chi(w))$ and $g(\vec{x}, v, \chi(w))$ respectively, where

$$
\chi(w)= \begin{cases}w_{\min } & w \leq w_{\min } \\ w_{\max } & w \geq w_{\max } \\ w & \text { otherwise }\end{cases}
$$

Note that with such substitutions, we do not modify the solution while the global conditions of Assumptions 5, 6, 7a and 8 are more likely to be fulfilled since it corresponds to verify local conditions on $w$. As an example, we can remark that the physiological models - of the form given by $(5,6)$ - do not satisfy the Lipschitz property (Assumption 8) globally in $w$ but only locally. However, for these models, we can show a priori that $w$ is bounded from below and above, hence the suggested modification of the non-linear terms. Finally, note that this assumption is also a physiological assumption. Indeed, it makes sense to have some bounds on the gating variables.

Remark 5. Assumptions 1-4 are always satisfied and do not depend on the structure of the non-linear terms $I_{i o n}$ and $g$. Moreover, it is possible to classify the models of the literature depending on which assumption they satisfy, see Table 5. We refer the reader to [11] for

\begin{tabular}{c|c|c|c|c|c|} 
Assumption & 5 & 6 & 7 & 8 & $9-10$ \\
\hline FitzHugh-Nagumo & $\checkmark$ & $\checkmark$ & $a)$ & $\checkmark$ & \\
Roger MacCulloch & $\checkmark$ & $\checkmark$ & $a)$ & & \\
Aliev-Panfilov & $\checkmark$ & $\checkmark$ & $b)$ & & \\
Regularized Mitchell-Schaeffer & $\checkmark$ & $\checkmark$ & $a)$ & & $\checkmark$ \\
Physiological models & $\checkmark$ & $\checkmark$ & $a)$ & $\checkmark$ & $\checkmark$ \\
\hline
\end{tabular}

Table 1: Ionic models and verified assumptions

the analysis of FitzHugh-Nagumo, Roger MacCulloch and Aliev-Panfilov assumptions and Section 2 for the regularized Mitchell-Schaeffer and physiological models.

\subsection{Existence and uniqueness analysis}

All the proofs of this section are given in Appendix 5.

In the literature, the analysis of the classical bidomain model is done most of the time at the macroscopic scale. Equations at that scale are obtained from the microscopic model using a formal asymptotic homogenization procedure (see [43, 17]) or the $\Gamma$-convergence method (see $[6,50])$. In any cases, to justify the homogenization process, a complete mathematical analysis at the microscopic scale is necessary. In this section, we give existence and uniqueness results for solutions of System (11). Note that the existence of solutions for the macroscopic bidomain equation used in the literature is a consequence of the 2-scale convergence theory presented in the next section.

In the literature, one can find three different approaches that are used for the mathematical analysis of the macroscopic classical bidomain equations. Following the classification suggested in the recent book [16], these three approaches are

1. The use of the abstract framework of degenerate evolution variational inequalities in Hilbert spaces (see for instance [17]). Such an approach has been used to do the analysis when FitzHugh-Nagumo models are considered and is adapted to the analysis of semi-discretization in time of the problem (see [57]).

2. The use of Schauder fixed point theorem. This is the approach suggested in [63] and [64]. In these references, the ionic term depends on the concentration of ionic species (in addition to the dependence on the gating variables). This approach is adapted to the analysis of physiological models. 
3. The Faedo-Galerkin approach. This approach is used in the context of electrophysiology in $[8,10,11]$ and in the context of electroporation in [29]. It is the most versatile approach although it has been used to analyze only phenomenological models in the mentioned references. We refer the reader to the textbook of J.-L. Lions [33] for a detailed description. This technique is based upon a limit process of discretization in space of the partial differential equations combined with the use of standard results on systems of ODEs (at each fixed discretization). This is the approach that we consider in the appendix of this paper.

In what follows, we proceed in three steps. The first step consists in showing existence/uniqueness results for the evolution equation of the gating variable $w$. More precisely, given any (electric potential)

$$
U \in C^{0}\left([0, T] ; L^{2}\left(\Gamma_{m}\right)\right),
$$

we show in the appendix that the associated gating variable - solution of (35) - is bounded from below and above when physiological or Mitchell-Schaeffer models are concerned. The next step concerns existence results for the full non-linear microscopic bidomain equations and the final step gives the uniqueness result.

\section{Step 1 - Evolution equation of the gating variable.}

The term $g\left(V_{m}, w\right)$ in $(19)$ is replaced by the term $g(U, w)$ and we denote the corresponding solution $w=w_{U}$. As mentioned previously, our main purpose here is to state the fact that - in the case of physiological or Mitchell-Schaeffer models - the solution $w_{U}$ is bounded from below and above.

Lemma 2. If Assumption 2, 4, 5 and 7a hold, there exists a unique function

$$
w_{U} \in C^{1}\left([0, T] ; L^{2}\left(\Gamma_{m}\right)\right)
$$

which is a solution of

$$
\begin{cases}\partial_{t} w_{U}+g\left(\vec{x}, U, w_{U}\right)=0, & \Gamma_{m}, \forall t \in[0, T] \\ w_{U}(\vec{x}, 0)=w^{0}(\vec{x}), & \Gamma_{m}\end{cases}
$$

Moreover if Assumptions 9 and 10 are satisfied then for all $t \in[0, T]$ and almost all $\vec{x} \in \Gamma_{m}$,

$$
w_{\min } \leq w_{U}(\vec{x}, t) \leq w_{\max } .
$$

The proof of Lemma 2 is done by considering smooth approximations of $U$ and $w^{0}$. Then, the problem reduces to the analysis of an ordinary differential equation where the space variable $\vec{x}$ plays the role of a parameter. Finally, the solution of (35) is constructed by a limit process using the density of smooth functions into $L^{2}\left(\Gamma_{m}\right)$.

\section{Step 2 - Existence result for the microscopic bidomain equation}

Theorem 1. If Assumptions 1-7 hold, there exist

$$
V_{m} \in C^{0}\left([0, T] ; L^{2}\left(\Gamma_{m}\right)\right) \cap L^{2}\left((0, T) ; H^{1 / 2}\left(\Gamma_{m}\right)\right), \quad \partial_{t} V \in L^{2}\left((0, T) ; H^{-1 / 2}\left(\Gamma_{m}\right)\right),
$$

and

$$
w \in H^{1}\left((0, T) ; L^{2}\left(\Gamma_{m}\right)\right)
$$

which are solutions of

$$
\left\{\begin{array}{lll}
C_{m} \partial_{t} V_{m}+\mathcal{A}\left(V_{m}\right)+I_{i o n}\left(V_{m}, w\right)=I_{a p p}, & \widetilde{H}^{-1 / 2}\left(\Gamma_{m}\right), & \text { a.e. } t \in(0, T), \\
\partial_{t} w+g\left(V_{m}, w\right)=0, & \Gamma_{m}, & \text { a.e. } t \in(0, T)
\end{array}\right.
$$

and

$$
\begin{cases}V_{m}(\vec{x}, 0)=V_{m}^{0}(\vec{x}) & \Gamma_{m} \\ w(\vec{x}, 0)=w^{0}(\vec{x}) & \Gamma_{m}\end{cases}
$$


The proof of Theorem 1 is done using the Faedo-Galerkin method. More precisely, the equations are first space-discretized using a finite dimensional basis of $L^{2}\left(\Gamma_{m}\right)$ constructed with the eigenvectors of $\mathcal{A}$. After the discretization, it is proven that semi-discrete solutions exist by applying the Cauchy-Peano theorem (to be more specific, we use the more general Carathéodory's existence theorem) on systems of ordinary differential equations. Finally, by a limit procedure, the existence of solutions is proven for the weak form of (36) (the limit procedure uses compactness results to deduce strong convergence of the semi-discrete solutions. This strategy allows to pass to the limit in the non-linear terms $I_{i o n}$ and $g$ ).

Remark 6. If Assumption Ya is valid (i.e. $g$ is globally Lipschitz) then by application of Lemma 2 the solution given by Theorem 1 has the additionnal regularity

$$
w \in C^{1}\left([0, T] ; L^{2}\left(\Gamma_{m}\right)\right) .
$$

\section{Step 3 - Uniqueness results for the microscopic bidomain equation}

Uniqueness is proven by standard energy techniques for models satisfying the one sided Lipschitz property, see Assumption 8.

Corollary 2. If Assumption 8 holds, then the solution of the microscopic bidomain equations given by Theorem 1 is unique.

\subsection{Post-processing of the intra- and extra-cellular potentials}

From the solution $V_{m}=u_{i}-u_{e}$ given by Theorem 1, we can first recover the intra-cellular potential

$$
u_{i} \in L^{2}\left((0, T) ; H^{1}\left(\Omega_{i}\right)\right) .
$$

Using Equation (18), one can see that it is defined as the unique solution of the following quasi-static elliptic problem (the time-dependence appears only in the boundary data),

$$
\left\{\begin{array}{rlrl}
\vec{\nabla}_{\vec{x}} \cdot\left(\overrightarrow{\vec{\sigma}}_{i} \vec{\nabla}_{\vec{x}} u_{i}\right) & =0 & \Omega_{i}, \\
u_{i} & =\left(\mathcal{I}-\mathcal{T}_{e} \mathcal{T}_{i}\right)^{-1} V_{m} & \Gamma_{m}, \\
\overrightarrow{\vec{\sigma}}_{i} \vec{\nabla}_{\vec{x}} u_{i} \cdot \vec{n}_{i} & =0 & & \partial \Omega \cap \partial \Omega_{i} .
\end{array}\right.
$$

In the same way, the extra-cellular potential

$$
u_{e} \in L^{2}\left((0, T) ; H^{1}\left(\Omega_{e}\right)\right)
$$

is defined as the unique solution of the following quasi-static elliptic problem,

$$
\left\{\begin{array}{rll}
\vec{\nabla}_{\vec{x}} \cdot\left(\overrightarrow{\vec{\sigma}}_{e} \vec{\nabla}_{\vec{x}} u_{e}\right) & =0 & \Omega_{e} \\
\overrightarrow{\vec{\sigma}}_{e} \vec{\nabla}_{\vec{x}} u_{e} \cdot \vec{n}_{i} & =\mathcal{T}_{i}\left(u_{i}\right)-\frac{\left\langle\mathcal{T}_{i}\left(u_{i}\right), 1\right\rangle}{\left|\Gamma_{m}\right|} & \Gamma_{m} \\
\overrightarrow{\vec{\sigma}}_{e} \vec{\nabla}_{\vec{x}} u_{e} \cdot \vec{n}_{e} & =0 & \\
\int_{\Gamma_{m}} u_{e} d \gamma & =0 &
\end{array}\right.
$$

From the definition above, one can recover energy estimates on $\left(V_{m}, w, u_{i}, u_{e}\right)$ from the energy estimates derived for the system (36) where only $\left(V_{m}, w\right)$ appears. To do so, we will use later the following proposition.

Proposition 4. Let $V_{m} \in L^{2}\left((0, T) ; H^{1 / 2}\left(\Gamma_{m}\right)\right)$, then for almost all $t \in(0, T)$, we have

$$
\left\langle\mathcal{A}\left(V_{m}\right), V_{m}\right\rangle_{\Gamma_{m}}=\left(\overrightarrow{\vec{\sigma}}_{i} \vec{\nabla}_{\vec{x}} u_{i}, \vec{\nabla}_{\vec{x}} u_{i}\right)_{\Omega_{i}}+\left(\overrightarrow{\vec{\sigma}}_{e} \vec{\nabla}_{\vec{x}} u_{e}, \vec{\nabla}_{\vec{x}} u_{e}\right)_{\Omega_{e}}
$$

where $\left(u_{i}, u_{e}\right)$ are given by (38) and (39). 
Proof. We have

$$
\begin{aligned}
\left\langle\mathcal{A}\left(V_{m}\right), V_{m}\right\rangle_{\Gamma_{m}}=\left\langle\mathcal{T}_{i} \mathcal{T}^{-1}\left(V_{m}\right), V_{m}\right\rangle_{\Gamma_{m}}=\left\langle\mathcal{T}_{i} \mathcal{T}^{-1}\left(V_{m}\right), \mathcal{T} \mathcal{T}^{-1}\left(V_{m}\right)\right\rangle_{\Gamma_{m}} \\
=\left\langle\mathcal{T}_{i} \mathcal{T}^{-1}\left(V_{m}\right), \mathcal{T}^{-1}\left(V_{m}\right)\right\rangle_{\Gamma_{m}}-\left\langle\mathcal{T}_{i} \mathcal{T}^{-1}\left(V_{m}\right), \mathcal{T}_{e} \mathcal{T}_{i} \mathcal{T}^{-1}\left(V_{m}\right)\right\rangle_{\Gamma_{m}}
\end{aligned}
$$

The first term of the right-hand side gives by definition and Proposition 1 the quadratic term on $u_{i}$ whereas the second term gives by definition of $\mathcal{T}_{e}, u_{e}$ and Proposition 2 the quadratic term on $u_{e}$.

We are now in position to perform a rigorous homogenization of the microscopic bidomain model (2).

\section{Homogenization of the bidomain equations}

The microscopic model is unusable for the whole heart in term of numerical applications. At the macroscopic scale, the heart appears as a continuous material with a fiber-based structure. At this scale, the intracellular and extracellular media are undistinguishable. Our objective is to use a homogenization of the microscopic bidomain model in order to obtain a bidomain model where all the unknowns are defined everywhere hence simplifying the geometry of the domain. Formally after homogenization, we consider that the cardiac volume is " $\Omega=\Omega_{i}=\Omega_{e}$ ".

Homogenization of partial differential systems is a well known technique (see [9] for a reference textbook on the matter). It is done by considering the medium as periodic, with the period denoted by $\varepsilon$, then by constructing equations deduced by asymptotic analysis w.r.t. $\varepsilon$.

A classical article for the formal homogenization of the microscopic bidomain model - when the conductivities $\sigma_{\alpha}$ are strictly positive constants - is [43]. In [17], the homogenization of the microscopic bidomain equations (with constants conductivities) is presented using formal asymptotic analysis. It should also be noted that in $[6,50]$, the same type of results have be proven using the theory of $\Gamma$-convergence in some simplified situations. The approach presented below uses the 2-scale convergence method (see [3]) to extend the results obtained in $[6,50]$. As typical for this kind of homogenization problem, we adopt the following approach:

1. Nondimensionalization of the problem. The microscopic bidomain equations are scaled in space and time, and written unitless. Using the characteristic values of the physical parameters of our problem (cells size, conductivities, ionic current,...), a small parameter $\varepsilon$ is introduced in the equations and in the geometry.

2. Uniform estimate of solutions. Using energy estimates, norms of the solutions - as well as norms of the non-linear terms - are uniformly bounded with respect to the small parameter $\varepsilon$.

3. Two-scale convergence. The limit equations are deduced by application of the 2 -scale convergence theory. One of the main difficulties of this step is the convergence analysis of the non-linear terms. It relies on the one-sided Lipschitz assumption (Assumption 8).

Although the elimination of the electrostatic potentials $u_{i}$ and $u_{e}$ was useful to simplify the analysis of the microscopic bidomain equations, we must re-introduce these unknowns for the homogenization process. The reason is that - to the best of our knowledge - only local in space differential operators are adapted to the 2-scale homogenization process and the operator $\mathcal{A}$ does not enter into this category of operators.

\subsection{Nondimensionalization of the problem}

The nondimensionalization of the system is necessary in order to understand the relative amplitudes of the different terms. In the literature, few works in that direction have been 
carried out, although we can cite [54] for a nondimensionalization analysis of the ionic term $I_{i o n}$ and [43] for the analysis in the context of homogenization. We define $L_{0}$ as a characteristic length of the heart and $T_{0}$ as a characteristic time of a cardiac cycle. In the same spirit, we denote by $\Sigma_{0}$ a characteristic conductivity, $C_{0}$ a characteristic membrane capacitance, $V_{\max }$ and $V_{\min }$ characteristic upper and lower bounds for the transmembrane potential and $W_{0}$ a characteristic value of the gating variable. We set

$$
\left\{\begin{array}{l}
u_{e}(\vec{x}, t)=\left(V_{\max }-V_{\text {min }}\right) \tilde{u}_{e}\left(\frac{\vec{x}}{L_{0}}, \frac{t}{T_{0}}\right), \\
u_{i}(\vec{x}, t)=\left(V_{\max }-V_{\min }\right) \tilde{u}_{i}\left(\frac{\vec{x}}{L_{0}}, \frac{t}{T_{0}}\right)+V_{\text {min }} \\
w(\vec{x}, t)=W_{0} \tilde{w}\left(\frac{\vec{x}}{L_{0}}, \frac{t}{T_{0}}\right), \\
C_{m}=C_{0} \tilde{C}_{m} \\
\overrightarrow{\vec{\sigma}}_{\alpha}=\Sigma_{0} \tilde{\overrightarrow{\vec{\sigma}}}_{\alpha} .
\end{array}\right.
$$

We assume that the contribution of $I_{i o n}$ and $I_{a p p}$ are of the same order, namely, there exists a characteristic current amplitude $I_{0}$ such that

$$
\left\{\begin{array}{l}
I_{\text {ion }}\left(\vec{x}, u_{i}-u_{e}, w\right)=I_{0} \tilde{I}_{i o n}\left(\frac{\vec{x}}{L_{0}}, \tilde{u}_{i}-\tilde{u}_{e}, \tilde{w}\right), \\
I_{a p p}=I_{0} \tilde{I}_{a p p} .
\end{array}\right.
$$

In the same way, we assume that $g$ can be defined using a normalized function $\tilde{g}$ and a characteristic amplitude $G_{0}$ such that

$$
g\left(\vec{x}, u_{i}-u_{e}, w\right)=G_{0} \tilde{g}\left(\frac{\vec{x}}{L_{0}}, \tilde{u}_{i}-\tilde{u}_{e}, \tilde{w}\right) .
$$

All quantities denoted by a tilde are dimensionless quantities. We obtain from (11), the dimensionless system

$$
\left\{\begin{aligned}
\vec{\nabla}_{\vec{x}} \cdot\left(\tilde{\overrightarrow{\vec{\sigma}}}_{\alpha} \vec{\nabla}_{\vec{x}} \tilde{u}_{\alpha}\right) & =0 & \tilde{\Omega}_{\alpha}, \\
\tilde{\overrightarrow{\vec{\sigma}}}_{i} \vec{\nabla}_{\vec{x}} \tilde{u}_{i} \cdot \vec{n}_{i} & =\tilde{\overrightarrow{\vec{\sigma}}}_{e} \vec{\nabla}_{\vec{x}} \tilde{u}_{e} \cdot \vec{n}_{i} & \tilde{\Gamma}_{m}, \\
\tilde{\overrightarrow{\vec{\sigma}}}_{i} \vec{\nabla}_{\vec{x}} \tilde{u}_{i} \cdot \vec{n}_{i} & =-\frac{L_{0} I_{0}}{\Sigma_{0} U_{0}} \tilde{I}_{i o n}\left(\cdot, \tilde{u}_{i}-\tilde{u}_{e}, \tilde{w}\right)+\frac{L_{0} I_{0}}{\Sigma_{0} U_{0}} \tilde{I}_{a p p}-\frac{L_{0} C_{0}}{\Sigma_{0} T_{0}} \tilde{C}_{m} \partial_{t}\left(\tilde{u}_{i}-\tilde{u}_{e}\right) & \tilde{\Gamma}_{m}, \\
\partial_{t} \tilde{w} & =-\frac{T_{0} G_{0}}{W_{0}} \tilde{g}\left(\tilde{u}_{i}-\tilde{u}_{e}, \tilde{w}\right) & \tilde{\Gamma}_{m},
\end{aligned}\right.
$$

where $\tilde{\Omega}_{\alpha}$ and $\tilde{\Gamma}_{m}$ are rescaled by $L_{0}$ and where $U_{0}=V_{\max }-V_{\min }$. The same nondimensionalization process is used to define boundary conditions along $\partial \tilde{\Omega}$ using the boundary conditions given by Equation (3).

We now define $\varepsilon$ - the parameter which tends to zero in the homogenization process as the ratio between the maximal length of a cell (of the order $10^{-4} \mathrm{~m}$ ) and $L_{0}$ (equals to $\left.10^{-1} \mathrm{~m}\right)$. This implies that $\varepsilon$ is of the order of $10^{-3}$. The dimensionless quantity

$$
\frac{L_{0} C_{0}}{\Sigma_{0} T_{0}}
$$

- with $T_{0}$ of the order of $1 \mathrm{~s}, C_{0}$ of $10^{-2} \mathrm{~F} . \mathrm{m}^{-2}$ and $\Sigma_{0}$ of $1 \mathrm{~S} . \mathrm{m}^{-1}$ - is of the same order of $\varepsilon$ and can be set to $\varepsilon$ by a small modification of the reference quantities. The term $U_{0}$ is of order $10^{-1} \mathrm{~V}$ and the term $I_{0}$ of order $10^{-3} \mathrm{~A} . \mathrm{m}^{-2}$ (this is the typical order of magnitude of $\left.I_{a p p}\right)$, therefore we have

$$
\frac{L_{0} I_{0}}{\Sigma_{0} U_{0}}
$$


of the order of $\varepsilon$ and set it to $\varepsilon$. Finally, up to a small change in the definition of $g$, we assume that $T_{0} G_{0} / W_{0}$ is of order 1 . The fact that $\varepsilon$ is small means that the microscopic scale and the macroscopic scale are well separated. For the sake of clarity, we do not keep the tilde notation but we write explicitly the dependence in $\varepsilon$. To study the mathematical properties of this problem, we consider the family of problems parametrized by $\varepsilon>0$, and we will characterize the limit equation as $\varepsilon$ tends to zero.

We will use the results of the 2 -scale convergence, see [3]. This method has been used in many fields of science and engineering. The main assumption of the 2 -scale convergence to obtain a well defined limit problem, is that the domain - in which the equations are solved is periodic.

We denote by $Y$ the open reference domain that is used to define the idealized microstructure corresponding to the periodic arrangement of cardiac cells. This micro-structure is decomposed into two open connected subdomains: the intracellular part $Y_{i}$ and the extracellular part $Y_{e}$. We have

$$
Y_{i} \cap Y_{e}=\emptyset, \quad \bar{Y}=\bar{Y}_{i} \cup \bar{Y}_{e}
$$

The intra- and the extra-cellular domains are separated by $\Gamma_{Y}$. The global position vector is denoted by $\vec{x}$ and the local position vector by $\vec{y}$. We define the domain $\bar{\Omega}=\overline{\Omega_{i}^{\varepsilon}} \cup \overline{\Omega_{e}^{\varepsilon}}$ by $\varepsilon$-periodicity and we denote by $\Gamma_{m}^{\varepsilon}$ the boundary between the intra- and the extra-cellular domains $\Omega_{i}^{\varepsilon}$ and $\Omega_{e}^{\varepsilon}$. More precisely we assume that $\Omega_{i}^{\varepsilon}$ and $\Omega_{e}^{\varepsilon}$ are the union of entire cells and we have

$$
\Omega_{\alpha}^{\varepsilon}=\bigcup_{k}\left(\varepsilon Y_{\alpha}+\varepsilon \vec{w}_{k}\right) \text { and then } \Gamma_{m}^{\varepsilon}=\bigcup_{k}\left(\varepsilon \Gamma_{Y}+\varepsilon \vec{w}_{k}\right),
$$

where $\vec{w}_{k}$ is the vector corresponding to the translation between the considered cell and the reference cell. By definition, we have $\vec{w}_{0}=\overrightarrow{0}$. Note that by construction, from any macroscopic position vector $\vec{x}$, one can deduce a corresponding position $\vec{y}$ in the reference periodic cell by $\vec{y}=\vec{x} / \varepsilon$. We assume that the diffusion tensors depend on the two scales using $\varepsilon$-independent tensor fields

$$
\overrightarrow{\vec{\sigma}}_{\alpha}^{\varepsilon}(\vec{x})=\overrightarrow{\vec{\sigma}}_{\alpha}\left(\vec{x}, \frac{\vec{x}}{\varepsilon}\right) .
$$

The objective is to homogenize the following problem, i.e. study the convergence - when $\varepsilon$ tends to zero - of the solutions of the microscopic bidomain model,

$$
\left\{\begin{array}{rlrl}
\vec{\nabla}_{\vec{x}} \cdot\left(\overrightarrow{\vec{\sigma}}_{\alpha}^{\varepsilon} \vec{\nabla}_{\vec{x}} u_{\alpha}^{\varepsilon}\right) & =0 & \Omega_{\alpha}^{\varepsilon}, \\
\overrightarrow{\vec{\sigma}}_{i}^{\varepsilon} \vec{\nabla}_{\vec{x}} u_{i}^{\varepsilon} \cdot \vec{n}_{i} & =\overrightarrow{\vec{\sigma}}_{e}^{\varepsilon} \vec{\nabla}_{\vec{x}} u_{e}^{\varepsilon} \cdot \vec{n}_{i}, & \Gamma_{m}^{\varepsilon} \\
\overrightarrow{\vec{\sigma}}_{i}^{\varepsilon} \vec{\nabla}_{\vec{x}} u_{i}^{\varepsilon} \cdot \vec{n}_{i} & =-\varepsilon C_{m} \partial_{t}\left(u_{i}^{\varepsilon}-u_{e}^{\varepsilon}\right)-\varepsilon I_{i o n}\left(u_{i}^{\varepsilon}-u_{e}^{\varepsilon}, w^{\varepsilon}\right)+\varepsilon I_{a p p}^{\varepsilon} & \Gamma_{m}^{\varepsilon}, \\
\partial_{t} w^{\varepsilon} & =-g\left(u_{i}^{\varepsilon}-u_{e}^{\varepsilon}, w^{\varepsilon}\right), & & \Gamma_{m}^{\varepsilon} .
\end{array}\right.
$$

The boundary conditions along $\partial \Omega$ read

$$
\overrightarrow{\vec{\sigma}}_{i}^{\varepsilon} \vec{\nabla}_{\vec{x}} u_{\alpha}^{\varepsilon} \cdot \vec{n}_{\alpha}=0 \quad \partial \Omega \cap \partial \Omega_{\alpha}^{\varepsilon},
$$

and the initial conditions are

$$
u_{i}^{\varepsilon}(\cdot, 0)-u_{e}^{\varepsilon}(\cdot, 0)=V_{m}^{0, \varepsilon}, \quad w^{\varepsilon}(\cdot, 0)=w^{0, \varepsilon}, \quad \Gamma_{m}^{\varepsilon} .
$$

Finally, for the definition of a unique extracellular electric potential, we impose

$$
\int_{\Gamma_{m}^{\varepsilon}} u_{e}^{\varepsilon} d \gamma=0
$$




\subsection{Uniform estimate of the solutions}

The homogenization process - i.e. the analysis of the limit process when $\varepsilon$ tends to 0 requires norm estimates of the solution that are independent of the parameter $\varepsilon$. This is the objective of this subsection.

A variational equation for unknowns $u_{\alpha}^{\varepsilon}$ can be directly deduced from the partial differential equations (45) and (46). It reads, for almost all $t \in[0, T]$,

$$
\begin{aligned}
\left(\vec{\sigma}_{i}^{\varepsilon} \vec{\nabla}_{\vec{x}} u_{i}^{\varepsilon}, \vec{\nabla}_{\vec{x}} v_{i}^{\varepsilon}\right)_{\Omega_{i}^{\varepsilon}}+\left(\vec{\sigma}_{e}^{\varepsilon} \vec{\nabla}_{\vec{x}} u_{e}^{\varepsilon}, \vec{\nabla}_{\vec{x}} v_{e}^{\varepsilon}\right)_{\Omega_{e}^{\varepsilon}}+\varepsilon C_{m}\left\langle\frac{\partial\left(u_{i}^{\varepsilon}-u_{e}^{\varepsilon}\right)}{\partial t}, v_{i}^{\varepsilon}-v_{e}^{\varepsilon}\right\rangle_{\Gamma_{m}^{\varepsilon}} & \\
& +\varepsilon\left\langle I_{i o n}\left(u_{i}^{\varepsilon}-u_{e}^{\varepsilon}, w^{\varepsilon}\right), v_{i}^{\varepsilon}-v_{e}^{\varepsilon}\right\rangle_{\Gamma_{m}^{\varepsilon}}=\varepsilon\left(I_{a p p}^{\varepsilon}, v_{i}^{\varepsilon}-v_{e}^{\varepsilon}\right)_{\Gamma_{m}^{\varepsilon}},
\end{aligned}
$$

for all $\left(v_{i}^{\varepsilon}, v_{e}^{\varepsilon}\right) \in H^{1}\left(\Omega_{i}^{\varepsilon}\right) \times H^{1}\left(\Omega_{e}^{\varepsilon}\right)$. We can formally derive an energy estimate by assuming that $u_{\alpha}^{\varepsilon}$ is regular in time and by taking $v_{\alpha}^{\varepsilon}=u_{\alpha}^{\varepsilon}(\cdot, t)$ in (49). Since, by definition $V_{m}^{\varepsilon}=$ $u_{i}^{\varepsilon}-u_{e}^{\varepsilon}$ on $\Gamma_{m}^{\varepsilon}$, we obtain

$$
\begin{aligned}
\left\|\vec{\nabla}_{\vec{x}} u_{i}^{\varepsilon}\right\|_{L_{\sigma_{i}}^{2}}^{2}+\left\|\vec{\nabla}_{\vec{x}} u_{e}^{\varepsilon}\right\|_{L_{\sigma_{e}}^{2}}^{2}+\varepsilon \frac{C_{m}}{2} \frac{d}{d t} \| & V_{m}^{\varepsilon} \|_{L^{2}\left(\Gamma_{m}^{\varepsilon}\right)}^{2} \\
& +\varepsilon\left\langle I_{i o n}\left(V_{m}^{\varepsilon}, w^{\varepsilon}\right), V_{m}^{\varepsilon}\right\rangle_{\Gamma_{m}^{\varepsilon}}=\varepsilon\left(I_{a p p}^{\varepsilon}, V_{m}^{\varepsilon}\right)_{\Gamma_{m}^{\varepsilon}} .
\end{aligned}
$$

Before integrating (50) with respect to time, we multiply the equation by $e^{-\lambda t}$, where $\lambda$ is a positive constant which will be determined in what follows. The third term of (50) becomes

$$
\begin{aligned}
\varepsilon \frac{C_{m}}{2} \int_{0}^{t} e^{-\lambda s} \frac{d}{d t}\left\|V_{m}^{\varepsilon}\right\|_{L^{2}\left(\Gamma_{m}^{\varepsilon}\right)}^{2} \mathrm{~d} s=\varepsilon \frac{C_{m}}{2} e^{-\lambda t}\left\|V_{m}^{\varepsilon}(t)\right\|_{L^{2}\left(\Gamma_{m}^{\varepsilon}\right)}^{2}-\varepsilon \frac{C_{m}}{2}\left\|V_{m}^{\varepsilon}(0)\right\|_{L^{2}\left(\Gamma_{m}^{\varepsilon}\right)}^{2} \\
+\varepsilon \frac{\lambda C_{m}}{2} \int_{0}^{t} e^{-\lambda s}\left\|V_{m}^{\varepsilon}\right\|_{L^{2}\left(\Gamma_{m}^{\varepsilon}\right)}^{2} \mathrm{~d} s .
\end{aligned}
$$

Similarly, for all $\mu>0$, one can deduce that

$$
\begin{aligned}
\varepsilon \frac{\mu}{2} \int_{0}^{t} e^{-\lambda s} \frac{d}{d t}\left\|w^{\varepsilon}\right\|_{L^{2}\left(\Gamma_{m}^{\varepsilon}\right)}^{2} \mathrm{~d} s= & \varepsilon \frac{\mu}{2} e^{-\lambda t}\left\|w^{\varepsilon}(t)\right\|_{L^{2}\left(\Gamma_{m}^{\varepsilon}\right)}^{2}-\varepsilon \frac{\mu}{2}\left\|w^{0, \varepsilon}\right\|_{L^{2}\left(\Gamma_{m}^{\varepsilon}\right)}^{2} \\
& +\varepsilon \frac{\lambda \mu}{2} \int_{0}^{t} e^{-\lambda s}\left\|w^{\varepsilon}\right\|_{L^{2}\left(\Gamma_{m}^{\varepsilon}\right)}^{2} \mathrm{~d} s \\
& =-\varepsilon \mu \int_{0}^{t} e^{-\lambda s}\left(g\left(V_{m}^{\varepsilon}, w^{\varepsilon}\right), w^{\varepsilon}\right)_{\Gamma_{m}^{\varepsilon}} d s .
\end{aligned}
$$

Note that in the previous equation, we have introduced the scalar $\mu$ in order to use Assumption 6 . Then using the two previous equations as well as (50), we obtain

$$
\begin{aligned}
& \mathcal{E}_{\lambda, \mu}\left(u_{i}^{\varepsilon}, u_{e}^{\varepsilon}, w^{\varepsilon}, t\right)-\mathcal{E}_{\lambda, \mu}\left(u_{i}^{\varepsilon}, u_{e}^{\varepsilon}, w^{\varepsilon}, 0\right) \\
&+\varepsilon \int_{0}^{t} e^{-\lambda s}\left[\left\langle I_{i o n}\left(V_{m}^{\varepsilon}, w^{\varepsilon}\right), V_{m}^{\varepsilon}\right\rangle_{\Gamma_{m}^{\varepsilon}}+\frac{\lambda C_{m}}{2}\left\|V_{m}^{\varepsilon}\right\|_{L^{2}\left(\Gamma_{m}^{\varepsilon}\right)}^{2}\right] \mathrm{d} s \\
&+\varepsilon \int_{0}^{t} e^{-\lambda s}\left[\mu\left(g\left(V_{m}^{\varepsilon}, w^{\varepsilon}\right), w^{\varepsilon}\right)_{\Gamma_{m}^{\varepsilon}}+\frac{\lambda \mu}{2}\left\|w^{\varepsilon}\right\|_{L^{2}\left(\Gamma_{m}^{\varepsilon}\right)}^{2}\right] \mathrm{d} s=\varepsilon \int_{0}^{t} e^{-\lambda s}\left(I_{a p p}^{\varepsilon}, V_{m}^{\varepsilon}\right)_{\Gamma_{m}^{\varepsilon}} \mathrm{d} s,
\end{aligned}
$$

where the term $\mathcal{E}_{\lambda, \mu}$ is the energy associated to the system and is defined by

$$
\begin{aligned}
\mathcal{E}_{\lambda, \mu}\left(u_{i}^{\varepsilon}, u_{e}^{\varepsilon}, w^{\varepsilon}, t\right)=\varepsilon \frac{C_{m}}{2} e^{-\lambda t}\left\|V_{m}^{\varepsilon}(t)\right\|_{L^{2}\left(\Gamma_{m}^{\varepsilon}\right)}^{2}+\varepsilon \frac{\mu}{2} e^{-\lambda t}\left\|w^{\varepsilon}(t)\right\|_{L^{2}\left(\Gamma_{m}^{\varepsilon}\right)}^{2} \\
+\int_{0}^{t} e^{-\lambda s}\left\|\vec{\nabla}_{\vec{x}} u_{i}^{\varepsilon}\right\|_{L_{\sigma_{i}}^{2}}^{2} \mathrm{~d} s+\int_{0}^{t} e^{-\lambda s}\left\|\vec{\nabla}_{\vec{x}} u_{e}^{\varepsilon}\right\|_{L_{\sigma_{e}}^{2}}^{2} \mathrm{~d} s .
\end{aligned}
$$


To shorten the presentation, we omit the reference to the physical quantities in the definition of the energy, i.e. in what follows, we introduce the notation

$$
\mathcal{E}_{\lambda, \mu}^{\varepsilon}(t)=\mathcal{E}_{\lambda, \mu}\left(u_{i}^{\varepsilon}, u_{e}^{\varepsilon}, w^{\varepsilon}, t\right) .
$$

For $\mu>0$ given by Assumption 6, we assume that $\lambda$ is sufficiently large, more precisely it should satisfy

$$
\min \left(\frac{\lambda C_{m}}{2}, \frac{\lambda \mu}{2}\right) \geq C_{I}
$$

where $C_{I}$ is the positive scalar appearing in (30) and is independent of $\varepsilon$. Using Assumption 6 and (51). We then obtain the first energy estimate

$$
\mathcal{E}_{\lambda, \mu}^{\varepsilon}(t) \leq \mathcal{E}_{\lambda, \mu}^{\varepsilon}(0)+\varepsilon \int_{0}^{t} e^{-\lambda s}\left(\left(I_{a p p}^{\varepsilon}, V_{m}^{\varepsilon}\right)_{\Gamma_{m}^{\varepsilon}}+C_{I}\left|\Gamma_{m}^{\varepsilon}\right|\right) \mathrm{d} s .
$$

Relation (51) is the energy relation that can be proven rigorously using a regularization process. Such derivations are done in the proof of Theorem 1 (see Remark 7 in Appendix 5). We also refer to the macroscopic bidomain model analysis of [8] for some related considerations. We are now in the position to state the first proposition of this subsection.

Proposition 5. There exist positive scalars $\mu, \lambda_{0}$ and $C$ independent of $\varepsilon$ such that, for all $\lambda \geq \lambda_{0}$ and for all $t \in[0, T]$, the solutions given by Theorem 1 satisfy

$$
\mathcal{E}_{\lambda, \mu}^{\varepsilon}(t) \leq \mathcal{E}_{\lambda, \mu}^{\varepsilon}(0)+C\left(1+\varepsilon^{1 / 2} \int_{0}^{t} e^{-\frac{\lambda}{2} s}\left\|I_{a p p}^{\varepsilon}\right\|_{L^{2}\left(\Gamma_{m}^{\varepsilon}\right)} d s\right)
$$

Proof. By noting that the last term of (54) can be estimated as follows

$$
\varepsilon \int_{0}^{t} e^{-\lambda s}\left(I_{a p p}^{\varepsilon}, V_{m}^{\varepsilon}\right)_{\Gamma_{m}^{\varepsilon}} \mathrm{d} s \leq\left(\frac{2 \varepsilon}{C_{m}}\right)^{1 / 2} \int_{0}^{t} e^{-\frac{\lambda}{2} s}\left\|I_{a p p}^{\varepsilon}\right\|_{L^{2}\left(\Gamma_{m}^{\varepsilon}\right)}\left[\mathcal{E}_{\lambda, \mu}^{\varepsilon}(s)\right]^{1 / 2} \mathrm{~d} s
$$

and that $\varepsilon\left|\Gamma_{m}^{\varepsilon}\right|$ is bounded uniformly w.r.t. $\varepsilon$, we can conclude using Gronwall's inequality (see [21], Theorem 5).

To obtain uniform estimates, we need the following assumption.

Assumption 11. Uniform estimates of the data. We assume that there exists a scalar $C>0$ independent of $\varepsilon$ such that

$$
\varepsilon^{1 / 2} \int_{0}^{T}\left\|I_{a p p}^{\varepsilon}\right\|_{L^{2}\left(\Gamma_{m}^{\varepsilon}\right)} d t \leq C \quad \text { and } \quad \varepsilon\left\|V_{m}^{0, \varepsilon}\right\|_{L^{2}\left(\Gamma_{m}^{\varepsilon}\right)}^{2}+\varepsilon\left\|w^{0, \varepsilon}\right\|_{L^{2}\left(\Gamma_{m}^{\varepsilon}\right)}^{2} \leq C .
$$

Now we introduce the following proposition which is the main result of this section.

Proposition 6. If Assumption 11 holds, there exists a positive scalar $C$ independent of $\varepsilon$, such that solutions of the bidomain equations - given by Theorem 1 - satisfy

$$
\int_{0}^{T}\left\|u_{i}^{\varepsilon}\right\|_{H^{1}\left(\Omega_{i}^{\varepsilon}\right)}^{2} d t+\int_{0}^{T}\left\|u_{e}^{\varepsilon}\right\|_{H^{1}\left(\Omega_{e}^{\varepsilon}\right)}^{2} d t \leq C
$$

and

$$
\varepsilon \int_{0}^{T} \int_{\Gamma_{m}^{\varepsilon}}\left|I_{i o n}\left(V_{m}^{\varepsilon}, w^{\varepsilon}\right)\right|^{4 / 3} d \gamma d t+\varepsilon \int_{0}^{T} \int_{\Gamma_{m}^{\varepsilon}}\left|g\left(V_{m}^{\varepsilon}, w^{\varepsilon}\right)\right|^{2} d \gamma d t \leq C
$$

In order to simplify the proof of Proposition 6, we will introduce one preliminary corollary and two preliminary lemmas. Our starting point is Proposition 5 together with Assumption 11 that provides uniform bounds on the data. As a direct consequence of this assumption and using the equivalence between $\|\cdot\|_{L^{2}}$ and $\|\cdot\|_{L_{\sigma_{\alpha}}^{2}}$, we have the following corollary. 
Corollary 3. If Assumption 11 holds, there exists a positive scalar $C$ independent of $\varepsilon$, such that solutions of the bidomain equations - given by Theorem 1 - satisfy

$$
\int_{0}^{T}\left\|\vec{\nabla}_{\vec{x}} u_{i}^{\varepsilon}\right\|_{L^{2}\left(\Omega_{i}^{\varepsilon}\right)}^{2} d t+\int_{0}^{T}\left\|\vec{\nabla}_{\vec{x}} u_{e}^{\varepsilon}\right\|_{L^{2}\left(\Omega_{e}^{\varepsilon}\right)}^{2} d t \leq C
$$

and for all $t \in[0, T]$,

$$
\varepsilon\left\|V_{m}^{\varepsilon}(t)\right\|_{L^{2}\left(\Gamma_{m}^{\varepsilon}\right)}^{2}+\varepsilon\left\|w^{\varepsilon}(t)\right\|_{L^{2}\left(\Gamma_{m}^{\varepsilon}\right)}^{2} \leq C .
$$

Corollary 3 is still not sufficient for our purpose since we need an estimation of the intraand extra-cellular potentials in the $L^{2}$-norm in $\Omega_{i}^{\varepsilon}$ and $\Omega_{e}^{\varepsilon}$ respectively. To do so, we need a Poincaré-Wirtinger inequality and a trace inequality that should take into account the geometry dependence in $\varepsilon$. Such inequalities are given in [7], Corollary B.1 and Lemma C.1. They are given in dimension 2 but they can be extended to the 3 -dimensional setting. With our notations, these inequalities are given in the following lemma.

Lemma 3. There exists a constant $C$ independent of $\varepsilon$ such that for all $v_{e}^{\varepsilon} \in H^{1}\left(\Omega_{e}^{\varepsilon}\right)$, we have

$$
\left\|v_{e}^{\varepsilon}-\frac{1}{\left|\Omega_{e}^{\varepsilon}\right|} \int_{\Omega_{e}^{\varepsilon}} v_{e}^{\varepsilon} d \vec{x}\right\|_{L^{2}\left(\Omega_{e}^{\varepsilon}\right)} \leq C\left\|\vec{\nabla}_{\vec{x}} v_{e}^{\varepsilon}\right\|_{L^{2}\left(\Omega_{e}^{\varepsilon}\right)}
$$

and

$$
\left\|v_{e}^{\varepsilon}\right\|_{L^{2}\left(\Gamma_{m}^{\varepsilon}\right)}^{2} \leq C \varepsilon^{-1}\left\|v_{e}^{\varepsilon}\right\|_{L^{2}\left(\Omega_{e}^{\varepsilon}\right)}^{2}+C \varepsilon\left\|\vec{\nabla}_{\vec{x}} v_{e}^{\varepsilon}\right\|_{L^{2}\left(\Omega_{e}^{\varepsilon}\right)}^{2}
$$

Note that inequality (58) is no longer true if the domain $\Omega_{e}^{\varepsilon}$ does not satisfy (44), i.e. if $\Omega$ is not the union of entire cells for all $\varepsilon$ (which allows non-connected extra-cellular subdomains to appear at the boundary of the domain for some sequences of $\varepsilon$ ). Moreover, we also need a Poincaré-like inequality to bound the $L^{2}$-norm of the solution inside the intraand the extra-cellular domains. Such an inequality can be found in [7] Lemma C.2, and in our context, it is given in the following lemma.

Lemma 4. There exists a constant $C$ independent of $\varepsilon$ such that for all $v_{\alpha}^{\varepsilon} \in H^{1}\left(\Omega_{\alpha}^{\varepsilon}\right)$,

$$
\left\|v_{\alpha}^{\varepsilon}\right\|_{L^{2}\left(\Omega_{\alpha}^{\varepsilon}\right)}^{2} \leq C \varepsilon\left\|v_{\alpha}^{\varepsilon}\right\|_{L^{2}\left(\Gamma_{m}^{\varepsilon}\right)}^{2}+C \varepsilon^{2}\left\|\vec{\nabla}_{\vec{x}} v_{\alpha}^{\varepsilon}\right\|_{L^{2}\left(\Omega_{\alpha}^{\varepsilon}\right)}^{2} .
$$

Finally collecting the results of Corollary 3 and Lemmas 3 and 4, we can prove Proposition 6.

\section{Proof. (Proof of Proposition 6)}

Step 1: A preliminary inequality. To simplify the following computations, we introduce the linear forms $m_{\Omega}$ and $m_{\Gamma}$ defined for all $v_{e}^{\varepsilon} \in H^{1}\left(\Omega_{e}^{\varepsilon}\right)$ by

$$
m_{e}\left(v_{e}^{\varepsilon}\right)=\frac{1}{\left|\Omega_{e}^{\varepsilon}\right|} \int_{\Omega_{e}^{\varepsilon}} v_{e}^{\varepsilon} d \vec{x} \quad \text { and } \quad m_{\Gamma}\left(v_{e}^{\varepsilon}\right)=\frac{1}{\left|\Gamma_{m}^{\varepsilon}\right|} \int_{\Gamma_{m}^{\varepsilon}} v_{e}^{\varepsilon} d \gamma
$$

For all $v_{e}^{\varepsilon} \in H^{1}\left(\Omega_{e}^{\varepsilon}\right)$, we have

$$
\begin{aligned}
\left\|v_{e}^{\varepsilon}-m_{e}\left(v_{e}^{\varepsilon}\right)\right\|_{L^{2}\left(\Gamma_{m}^{\varepsilon}\right)}^{2} & =\left\|v_{e}^{\varepsilon}-m_{\Gamma}\left(v_{e}^{\varepsilon}\right)-m_{e}\left(v_{e}^{\varepsilon}-m_{\Gamma}\left(v_{e}^{\varepsilon}\right)\right)\right\|_{L^{2}\left(\Gamma_{m}^{\varepsilon}\right)}^{2} \\
& =\left\|v_{e}^{\varepsilon}-m_{\Gamma}\left(v_{e}^{\varepsilon}\right)\right\|_{L^{2}\left(\Gamma_{m}^{\varepsilon}\right)}^{2}+\left\|m_{e}\left(v_{e}^{\varepsilon}-m_{\Gamma}\left(v_{e}^{\varepsilon}\right)\right)\right\|_{L^{2}\left(\Gamma_{m}^{\varepsilon}\right)}^{2} \\
& -2 \int_{\Gamma_{m}^{\varepsilon}}\left(v_{e}^{\varepsilon}-m_{\Gamma}\left(v_{e}^{\varepsilon}\right)\right) m_{e}\left(v_{e}^{\varepsilon}-m_{\Gamma}\left(v_{e}^{\varepsilon}\right)\right) d \gamma
\end{aligned}
$$

Now observing that the last term vanishes hence, for all $v_{e}^{\varepsilon} \in H^{1}\left(\Omega_{e}^{\varepsilon}\right)$, we have

$$
\left\|v_{e}^{\varepsilon}-m_{\Gamma}\left(v_{e}^{\varepsilon}\right)\right\|_{L^{2}\left(\Gamma_{m}^{\varepsilon}\right)}^{2} \leq\left\|v_{e}^{\varepsilon}-m_{e}\left(v_{e}^{\varepsilon}\right)\right\|_{L^{2}\left(\Gamma_{m}^{\varepsilon}\right)}^{2} .
$$


Step 2: Uniform estimates of the potentials. Using the trace inequality (59) (applied to $\left.v_{e}^{\varepsilon}-m_{e}\left(v_{e}^{\varepsilon}\right)\right)$ and inequality (61) we find

$$
\varepsilon\left\|v_{e}^{\varepsilon}-m_{\Gamma}\left(v_{e}^{\varepsilon}\right)\right\|_{L^{2}\left(\Gamma_{m}^{\varepsilon}\right)}^{2} \leq C\left\|v_{e}^{\varepsilon}-m_{e}\left(v_{e}^{\varepsilon}\right)\right\|_{L^{2}\left(\Omega_{e}^{\varepsilon}\right)}^{2}+\varepsilon^{2}\left\|\vec{\nabla}_{\vec{x}} v_{e}^{\varepsilon}\right\|_{L^{2}\left(\Omega_{e}^{\varepsilon}\right)}^{2} .
$$

Thanks to the Poincaré-Wirtinger inequality (58), we conclude that there exists $C$ independent of $\varepsilon$ such that

$$
\varepsilon\left\|v_{e}^{\varepsilon}-m_{\Gamma}\left(v_{e}^{\varepsilon}\right)\right\|_{L^{2}\left(\Gamma_{m}^{\varepsilon}\right)}^{2} \leq C\left\|\vec{\nabla}_{\vec{x}} v_{e}^{\varepsilon}\right\|_{L^{2}\left(\Omega_{e}^{\varepsilon}\right)}^{2} .
$$

Now setting $v_{e}^{\varepsilon}=u_{e}^{\varepsilon}$ in the previous equation (remind that $m_{\Gamma}\left(u_{e}^{\varepsilon}\right)=0$ ), integrating with respect to time and using the estimation of Corollary 3 , we find

$$
\varepsilon \int_{0}^{T}\left\|u_{e}^{\varepsilon}\right\|_{L^{2}\left(\Gamma_{m}^{\varepsilon}\right)}^{2} d s \leq C
$$

where $C$ is another constant independent of $\varepsilon$. With the estimate (57) of Corollary 3, we also have

$$
\varepsilon \int_{0}^{T}\left\|u_{i}^{\varepsilon}\right\|_{L^{2}\left(\Gamma_{m}^{\varepsilon}\right)}^{2} d s \leq C
$$

where $C$ is another constant independent of $\varepsilon$. Finally, we can use Lemma 4 to obtain the estimate (55).

Step 3: Uniform estimates of the non-linear terms. It is also important to obtain a uniform estimate on the term $I_{i o n}\left(u_{i}^{\varepsilon}-u_{i}^{\varepsilon}, w^{\varepsilon}\right)$ and on the term $g\left(u_{i}^{\varepsilon}-u_{i}^{\varepsilon}, w^{\varepsilon}\right)$ in the appropriate norms. From (51), we have

$$
\begin{aligned}
\varepsilon \int_{0}^{T} e^{-\lambda t}\left[\lambda\left|\Gamma_{m}^{\varepsilon}\right|+\right. & \left.\left\langle I_{i o n}\left(V_{m}^{\varepsilon}, w^{\varepsilon}\right), V_{m}^{\varepsilon}\right\rangle_{\Gamma_{m}^{\varepsilon}}+\frac{\lambda C_{m}}{2}\left\|V_{m}^{\varepsilon}\right\|_{L^{2}\left(\Gamma_{m}^{\varepsilon}\right)}^{2}\right] \mathrm{d} t \\
+ & \varepsilon \int_{0}^{T} e^{-\lambda t}\left[\mu\left(g\left(V_{m}^{\varepsilon}, w^{\varepsilon}\right), w^{\varepsilon}\right)_{\Gamma_{m}^{\varepsilon}}+\frac{\lambda \mu}{2}\left\|w^{\varepsilon}\right\|_{L^{2}\left(\Gamma_{m}^{\varepsilon}\right)}^{2}\right] \mathrm{d} t \\
& \leq \mathcal{E}_{\lambda, \mu}^{\varepsilon}(0)+\varepsilon \int_{0}^{T} e^{-\lambda t}\left(\left(I_{a p p}^{\varepsilon}, V_{m}^{\varepsilon}\right)_{\Gamma_{m}^{\varepsilon}}+C_{I}\left|\Gamma_{m}^{\varepsilon}\right|\right) \mathrm{d} t \equiv R_{\varepsilon}(T),
\end{aligned}
$$

then the right hand side of the equation above (denoted $R_{\varepsilon}(T)$ ) can be estimated as follows

$$
R_{\varepsilon}(T) \leq C\left(1+\varepsilon\left\|V_{m}^{0, \varepsilon}\right\|_{L^{2}\left(\Gamma_{m}^{\varepsilon}\right)}^{2}+\varepsilon\left\|w^{0, \varepsilon}\right\|_{L^{2}\left(\Gamma_{m}^{\varepsilon}\right)}^{2}\right)+\varepsilon C \sup _{t \in[0, T]}\left\|V_{m}^{\varepsilon}(t)\right\|_{L^{2}\left(\Gamma_{m}^{\varepsilon}\right)} \int_{0}^{T}\left\|I_{a p p}^{\varepsilon}\right\|_{L^{2}\left(\Gamma_{m}^{\varepsilon}\right)}
$$

where we have used the property that $\varepsilon\left|\Gamma_{m}^{\varepsilon}\right|$ is bounded uniformly with respect to $\varepsilon$ and $C$ is a constant independent of $\varepsilon$. As a consequence of Assumption 11 and Corollary 3, we have that $R_{\varepsilon}(T)$ is uniformly bounded w.r.t. $\varepsilon$. Using Assumption 6, we know that there exists $\mu>0$ such that for $\lambda$ satisfying (53), the integrand of the left hand side of (62) is positive almost everywhere on $\Gamma_{m}^{\varepsilon}$. Therefore, bounding $e^{-\lambda t}$ by below, we deduce that

$$
\varepsilon \int_{0}^{T} \int_{\Gamma_{m}^{\varepsilon}}\left|\lambda+I_{i o n}\left(V_{m}^{\varepsilon}, w^{\varepsilon}\right) V_{m}^{\varepsilon}+\frac{\lambda C_{m}}{2}\left(V_{m}^{\varepsilon}\right)^{2}+\mu g\left(V_{m}^{\varepsilon}, w^{\varepsilon}\right) w^{\varepsilon}+\frac{\lambda \mu}{2}\left(w^{\varepsilon}\right)^{2}\right| d \gamma \mathrm{d} t \leq C
$$

where $C$ is another constant that depends on $e^{\lambda T}$ but is independent of $\varepsilon$. We must now study two distinct cases.

Step 3a: Assumption 7a holds. Since $g$ is Lipschitz we can show, with the estimate (57) that

$$
\varepsilon \int_{0}^{T} \int_{\Gamma_{m}^{\varepsilon}}\left|g\left(V_{m}^{\varepsilon}, w^{\varepsilon}\right)\right|^{2} d \gamma \mathrm{d} t \leq C
$$


where $C$ independent of $\varepsilon$. Therefore, we deduce from (63) that

$$
\varepsilon \int_{0}^{T} \int_{\Gamma_{m}^{\varepsilon}}\left|I_{i o n}\left(V_{m}^{\varepsilon}, w^{\varepsilon}\right) V_{m}^{\varepsilon}\right| d \gamma \mathrm{d} t \leq C,
$$

where $C$ is another scalar independent of $\varepsilon$. Therefore, using the growth condition (28) and Young's inequalities, we get

$$
\begin{aligned}
& \varepsilon \int_{0}^{T} \int_{\Gamma_{m}^{\varepsilon}}\left|I_{i o n}\left(V_{m}^{\varepsilon}, w^{\varepsilon}\right)\right|^{4 / 3} d \gamma \mathrm{d} t \\
& \leq \varepsilon C C_{\infty}^{1 / 3} \int_{0}^{T} \int_{\Gamma_{m}^{\varepsilon}}\left|I_{i o n}\left(V_{m}^{\varepsilon}, w^{\varepsilon}\right)\right|\left(\left|V_{m}^{\varepsilon}\right|+\left|w^{\varepsilon}\right|^{1 / 3}+1\right) d \gamma \mathrm{d} t \\
& \leq \varepsilon C C_{\infty}^{1 / 3} \int_{0}^{T} \int_{\Gamma_{m}^{\varepsilon}}\left|I_{i o n}\left(V_{m}^{\varepsilon}, w^{\varepsilon}\right) V_{m}^{\varepsilon}\right|+\frac{3}{2 \eta^{4 / 3}}\left|I_{i o n}\left(V_{m}^{\varepsilon}, w^{\varepsilon}\right)\right|^{4 / 3}+\frac{\eta^{4}}{4}\left|w^{\varepsilon}\right|^{4 / 3}+\frac{\eta^{4}}{4} d \gamma \mathrm{d} t
\end{aligned}
$$

where $\eta>0$ can be chosen arbitrarily and $C$ is another scalar independent of $\varepsilon$. Finally, since $\left|w^{\varepsilon}\right|^{4 / 3} \leq\left|w^{\varepsilon}\right|^{2}+4 / 27$ almost everywhere on $\Gamma_{m}^{\varepsilon}$, we can use estimates (64)-(57) and choose $\eta$ sufficiently large (but independent of $\varepsilon$ ) in order to obtain (56).

Step 3b: Assumption $7 b$ holds. Starting from (62) and using the first inequality in (32) we have

$$
\begin{aligned}
\varepsilon \int_{0}^{T} \int_{\Gamma_{m}^{\varepsilon}} \lambda+C_{1}\left|V_{m}^{\varepsilon}\right|^{4}-c_{1}\left(\left|V_{m}^{\varepsilon}\right|^{2}+1\right)+f_{2}\left(V_{m}^{\varepsilon}\right) V_{m}^{\varepsilon} w^{\varepsilon}+\frac{\lambda C_{m}}{2}\left(V_{m}^{\varepsilon}\right)^{2} & \\
& +\mu g\left(V_{m}^{\varepsilon}, w^{\varepsilon}\right) w^{\varepsilon}+\frac{\lambda \mu}{2}\left(w^{\varepsilon}\right)^{2} d \gamma \mathrm{d} t \leq C .
\end{aligned}
$$

Note that for $\lambda$ sufficiently large (but independent of $\varepsilon$ ), the integrand above is positive. Moreover, from Assumption $7 \mathrm{~b}$, we have, for all $\vec{x} \in \Omega$ and $(v, w) \in \mathbb{R}^{2}$,

$$
\left|f_{2}(\vec{x}, v, w) v w\right| \leq C\left(\frac{(|v|+1)^{2}|v|^{2}}{\eta}+\eta|w|^{2}\right),
$$

and

$$
|g(\vec{x}, v, w) w| \leq C\left(\frac{|v|^{4}}{\eta^{4 / 3}}+\left(1+\eta^{4}\right)|w|^{2}+1\right),
$$

for some $\eta>0$ and where $C$ is a positive constant independent of $\varepsilon$ and $\eta$. Therefore, by choosing $\eta$ large enough ( $\eta$ is independent of $\varepsilon$ ), one can show that there exists another positive constant $C$ independent of $\varepsilon$ such that

$$
\varepsilon \int_{0}^{T} \int_{\Gamma_{m}^{\varepsilon}}\left|V_{m}^{\varepsilon}\right|^{4} d \gamma \mathrm{d} t \leq C .
$$

The results of Proposition 6 are then a direct consequence of Assumption 5.

\subsection{Homogenization of the bidomain equations by 2-scale conver- gence}

The 2-scale convergence theory has been developed in the reference articles [3] and [45]. This mathematical tool justifies and deduces the homogenized problem in a single process. It is also well adapted to treat the case of perforated domains (in our case $\Omega_{i}$ and $\Omega_{e}$ can both be seen as perforated domains). The analysis of homogenization in a perforated domain presents some additional difficulties since the solutions are defined in domains whose geometry is not fixed. This issue is not new (see [14]) and it is addressed in [5] - using compactness results for sequence of functions defined in a family of perforated domains - or in [1], [15] 
and [13]. In the last two mentioned references, the periodic unfolding method is used. Such a method can be related to 2-scale convergence, as in [37] in which the homogenization of a reaction-diffusion problem is done using both techniques: the 2 -scale convergence gives the preliminary convergence results and the periodic unfolding method is used to deal with the reaction term. The treatment of the reaction terms - i.e. the non linear terms or the ionic terms in our context - is one of the main difficulty. To tackle this problem, we present an approach based upon the general ideas of the original article [3]. Finally, the last difficulty which is typical to biological tissue modeling, is that the non-linear terms of the equations - that correspond to exchange of ionic quantities at the membrane of a cell - lie on the boundary of the domain. Therefore the 2-scale convergence theory must be adapted and to do so, we use the results presented in [4] (see also [44] for related results).

We define $\Omega_{T}:=\Omega \times(0, T)$, and we introduce the space $C_{\sharp}^{0}(\bar{Y})$ of continuous periodic functions on the periodic cell $Y$ (up to the boundary) and $L_{\sharp}^{\infty}(Y)$ the space of essentially bounded periodic functions on $Y$.

Proposition 7. Let $\left\{u^{\varepsilon}\right\}$ be a sequence of functions in $L^{2}\left(\Omega_{T}\right)$ such that

$$
\int_{\Omega_{T}}\left|u^{\varepsilon}(\vec{x}, t)\right|^{2} d \vec{x} d t \leq C
$$

where $C$ does not depend on $\varepsilon$, then the sequence 2-scale converges to a limit $u_{0} \in L^{2}\left(\Omega_{T} \times Y\right)$, i.e. for any $\varphi \in C^{0}\left(\overline{\Omega_{T}} ; L_{\sharp}^{\infty}(Y)\right)$ we have, up to a subsequence,

$$
\lim _{\varepsilon \rightarrow 0} \int_{\Omega_{T}} u^{\varepsilon}(\vec{x}, t) \varphi(\vec{x}, t, \vec{x} / \varepsilon) d \vec{x} d t=\frac{1}{|Y|} \int_{\Omega_{T} \times Y} u_{0}(\vec{x}, t, \vec{y}) \varphi(\vec{x}, t, \vec{y}) d \vec{x} d t d \vec{y} .
$$

The same notion of weak convergence exists for a function defined on $\Gamma_{m}^{\varepsilon}$, and straightforward generalizations of the results in [4] lead to the following proposition.

Proposition 8. Let $\left\{u^{\varepsilon}\right\}$ be a sequence of functions in $L^{p}\left(\Gamma_{m}^{\varepsilon} \times(0, T)\right)$ with $p \in(1,+\infty)$ such that

$$
\varepsilon \int_{\Gamma_{m}^{\varepsilon} \times(0, T)}\left|u^{\varepsilon}(\vec{x}, t)\right|^{p} d \gamma d t \leq C
$$

where $C$ does not depend on $\varepsilon$, then the sequence 2-scale converges to a limit $u_{0} \in L^{p}\left(\Omega_{T} \times\right.$ $\left.\Gamma_{Y}\right)$, i.e. for any $\varphi \in C^{0}\left(\overline{\Omega_{T}} ; C_{\sharp}^{0}(\bar{Y})\right)$ we have, up to a subsequence,

$$
\lim _{\varepsilon \rightarrow 0} \int_{\Gamma_{m}^{\varepsilon} \times(0, T)} u^{\varepsilon}(\vec{x}, t) \varphi(\vec{x}, t, \vec{x} / \varepsilon) d \gamma d t=\frac{1}{|Y|} \int_{\Omega_{T} \times \Gamma_{Y}} u_{0}(\vec{x}, t, \vec{y}) \varphi(\vec{x}, t, \vec{y}) d \vec{x} d t d \gamma
$$

As previously mentioned, one of the main advantages of 2-scale convergence is the ability to analyze partial differential equations in a perforated domain by introducing simple extension operators. In our context $\Omega_{i}^{\varepsilon}$ and $\Omega_{e}^{\varepsilon}$ can be seen as perforated domains, therefore, following [3], we denote by $\sim$ the extension by zero in $\Omega$. More precisely, we define

$$
\widetilde{\vec{\nabla}_{\vec{x}} u_{i}^{\varepsilon}}=\left\{\begin{array}{cc}
\vec{\nabla}_{\vec{x}} u_{i}^{\varepsilon} & \Omega_{i}^{\varepsilon}, \\
0 & \Omega_{e}^{\varepsilon},
\end{array} \quad \widetilde{\vec{\nabla}_{\vec{x}} u_{e}^{\varepsilon}}=\left\{\begin{array}{cc}
0 & \Omega_{i}^{\varepsilon}, \\
\vec{\nabla}_{\vec{x}} u_{e}^{\varepsilon} & \Omega_{e}^{\varepsilon},
\end{array}\right.\right.
$$

and we define $\overrightarrow{\vec{\sigma}} \varepsilon$ by periodicity as follows

$$
\overrightarrow{\vec{\sigma}}(\vec{x}, \vec{y})=\left\{\begin{array}{ll}
\overrightarrow{\vec{\sigma}}_{i}(\vec{x}, \vec{y}) & \vec{y} \in Y_{i}, \\
\overrightarrow{\vec{\sigma}}_{e}(\vec{x}, \vec{y}) & \vec{y} \in Y_{e},
\end{array} \quad \text { and } \quad \overrightarrow{\vec{\sigma}}^{\varepsilon}(\vec{x})=\overrightarrow{\vec{\sigma}}\left(\vec{x}, \frac{\vec{x}}{\varepsilon}\right)\right.
$$

Using (49), one can see that the functions $\left(u_{i}^{\varepsilon}, u_{e}^{\varepsilon}\right)$ and $\left(\widetilde{\vec{\nabla}_{\vec{x}} u_{i}^{\varepsilon}}, \widetilde{\vec{\nabla}_{\vec{x}} u_{e}^{\varepsilon}}\right)$ satisfy, for all $v_{i}^{\varepsilon}, v_{e}^{\varepsilon} \in$ $H^{1}(\Omega)$ and for almost all $t \in(0, T)$,

$$
\begin{aligned}
& \left(\overrightarrow{\vec{\sigma}} \varepsilon \widetilde{\vec{\nabla}_{\vec{x}} u_{i}^{\varepsilon}}, \vec{\nabla}_{\vec{x}} v_{i}^{\varepsilon}\right)_{\Omega}+\left(\overrightarrow{\vec{\sigma}} \varepsilon \widetilde{\vec{\nabla}}_{\vec{x}} u_{e}^{\varepsilon}, \vec{\nabla}_{\vec{x}} v_{e}^{\varepsilon}\right)_{\Omega}+\varepsilon C_{m}\left\langle\frac{\partial\left(u_{i}^{\varepsilon}-u_{e}^{\varepsilon}\right)}{\partial t}, v_{i}^{\varepsilon}-v_{e}^{\varepsilon}\right\rangle_{\Gamma_{m}^{\varepsilon}} \\
& +\varepsilon\left\langle I_{i o n}\left(u_{i}^{\varepsilon}-u_{e}^{\varepsilon}, w^{\varepsilon}\right), v_{i}^{\varepsilon}-v_{e}^{\varepsilon}\right\rangle_{\Gamma_{m}^{\varepsilon}}=\varepsilon\left(I_{a p p}^{\varepsilon}, v_{i}^{\varepsilon}-v_{e}^{\varepsilon}\right)_{\Gamma_{m}^{\varepsilon}} .
\end{aligned}
$$


Two-scale convergence theory enables us to relate the 2 -scale limits of $\widetilde{\vec{\nabla}_{\vec{x}} u_{i}^{\varepsilon}}$ and $\widetilde{\vec{\nabla}_{\vec{x}} u_{e}^{\varepsilon}}$ with the 2 -scale limits of the extension of $\left(u_{i}^{\varepsilon}, u_{e}^{\varepsilon}\right)$ defined as

$$
\tilde{u}_{i}^{\varepsilon}=\left\{\begin{array}{cc}
u_{i}^{\varepsilon} & \Omega_{i}^{\varepsilon}, \\
0 & \Omega_{e}^{\varepsilon},
\end{array} \quad \tilde{u}_{e}^{\varepsilon}=\left\{\begin{array}{cc}
0 & \Omega_{i}^{\varepsilon}, \\
u_{e}^{\varepsilon} & \Omega_{e}^{\varepsilon}
\end{array}\right.\right.
$$

Then the a priori estimate (55) allows for the application of the 2-scale convergence theory in a perforated domain as presented in [3]. To do so, we define $H_{\sharp}^{1}(Y)$ - as the completion for the norm $H^{1}(Y)$ of $C_{\sharp}^{\infty}(\bar{Y})$ - the space of infinitely differentiable functions that are periodic of period $Y$ (a similar definition holds if $Y$ is replaced by $Y_{\alpha}$ and $H^{1}(Y)$ is replaced by $\left.L^{2}(Y)\right)$.

Proposition 9. If Assumption 11 holds (i.e. uniform norm-estimate of the data), there exist

$$
u_{\alpha}^{0} \in L^{2}\left((0, T) ; H^{1}(\Omega)\right) \quad \text { and } \quad u_{\alpha}^{1} \in L^{2}\left(\Omega_{T} ; H_{\sharp}^{1}\left(Y_{\alpha}\right)\right),
$$

such that the solution of the bidomain equations given by Theorem 1 satisfies

$$
\left\{\begin{array}{l}
\tilde{u}_{\alpha}^{\varepsilon} \underset{2-\text { scale }}{\longrightarrow} u_{\alpha}^{0}(\vec{x}, t) \chi_{Y_{\alpha}}(\vec{y}), \\
\overrightarrow{\vec{\nabla}}_{\vec{x} u_{\alpha}^{\varepsilon}}^{\longrightarrow} \underset{2-\text { scale }}{\longrightarrow}\left(\vec{\nabla}_{\vec{x}} u_{\alpha}^{0}+\vec{\nabla}_{\vec{y}} u_{\alpha}^{1}\right) \chi_{Y_{\alpha}}(\vec{y}),
\end{array}\right.
$$

where $\chi_{Y_{\alpha}}$ is the characteristic function of $Y_{\alpha}$.

Note that in this proposition, the convergences have to be understood in the sense given in Proposition 7. For regular enough functions, we need to relate the 2 -scale limit on a volume to the 2 -scale limit on surface. This is the object of the proposition given below whose proof is very inspired by [4] (Proposition 2.6) and therefore only the main idea is given.

Proposition 10. Let $\left\{u^{\varepsilon}\right\}$ be a sequence of functions in $L^{2}\left((0, T) ; H^{1}\left(\Omega_{\alpha}^{\varepsilon}\right)\right)$ such that

$$
\int_{0}^{T} \int_{\Omega_{\alpha}^{\varepsilon}}\left|u^{\varepsilon}(\vec{x}, t)\right|^{2}+\left|\vec{\nabla}_{\vec{x}} u^{\varepsilon}(\vec{x}, t)\right|^{2} d \vec{x} d t \leq C,
$$

where $C$ does not depend on $\varepsilon$. Let $\tilde{u}^{\varepsilon}$ denote the extension by zero in $(0, T) \times \Omega$ of $u^{\varepsilon}$. There exists $u^{0} \in L^{2}\left((0, T) ; H^{1}(\Omega)\right)$ such that

$$
\tilde{u}^{\varepsilon}(\vec{x}, t) \underset{2-\text { scale }}{\longrightarrow} u^{0}(\vec{x}, t) \chi_{Y_{\alpha}}(\vec{y})
$$

and for any $\varphi \in C^{0}\left(\overline{\Omega_{T}} ; C_{\sharp}^{0}(\bar{Y})\right)$,

$$
\varepsilon \int_{0}^{T} \int_{\Gamma_{m}^{\varepsilon}} u^{\varepsilon}(\vec{x}, t) \varphi\left(\vec{x}, t, \frac{\vec{x}}{\varepsilon}\right) d \gamma d t \longrightarrow \frac{1}{|Y|} \int_{\Omega_{T}} u^{0}(\vec{x}, t) \int_{\Gamma_{Y}} \varphi(\vec{x}, t, \vec{y}) d \gamma d \vec{x} d t .
$$

Proof. Being given $\varphi \in C^{1}\left(\overline{\Omega_{T}} ; C_{\sharp}^{0}(\bar{Y})\right)$, for each $\vec{x}$ and $t$ (seen here as parameters), we introduce $\psi_{\vec{x}, t}$ a function of $\vec{y}$ periodic in $Y_{i}$ with mean value 0 as the solution of

$$
\left\{\begin{aligned}
\Delta_{\vec{y}} \psi_{\vec{x}, t}=\frac{1}{\left|Y_{i}\right|} \int_{\Gamma_{Y}} \varphi(\vec{x}, t, \vec{y}) d \gamma & \text { in } Y_{i}, \\
\vec{\nabla}_{\vec{y}} \psi_{\vec{x}, t} \cdot \vec{n}_{\Gamma_{Y}}=\varphi(\vec{x}, t, \cdot) & \text { on } \Gamma_{Y},
\end{aligned}\right.
$$

where $\vec{n}_{\Gamma_{Y}}$ is the outward normal of $Y_{i}$. Then the results of the proposition are obtained by first noticing that

$$
\varepsilon \int_{0}^{T} \int_{\Gamma_{m}^{\varepsilon}} u^{\varepsilon}(\vec{x}, t) \varphi\left(\vec{x}, t, \frac{\vec{x}}{\varepsilon}\right) d \gamma \mathrm{d} t=\varepsilon \int_{0}^{T} \int_{\Gamma_{m}^{\varepsilon}} u^{\varepsilon}(\vec{x}, t)\left(\vec{\nabla}_{\vec{y}} \psi_{\vec{x}, t} \cdot \vec{n}_{\Gamma_{Y}}\right)\left(\frac{\vec{x}}{\varepsilon}\right) d \gamma \mathrm{d} t,
$$

then using Green's formulae to recover integral over $\Omega_{i}^{\varepsilon}$ and finally using 2-scale convergence results as in Proposition 7. 


\subsection{The two-scale homogenized limit model}

The next step in deriving the homogenized equations (i.e. setting the equations of the limit terms $u_{\alpha}^{0}$ ) consists in using regular enough test functions in (66) of the form

$$
v_{\alpha}^{\varepsilon}(\vec{x}, t)=v_{\alpha}^{0}(\vec{x}, t)+\varepsilon v_{\alpha}^{1}\left(\vec{x}, t, \frac{\vec{x}}{\varepsilon}\right),
$$

with

$$
\begin{cases}v_{\alpha}^{0} \in C^{1}\left(\overline{\Omega_{T}}\right), & v_{\alpha}^{0}(\vec{x}, T)=0, \\ v_{\alpha}^{1} \in C^{1}\left(\overline{\Omega_{T}} ; C_{\sharp}^{0}\left(\overline{Y_{\alpha}}\right)\right), & v_{\alpha}^{1}(\vec{x}, T, \vec{y})=0 .\end{cases}
$$

The decomposition (71) can be explained a priori since it corresponds to the expected behavior of the limit field, i.e. it should not depend on $\vec{y}$. Doing so, Equation (66) gives, after integration with respect to time,

$$
\begin{aligned}
&\left(\overrightarrow{\vec{\sigma} \varepsilon} \widetilde{\vec{\nabla}_{\vec{x}} u_{i}^{\varepsilon}}, \vec{\nabla}_{\vec{x}} v_{i}^{0}\right.\left.+\vec{\nabla}_{\vec{y}} v_{i}^{1}+\varepsilon \vec{\nabla}_{\vec{x}} v_{i}^{1}\right)_{\Omega_{T}}+\left(\overrightarrow{\vec{\sigma}^{\varepsilon}} \widetilde{\vec{\nabla}_{\vec{x}} u_{e}^{\varepsilon}}, \vec{\nabla}_{\vec{x}} v_{e}^{0}+\vec{\nabla}_{\vec{y}} v_{e}^{1}+\varepsilon \vec{\nabla}_{\vec{x}} v_{e}^{1}\right)_{\Omega_{T}} \\
&-\varepsilon C_{m} \int_{0}^{T}\left\langle u_{i}^{\varepsilon}-u_{e}^{\varepsilon}, \partial_{t}\left(v_{i}^{0}+\varepsilon v_{i}^{1}-v_{e}^{0}-\varepsilon v_{e}^{1}\right)\right\rangle_{\Gamma_{m}^{\varepsilon}} \mathrm{d} t \\
&+\varepsilon \int_{0}^{T}\left\langle I_{i o n}\left(u_{i}^{\varepsilon}-u_{e}^{\varepsilon}, w^{\varepsilon}\right), v_{i}^{0}+\varepsilon v_{i}^{1}-v_{e}^{0}-\varepsilon v_{e}^{1}\right\rangle_{\Gamma_{m}^{\varepsilon}} \mathrm{d} t \\
&=\varepsilon \int_{0}^{T}\left(I_{a p p}^{\varepsilon}, v_{i}^{0}+\varepsilon v_{i}^{1}-v_{e}^{0}-\varepsilon v_{e}^{1}\right)_{\Gamma_{m}^{\varepsilon}} \mathrm{d} t-\varepsilon C_{m}\left(V_{m}^{0, \varepsilon}, v_{i}^{0}+\varepsilon v_{i}^{1}-v_{e}^{0}-\varepsilon v_{e}^{1}\right)_{\Gamma_{m}^{\varepsilon}} .
\end{aligned}
$$

We want to apply the results of the 2-scale convergence. First, we will focus on the volume terms. As explained in [3], the next step is to choose

$$
\vec{\psi}_{\alpha}(\vec{x}, \vec{y}):=\overrightarrow{\vec{\sigma}}(\vec{x}, \vec{y})\left(\vec{\nabla}_{\vec{x}} v_{\alpha}^{0}(\vec{x})+\vec{\nabla}_{\vec{y}} v_{\alpha}^{1}(\vec{x}, \vec{y})\right)
$$

as a test function in the definition of 2-scale convergence. However, Assumption 1 on the diffusion tensor $\overrightarrow{\vec{\sigma}}^{\varepsilon}(\vec{x})$ is not sufficient to have $\vec{\psi}_{\alpha} \in C^{0}\left(\overline{\Omega_{T}} ; L_{\sharp}^{\infty}(Y)\right)^{3}$. This motivates the following additional assumption.

\section{Assumption 12.}

$$
\overrightarrow{\vec{\sigma}}_{\alpha} \in C^{0}\left(\bar{\Omega} ; L_{\#}^{\infty}\left(Y_{\alpha}\right)\right)^{3 \times 3} .
$$

Such an assumption ensures that $\vec{\psi}_{\alpha}$ is an admissible test function (see [3]) and can be considered as a test function in Proposition 7.

The surface terms also need a detailed analysis. Since $I_{a p p}^{\varepsilon}$ is uniformly bounded in $L^{2}\left(\Gamma_{m}^{\varepsilon} \times(0, T)\right)$ (see Assumption 11), we can use Proposition 8 to write that there exists $I_{a p p}^{0} \in L^{2}\left(\Omega_{T} \times \Gamma_{Y}\right)$ such that up to a subsequence,

$$
\varepsilon \int_{0}^{T}\left(I_{a p p}^{\varepsilon}, v_{i}^{0}+\varepsilon v_{i}^{1}-v_{e}^{0}-\varepsilon v_{e}^{1}\right)_{\Gamma_{m}^{\varepsilon}} \mathrm{d} t \longrightarrow \frac{1}{|Y|}\left(\int_{\Gamma_{Y}} I_{a p p}^{0}(\vec{x}, t, \vec{y}) \mathrm{d} \gamma, v_{i}^{0}-v_{e}^{0}\right)_{\Omega_{T}} .
$$

Moreover, since we have assumed that the initial data are also uniformly bounded in the adequate norm (see Assumption 11) and using again the 2-scale convergence theorem on surfaces (Proposition 8), we know that there exists $V_{m}^{0} \in L^{2}\left(\Omega \times \Gamma_{Y}\right)$ such that, up to a subsequence,

$$
\varepsilon C_{m}\left(V_{m}^{0, \varepsilon}, v_{i}^{0}+\varepsilon v_{i}^{1}-v_{e}^{0}-\varepsilon v_{e}^{1}\right)_{\Gamma_{m}^{\varepsilon}} \longrightarrow \frac{C_{m}}{|Y|} \int_{\Omega}\left(\int_{\Gamma_{Y}} V_{m}^{0}(\vec{x}, \vec{y}) d \gamma\right)\left(v_{i}^{0}-v_{e}^{0}\right)(\vec{x}, 0) \mathrm{d} \vec{x} .
$$

In the same way, thanks to Proposition 6, we can show that $I_{i o n}$ satisfies the uniform bound (65) with $p=4 / 3$. We can therefore apply Proposition 8 and find there exists 
$I_{0} \in L^{4 / 3}\left(\Omega_{T} \times \Gamma_{Y}\right)$ such that, up to a subsequence,

$$
\begin{aligned}
\varepsilon \int_{0}^{T}\left\langle I_{\text {ion }}\left(u_{i}^{\varepsilon}-u_{e}^{\varepsilon}, w^{\varepsilon}\right), v_{i}^{0}+\varepsilon v_{i}^{1}-v_{e}^{0}-\varepsilon v_{e}^{1}\right\rangle_{\Gamma_{m}^{\varepsilon}} \mathrm{d} t \\
\longrightarrow \frac{1}{|Y|} \int_{\Omega_{T}}\left(\int_{\Gamma_{Y}} I_{0}(\vec{x}, t, \vec{y}) d \gamma\right)\left(v_{i}^{0}-v_{e}^{0}\right)(\vec{x}, t) \mathrm{d} \vec{x} \mathrm{~d} t .
\end{aligned}
$$

One of the difficulties - which is postponed - is to relate $I_{0}$ with the limits of $u_{\alpha}^{\varepsilon}$ and $w^{\varepsilon}$. To deal with the third term in (72), we also need Proposition 10 and we get

$$
\varepsilon \int_{0}^{T} \int_{\Gamma_{m}^{\varepsilon}} u_{\alpha}^{\varepsilon}(\vec{x}, t) \partial_{t} v_{\alpha}^{\varepsilon}(\vec{x}, t) d \gamma \mathrm{d} t \longrightarrow \frac{\left|\Gamma_{Y}\right|}{|Y|} \int_{\Omega_{T}} u_{\alpha}^{0}(\vec{x}, t) \partial_{t} v_{\alpha}^{0}(\vec{x}, t) \mathrm{d} \vec{x} \mathrm{~d} t .
$$

Using the convergence results obtained above, the weak formulation of the microscopic bidomain equations - as given by (72) - becomes at the limit

$$
\begin{aligned}
\left(\overrightarrow{\vec{\sigma}}_{i}\left(\vec{\nabla}_{\vec{x}} u_{i}^{0}+\vec{\nabla}_{\vec{y}} u_{i}^{1}\right), \vec{\nabla}_{\vec{x}} v_{i}^{0}+\vec{\nabla}_{\vec{y}} v_{i}^{1}\right)_{\Omega_{T} \times Y_{i}}+\left(\overrightarrow{\vec{\sigma}}_{e}\left(\vec{\nabla}_{\vec{x}} u_{e}^{0}+\vec{\nabla}_{\vec{y}} u_{e}^{1}\right), \vec{\nabla}_{\vec{x}} v_{e}^{0}+\vec{\nabla}_{\vec{y}} v_{e}^{1}\right)_{\Omega_{T} \times Y_{e}} \\
-C_{m}\left|\Gamma_{Y}\right|\left(u_{i}^{0}-u_{e}^{0}, \partial_{t}\left(v_{i}^{0}-v_{e}^{0}\right)\right)_{\Omega_{T}}+\left(\int_{\Gamma_{Y}} I_{0} \mathrm{~d} \gamma, v_{i}^{0}-v_{e}^{0}\right)_{\Omega_{T}} \\
=\left(\int_{\Gamma_{Y}} I_{a p p}^{0} d \gamma, v_{i}^{0}-v_{e}^{0}\right)_{\Omega_{T}}-C_{m} \int_{\Omega} \int_{\Gamma_{Y}} V_{m}^{0}(\vec{x}, \vec{y}) d \gamma\left(v_{i}^{0}-v_{e}^{0}\right)(\vec{x}, 0) \mathrm{d} \vec{x} . \quad(77)
\end{aligned}
$$

We now consider the equation on the gating variable. From (45), we deduce that for all

$$
\psi \in C^{1}\left(\overline{\Omega_{T}} ; C_{\sharp}^{0}(\bar{Y})\right) \text { such that } \quad \psi(\vec{x}, T, \vec{y})=0,
$$

we have

$$
\begin{aligned}
-\int_{0}^{T} \int_{\Gamma_{m}^{\varepsilon}} w^{\varepsilon}(\vec{x}, t) \partial_{t} \psi\left(\vec{x}, t, \frac{\vec{x}}{\varepsilon}\right) d \gamma \mathrm{d} t+\int_{0}^{T} \int_{\Gamma_{m}^{\varepsilon}} g\left(V_{m}^{\varepsilon}, w^{\varepsilon}\right) \psi\left(\vec{x}, t, \frac{\vec{x}}{\varepsilon}\right) d \gamma \mathrm{d} t & =-\int_{\Gamma_{m}^{\varepsilon}} w^{0, \varepsilon}(\vec{x}) \psi\left(\vec{x}, 0, \frac{\vec{x}}{\varepsilon}\right) d \gamma
\end{aligned}
$$

Using again the 2-scale convergence of a sequence of $L^{2}$ functions on $\Gamma_{m}^{\varepsilon} \times(0, T)$ (Proposition 8), we find that

$$
-\left(w^{0}, \partial_{t} \psi\right)_{\Omega_{T} \times \Gamma_{Y}}+\left(g_{0}, \psi\right)_{\Omega_{T} \times \Gamma_{Y}}=-\int_{\Omega} \int_{\Gamma_{Y}} w_{0}(\vec{x}, \vec{y}) \psi(\vec{x}, 0, \vec{y}) d \gamma \mathrm{d} \vec{x},
$$

where $w^{0}, g_{0}$ and $w_{0}$ are the 2 -scale limits of $w^{\varepsilon}, g\left(V_{m}^{\varepsilon}, w^{\varepsilon}\right)$ and $w^{0, \varepsilon}$ respectively. These 2 -scale limits are well-defined (up to subsequences) since $w^{\varepsilon}$ is a continuous function in time with value in $L^{2}\left(\Gamma_{m}^{\varepsilon}\right)$ and is uniformly bounded with respect to $\varepsilon$ (Corollary 3 ). The same argument holds for $g$ (Proposition 6).

Finally, one can pass to the 2-scale limit in Equation (48) to recover a condition on the average of $u_{e}^{0}$ and we get the closure equation, for all $\varphi \in C^{0}([0, T])$,

$$
\varepsilon \int_{0}^{T}\left(\int_{\Gamma_{m}^{\varepsilon}} u_{e}^{\varepsilon} d \gamma\right) \varphi \mathrm{d} t=0 \underset{\varepsilon \rightarrow 0}{\longrightarrow} \frac{\left|\Gamma_{Y}\right|}{|Y|} \int_{0}^{T}\left(\int_{\Omega} u_{e}^{0} \mathrm{~d} \vec{x}\right) \varphi \mathrm{d} t=0 .
$$

This implies that we have, for almost all $t \in[0, T]$,

$$
\int_{\Omega} u_{e}^{0} \mathrm{~d} \vec{x}=0
$$


Equations (77), (78) and (79) define some micro-macro equations for the bidomain problem. To close these equations, we need to relate $I_{0}$ and $g_{0}$ to the 2-scale limits of $V_{m}^{\varepsilon}=u_{i}^{\varepsilon}-u_{e}^{\varepsilon}$ and $w^{\varepsilon}$. This is the object of Proposition 11 given in what follows but first, let us mention that is it possible to derive an energy estimate for the micro-macro problem. As mentioned, such energy relations are formally obtained by setting $v_{\alpha}^{0}=e^{-\lambda s} u_{\alpha}^{0}$ and $v_{\alpha}^{1}=e^{-\lambda s} u_{\alpha}^{1}$ in Equation (77) and $\psi=e^{-\lambda s} w^{0}$ in Equation (78). The following energy relation can be proven, for all $\lambda>0$ and $\mu>0$,

$$
\begin{aligned}
& \mathcal{E}_{\lambda, \mu}^{0}\left(u_{i}^{0}, u_{i}^{1}, u_{e}^{0}, u_{e}^{1}, w^{0}, t\right)-\mathcal{E}_{\lambda, \mu}^{0}\left(u_{i}^{0}, u_{i}^{1}, u_{e}^{0}, u_{e}^{1}, w^{0}, 0\right) \\
&+\int_{0}^{t} e^{-\lambda s}\left[\left(\int_{\Gamma_{Y}} I_{0} \mathrm{~d} \gamma, v_{i}^{0}-v_{e}^{0}\right)_{\Omega}+\frac{\lambda C_{m}\left|\Gamma_{Y}\right|}{2}\left\|\left(u_{i}^{0}-u_{e}^{0}\right)(t)\right\|_{L^{2}(\Omega)}^{2}\right] \mathrm{d} s \\
&+\int_{0}^{t} e^{-\lambda s}\left[\mu\left(g_{0}, w^{0}\right)_{L^{2}\left(\Omega \times \Gamma_{Y}\right)}+\lambda \frac{\mu}{2}\left\|w^{0}\right\|_{L^{2}\left(\Omega \times \Gamma_{Y}\right)}^{2}\right] \mathrm{d} s \\
&=\int_{0}^{t} e^{-\lambda s}\left(\int_{\Gamma_{Y}} I_{a p p}^{0} \mathrm{~d} \gamma, u_{i}^{0}-u_{e}^{0}\right)_{\Omega} \mathrm{d} s,
\end{aligned}
$$

where the term $\mathcal{E}_{\lambda, \mu}^{0}$ is the energy associated with the system and is defined by

$$
\begin{array}{r}
\mathcal{E}_{\lambda, \mu}^{0}\left(u_{i}^{0}, u_{i}^{1}, u_{e}^{0}, u_{e}^{1}, w^{0}, t\right)=\frac{C_{m}\left|\Gamma_{Y}\right|}{2} e^{-\lambda t}\left\|\left(u_{i}^{0}-u_{e}^{0}\right)(t)\right\|_{L^{2}(\Omega)}^{2}+\frac{\mu}{2} e^{-\lambda t}\left\|w^{0}(t)\right\|_{L^{2}\left(\Omega \times \Gamma_{Y}\right)}^{2} \\
+\sum_{\alpha \in\{i, e\}} \int_{0}^{t} e^{-\lambda s}\left(\overrightarrow{\vec{\sigma}}_{\alpha}\left(\vec{\nabla}_{\vec{x}} u_{\alpha}^{0}+\vec{\nabla}_{\vec{y}} u_{\alpha}^{1}\right), \vec{\nabla}_{\vec{x}} u_{\alpha}^{0}+\vec{\nabla}_{\vec{y}} u_{\alpha}^{1}\right)_{\Omega \times Y_{\alpha}} \mathrm{d} s .
\end{array}
$$

As previously mentioned, the following proposition relates $I_{0}$ and $g_{0}$ to the 2-scale limits of $V_{m}^{\varepsilon}=u_{i}^{\varepsilon}-u_{e}^{\varepsilon}$ and $w^{\varepsilon}$, respectively.

Proposition 11. We assume that Assumptions 8 and 12 are satisfied and that the source term and the initial data are given by,

$$
I_{a p p}^{\varepsilon}(\vec{x}, t)=I_{a p p}(\vec{x}, t), \quad V_{m}^{0, \varepsilon}(\vec{x})=V_{m}^{0}(\vec{x}), \quad w^{0, \varepsilon}(\vec{x})=w_{0}(\vec{x}), \forall t \in[0, T], \forall \vec{x} \in \Gamma_{m}^{\varepsilon},
$$

with

$$
I_{a p p} \in C^{0}\left(\overline{\Omega_{T}}\right), \quad V_{m}^{0} \in C^{0}\left(\overline{\Omega_{T}}\right), \quad w_{0} \in C^{0}\left(\overline{\Omega_{T}}\right) .
$$

Let $\left(u_{i}^{\varepsilon}, u_{e}^{\varepsilon}, w^{\varepsilon}\right)$ be a solution of equations (45)-(48) given by Theorem 1 and let $u_{\alpha}^{0}, w^{0}, I_{0}$ and $g_{0}$, the 2-scale limits of $u_{\alpha}^{\varepsilon}, w^{\varepsilon}, I_{i o n}\left(u_{i}^{\varepsilon}-u_{e}^{\varepsilon}, w^{\varepsilon}\right)$ and $g\left(u_{i}^{\varepsilon}-u_{e}^{\varepsilon}, w^{\varepsilon}\right)$ respectively then we have,

$$
\left\{\begin{array}{l}
I_{0}=I_{i o n}\left(u_{i}^{0}-u_{e}^{0}, w^{0}\right) \\
g_{0}=g\left(u_{i}^{0}-u_{e}^{0}, w^{0}\right) .
\end{array}\right.
$$

Proof. This result is not a straightforward consequence of the $u_{\alpha}^{\varepsilon}$ and $w^{\varepsilon}$ estimates. We adapt here the technique used in [3] for the 2-scale analysis of non-linear problems. Thanks to Assumption 8, for $\lambda>0$ large enough (but independent of $\varepsilon$ ) and $\mu>0$ given, for all regular enough test functions $\varphi_{i}^{\varepsilon}, \varphi_{e}^{\varepsilon}$ and $\psi^{\varepsilon}$, we have

$$
\begin{aligned}
& \mathcal{E}_{\lambda, \mu}\left(u_{i}^{\varepsilon}-\varphi_{i}^{\varepsilon}, u_{e}^{\varepsilon}-\varphi_{e}^{\varepsilon}, w^{\varepsilon}-\psi^{\varepsilon}, T\right) \\
& +\varepsilon \int_{0}^{T} e^{-\lambda t}\left[\left\langle I_{i o n}\left(V_{m}^{\varepsilon}, w^{\varepsilon}\right)-I_{i o n}\left(\nu_{m}^{\varepsilon}, \psi^{\varepsilon}\right), V_{m}^{\varepsilon}-\nu_{m}^{\varepsilon}\right\rangle_{\Gamma_{m}^{\varepsilon}}+\frac{\lambda C_{m}}{2}\left\|V_{m}^{\varepsilon}-\nu_{m}^{\varepsilon}\right\|_{L^{2}\left(\Gamma_{m}^{\varepsilon}\right)}^{2}\right] \mathrm{d} t \\
& +\varepsilon \int_{0}^{T} e^{-\lambda t}\left[\mu\left(g\left(V_{m}^{\varepsilon}, w^{\varepsilon}\right)-g\left(\nu_{m}^{\varepsilon}, \psi^{\varepsilon}\right), w^{\varepsilon}-\psi^{\varepsilon}\right)_{\Gamma_{m}^{\varepsilon}}+\lambda \frac{\mu}{2}\left\|w^{\varepsilon}-\psi^{\varepsilon}\right\|_{L^{2}\left(\Gamma_{m}^{\varepsilon}\right)}^{2}\right] \mathrm{d} t \geq 0,
\end{aligned}
$$

where $\nu_{m}^{\varepsilon}=\varphi_{i}^{\varepsilon}-\varphi_{e}^{\varepsilon}$ on $\Gamma_{m}^{\varepsilon}$ and where the energy functional $\mathcal{E}_{\lambda, \mu}$ is defined in Equation (52). The idea is to use the energy relation (51) - which is satisfied by the solution $\left(u_{i}^{\varepsilon}, u_{e}^{\varepsilon}, w^{\varepsilon}\right)-$ 
to simplify the previous equation. Doing so, we introduce a term corresponding to the data

$$
d^{\varepsilon}(T):=\varepsilon \int_{0}^{T} e^{-\lambda t}\left(I_{a p p}, V_{m}^{\varepsilon}\right)_{\Gamma_{m}^{\varepsilon}} \mathrm{d} t+\varepsilon \frac{C_{m}}{2}\left\|V_{m}^{0}\right\|_{L^{2}\left(\Gamma_{m}^{\varepsilon}\right)}^{2}+\varepsilon \frac{\mu}{2}\left\|w_{0}\right\|_{L^{2}\left(\Gamma_{m}^{\varepsilon}\right)}^{2} .
$$

Substituting (51) into (83), we obtain the inequality

$$
0 \leq d^{\varepsilon}(T)-2 e^{\varepsilon}(T)+\mathcal{E}_{\lambda, \mu}\left(\varphi_{i}^{\varepsilon}, \varphi_{e}^{\varepsilon}, \psi^{\varepsilon}, T\right)+i^{\varepsilon}(T)+\mu g^{\varepsilon}(T),
$$

with

$$
\begin{gathered}
e^{\varepsilon}(T)=\varepsilon \frac{C_{m}}{2} e^{-\lambda T}\left(V_{m}^{\varepsilon}, \nu_{m}^{\varepsilon}\right)_{\Gamma_{m}^{\varepsilon}}+\varepsilon \frac{\mu}{2} e^{-\lambda T}\left(w^{\varepsilon}, \psi^{\varepsilon}\right)_{\Gamma_{m}^{\varepsilon}} \\
+\int_{0}^{T} e^{-\lambda t}\left(\overrightarrow{\vec{\sigma}}_{i} \vec{\nabla}_{\vec{x}} u_{i}^{\varepsilon}, \vec{\nabla}_{\vec{x}} \varphi_{i}^{\varepsilon}\right)_{\Omega_{i}^{\varepsilon}} \mathrm{d} t+\int_{0}^{T} e^{-\lambda t}\left(\overrightarrow{\vec{\sigma}}_{e} \vec{\nabla}_{\vec{x}} u_{e}^{\varepsilon}, \vec{\nabla}_{\vec{x}} \varphi_{e}^{\varepsilon}\right)_{\Omega_{e}^{\varepsilon}} \mathrm{d} t, \\
i^{\varepsilon}(T)=\int_{0}^{T} e^{-\lambda t}\left[-\varepsilon\left\langle I_{i o n}\left(V_{m}^{\varepsilon}, w^{\varepsilon}\right), \nu_{m}^{\varepsilon}\right\rangle_{\Gamma_{m}^{\varepsilon}}-\varepsilon\left\langle I_{i o n}\left(\nu_{m}^{\varepsilon}, \psi^{\varepsilon}\right), V_{m}^{\varepsilon}-\nu_{m}^{\varepsilon}\right\rangle_{\Gamma_{m}^{\varepsilon}}\right. \\
\left.-\varepsilon \lambda C_{m}\left(V_{m}^{\varepsilon}, \nu_{m}^{\varepsilon}\right)_{L^{2}\left(\Gamma_{m}^{\varepsilon}\right)}^{2}+\varepsilon \frac{\lambda C_{m}}{2}\left\|\nu_{m}^{\varepsilon}\right\|_{L^{2}\left(\Gamma_{m}^{\varepsilon}\right)}^{2}\right] \mathrm{d} t,
\end{gathered}
$$

and

$$
\begin{gathered}
g^{\varepsilon}=\int_{0}^{T} e^{-\lambda t}\left[-\varepsilon\left(g\left(V_{m}^{\varepsilon}, w^{\varepsilon}\right), \nu_{m}^{\varepsilon}\right)_{\Gamma_{m}^{\varepsilon}}-\varepsilon\left(g\left(\nu_{m}^{\varepsilon}, \psi^{\varepsilon}\right), w^{\varepsilon}-\psi^{\varepsilon}\right)_{\Gamma_{m}^{\varepsilon}}\right. \\
\left.-\varepsilon \lambda\left(w^{\varepsilon}, \psi^{\varepsilon}\right)_{L^{2}\left(\Gamma_{m}^{\varepsilon}\right)}^{2}+\varepsilon \frac{\lambda}{2}\left\|\psi^{\varepsilon}\right\|_{L^{2}\left(\Gamma_{m}^{\varepsilon}\right)}^{2}\right] \mathrm{d} t .
\end{gathered}
$$

Now, we set, for any given positive real scalar $\tau$,

$$
\left\{\begin{array}{l}
\varphi_{\alpha}^{\varepsilon}(\vec{x}, t)=\varphi_{\alpha}^{0}(\vec{x}, t)+\varepsilon \varphi_{\alpha}^{1}(\vec{x}, t, \vec{x} / \varepsilon)+\tau \varphi_{\alpha}(\vec{x}, t), \\
\psi^{\varepsilon}(\vec{x}, t)=\psi^{0}(\vec{x}, t, \vec{x} / \varepsilon)+\tau \psi(\vec{x}, t, \vec{x} / \varepsilon)
\end{array}\right.
$$

where

$$
\left(\varphi_{\alpha}^{0}, \varphi_{\alpha}\right) \in C^{1}\left(\overline{\Omega_{T}}\right)^{2}, \quad \varphi_{\alpha}^{1} \in C^{1}\left(\overline{\Omega_{T}} ; C_{\sharp}^{1}(\bar{Y})\right), \quad\left(\psi_{0}, \psi\right) \in C^{0}\left(\overline{\Omega_{T}} ; C_{\sharp}^{0}(\bar{Y})\right)^{2} .
$$

By construction, $\varphi_{i}^{\varepsilon}$ and $\varphi_{e}^{\varepsilon}$ are test functions that allow us to use the 2-scale convergence (see Propositions 7 and 8). The same remark is true for $I_{a p p}, V_{m}^{0}$ and $w^{0}$ by assumption. Moreover, we need the following results concerning 2-scale convergence of test functions (see [3])

$$
\chi_{Y_{\alpha}}\left(\frac{\vec{x}}{\varepsilon}\right) \varphi_{\alpha}^{\varepsilon} \underset{2-\text { scale }}{\longrightarrow} \chi_{Y_{\alpha}}(\vec{y})\left(\varphi_{\alpha}^{0}+\tau \varphi_{\alpha}\right)
$$

and

$$
\chi_{Y_{\alpha}}\left(\frac{\vec{x}}{\varepsilon}\right) \vec{\nabla}_{\vec{x}} \varphi_{\alpha}^{\varepsilon} \underset{2-\text { scale }}{\longrightarrow} \chi_{Y_{\alpha}}(\vec{y})\left(\vec{\nabla}_{\vec{x}} \varphi_{\alpha}^{0}+\vec{\nabla}_{\vec{y}} \varphi_{\alpha}^{1}+\tau \vec{\nabla}_{\vec{x}} \varphi_{\alpha}\right),
$$

where the convergence has to be understood in the sense given by Proposition 7. Moreover,

$$
\nu_{m}^{\varepsilon} \underset{2-\text { scale }}{\longrightarrow} \Phi^{0}+\tau \Phi, \quad \psi^{\varepsilon} \underset{2-\text { scale }}{\longrightarrow} \psi^{0}+\tau \psi
$$

where

$$
\Phi^{0}=\varphi_{i}^{0}-\varphi_{e}^{0} \quad \text { and } \quad \Phi=\varphi_{i}-\varphi_{e}
$$


and where the convergence has to be understood in the sense given by Proposition 8 . To study the limit of (84) when $\varepsilon$ tends to 0 , we treat each terms separately. We first have $\lim _{\varepsilon \rightarrow 0} d^{\varepsilon}=d^{0}$ with

$$
d^{0}(T)=\left|\Gamma_{Y}\right| \int_{0}^{T} e^{-\lambda t}\left(I_{a p p}, V^{0}\right)_{\Omega} \mathrm{d} t+\frac{\left|\Gamma_{Y}\right| C_{m}}{2}\left\|V_{m}^{0}\right\|_{L^{2}(\Omega)}^{2}+\frac{\left|\Gamma_{Y}\right| \mu}{2}\left\|w_{0}\right\|_{L^{2}(\Omega)}^{2} .
$$

In the same way, one can pass to the limit $\varepsilon \rightarrow 0$ in $e^{\varepsilon}(t)$ and we denote by $e^{0}(t)$ this limit. It is given by

$$
\begin{aligned}
e^{0}(T)=\frac{C_{m}\left|\Gamma_{Y}\right|}{2} e^{-\lambda t}\left(u_{i}^{0}-u_{e}^{0}, \Phi^{0}+\tau \Phi\right)_{\Omega}+\frac{\mu}{2} e^{-\lambda t}\left(w^{0}, \psi^{0}+\tau \psi\right)_{\Omega \times \Gamma_{Y}} \\
+\sum_{\alpha \in\{i, e\}} \int_{0}^{T} e^{-\lambda s}\left(\overrightarrow{\vec{\sigma}}_{\alpha}\left(\vec{\nabla}_{\vec{x}} u_{\alpha}^{0}+\vec{\nabla}_{\vec{y}} u_{\alpha}^{1}\right), \vec{\nabla}_{\vec{x}} \varphi_{\alpha}^{0}+\vec{\nabla}_{\vec{y}} \varphi_{\alpha}^{1}+\tau \vec{\nabla}_{\vec{x}} \varphi_{\alpha}\right)_{\Omega \times Y_{\alpha}} \mathrm{d} t .
\end{aligned}
$$

We also get

$$
e_{\lambda, \mu}^{0}(T):=\lim _{\varepsilon \rightarrow 0} \mathcal{E}_{\lambda, \mu}\left(\varphi_{i}^{\varepsilon}, \varphi_{e}^{\varepsilon}, \psi^{\varepsilon}, T\right)=\mathcal{E}_{\lambda, \mu}^{0}\left(\varphi_{i}^{0}+\tau \varphi_{i}, \varphi_{i}^{1}, \varphi_{e}^{0}+\tau \varphi_{e}, \varphi_{e}^{1}, \psi^{0}+\tau \psi, T\right),
$$

where $\mathcal{E}_{\lambda, \mu}^{0}$ is the energy of the limit 2-scale problem defined in Equation (82). Note that to pass to the limit in the microscopic energy, we have used the strong 2 -scale convergence of test functions. Indeed using [4] (Lemma 2.4), we have

$$
\lim _{\varepsilon \rightarrow 0} \varepsilon\left\|\psi^{\varepsilon}\right\|_{L^{2}\left(\Gamma_{m}^{\varepsilon}\right)}^{2}=\left\|\psi^{0}+\tau \psi\right\|_{L^{2}\left(\Omega \times \Gamma_{Y}\right)}^{2} .
$$

To pass to the limit in the terms $i^{\varepsilon}$ and $g^{\varepsilon}$, we need to study the convergence of $I_{i o n}\left(\nu_{m}^{\varepsilon}, \psi^{\varepsilon}\right)$ and $g\left(\nu_{m}^{\varepsilon}, \psi^{\varepsilon}\right)$ respectively. Since the function $I_{i o n}$ is continuous (Assumption 4), as well as $\left(\Phi^{0}, \Phi, \psi^{0}, \psi\right)$, one can see that $I_{i o n}\left(\Phi^{0}+\tau \Phi, \psi^{0}+\tau \psi\right)$ is an adequate test function in the sense of Proposition 8, i.e.

$$
I_{\text {ion }}\left(\Phi^{0}+\tau \Phi, \psi^{0}+\tau \psi\right) \in C^{0}\left(\overline{\Omega_{T}} ; C_{\sharp}^{0}(\bar{Y})\right) .
$$

Moreover, we have,

$$
\lim _{\varepsilon \rightarrow 0}\left\|I_{i o n}\left(\nu_{m}^{\varepsilon}, \psi^{\varepsilon}\right)-I_{i o n}\left(\Phi^{0}+\tau \Phi, \psi^{0}+\tau \psi\right)\right\|_{L^{\infty}\left(\Omega_{T} \times \Gamma_{Y}\right)}=0,
$$

and therefore

$$
\begin{aligned}
& \lim _{\varepsilon \rightarrow 0} \varepsilon \int_{0}^{T} e^{-\lambda t}\left\langle I_{\text {ion }}\left(\nu_{m}^{\varepsilon}, \psi^{\varepsilon}\right), V_{m}^{\varepsilon}-\nu_{m}^{\varepsilon}\right\rangle_{\Gamma_{m}^{\varepsilon}} \mathrm{d} t \\
&=\int_{0}^{T} e^{-\lambda t}\left(I_{\text {ion }}\left(\Phi^{0}+\tau \Phi, \psi^{0}+\tau \psi\right), V_{m}^{0}-\Phi^{0}-\tau \Phi\right)_{\Omega \times \Gamma_{Y}} \mathrm{~d} t .
\end{aligned}
$$

Using the results above, one can show that

$$
\begin{aligned}
i^{0}(T):=\lim _{\varepsilon \rightarrow 0} i^{\varepsilon}(T)= & \int_{0}^{T} e^{-\lambda t}\left[-\left(I_{0}, \Phi^{0}+\tau \Phi\right)_{\Omega \times \Gamma_{Y}}\right. \\
- & \left(I_{\text {ion }}\left(\Phi^{0}+\tau \Phi, \psi^{0}+\tau \psi\right), V_{m}^{0}-\Phi^{0}-\tau \Phi\right)_{\Omega \times \Gamma_{Y}} \\
& \left.-\lambda\left|\Gamma_{Y}\right| C_{m}\left(V_{m}^{0}, \Phi^{0}+\tau \Phi\right)_{L^{2}(\Omega)}^{2}+\frac{\lambda\left|\Gamma_{Y}\right| C_{m}}{2}\left\|\Phi^{0}+\tau \Phi\right\|_{L^{2}(\Omega)}^{2}\right] \mathrm{d} t .
\end{aligned}
$$

Similar results can be deduced in order to compute the limit of $g_{\varepsilon}$ which we denote $g_{0}$. Collecting all the convergence results mentioned above, Inequality (84) becomes

$$
0 \leq d^{0}(T)-2 e^{0}(T)+e_{\lambda, \mu}^{0}(T)+i^{0}(T)+\mu g^{0}(T)
$$


Since this inequality is true for all $\varphi_{\alpha}^{0}, \varphi_{\alpha}^{1}$ and $\psi^{0}$, it is true for each element of the sequences $\left\{\varphi_{\alpha, n}^{0}\right\},\left\{\varphi_{\alpha, n}^{1}\right\}$ and $\left\{\psi_{n}^{0}\right\}$ that satisfy, by density of regular functions

$$
\lim _{n \rightarrow+\infty} \sum_{\alpha \in\{i, e\}}\left(\left\|\varphi_{\alpha, n}^{0}-u_{\alpha}^{0}\right\|_{L^{2}\left((0, T) ; H^{1}(\Omega)\right)}+\left\|\varphi_{\alpha, n}^{1}-u_{\alpha}^{1}\right\|_{L^{2}\left(\Omega_{T} ; H^{1}\left(Y_{\alpha}\right)\right)}\right)=0
$$

and

$$
\lim _{n \rightarrow+\infty}\left\|\psi_{n}^{0}-w_{0}\right\|_{L^{2}\left(\Omega_{T} \times \Gamma_{Y}\right)}=0 .
$$

From the continuity requirement (Assumption 4) and the growth conditions (28) - (29), $I_{i o n}$ and $g$ can be seen as weak continuous applications. Such results are a consequence of a variant of Lemma 1.3 of [33] (the same proof can be followed) that can be stated as follows: (for conciseness, we set $\mathcal{D}=\Omega \times \Gamma_{Y}$ )

Let $\left\{I_{n}\right\}$, a uniformly bounded sequence and $I$, a function in $L^{2}\left((0, T), L^{p}(\mathcal{D})\right)$ with $1<p<$ $+\infty$. Assume that the sequence $\left\{I_{n}\right\}$ converges almost everywhere to $I$ in $(0, T) \times \mathcal{D}$, then $I_{n}$ converges weakly towards $I$ in $L^{2}\left((0, T), L^{p}(\mathcal{D})\right)$.

Using the result above one can pass to the limit in Inequality (85), it shows that this inequality is true after the following formal substitutions, $\varphi_{\alpha}^{0}=u_{\alpha}^{0}, \varphi_{\alpha}^{1}=u_{\alpha}^{1}$ and $\psi^{0}=w^{0}$. Using the energy identity given in Equation (81), one can simplify Inequality (85) and we obtain

$$
\begin{aligned}
0 \leq \tau \int_{0}^{T} e^{-\lambda t}\left(I _ { i o n } \left(V_{m}^{0}\right.\right. & \left.\left.+\tau \Phi, w^{0}+\tau \psi\right)-I_{0}, \Phi\right)_{\Omega \times \Gamma_{Y}} \mathrm{~d} t \\
& +\tau \int_{0}^{T} e^{-\lambda t}\left(g\left(V_{m}^{0}+\tau \Phi, w^{0}+\tau \psi\right)-g_{0}, \psi\right)_{\Omega \times \Gamma_{Y}} \mathrm{~d} t+O\left(\tau^{2}\right) .
\end{aligned}
$$

Dividing by $\tau$ and then letting $\tau$ tends to 0 , we find that for all continuous functions $\Phi$ and $\psi$

$$
0 \leq \int_{0}^{T} e^{-\lambda t}\left(I_{i o n}\left(V_{m}^{0}, w^{0}\right)-I_{0}, \Phi\right)_{\Omega \times \Gamma_{Y}} \mathrm{~d} t+\int_{0}^{T} e^{-\lambda t}\left(g\left(V_{m}^{0}, w^{0}\right)-g_{0}, \psi\right)_{\Omega \times \Gamma_{Y}} \mathrm{~d} t
$$

which gives the result of the proposition.

\subsection{The macroscopic bidomain equations}

The obtained model combines a priori the micro- and the macroscopic scales. However, we will show below that we can decouple these two scales by explicitly determining the correction terms $u_{i}^{1}$ and $u_{e}^{1}$. This determination appears through the analysis of canonical problems which are set in the reference periodic cells $Y_{i}$ and $Y_{e}$. First, we choose $v_{i}^{0}=v_{e}^{0}=$ $v_{e}^{1}=0$ and $(77)$ becomes

$$
\left(\overrightarrow{\vec{\sigma}}_{i}\left(\vec{\nabla}_{\vec{x}} u_{i}^{0}+\vec{\nabla}_{\vec{y}} u_{i}^{1}\right), \vec{\nabla}_{\vec{y}} v_{i}^{1}\right)_{\Omega_{T} \times Y_{i}}=0 .
$$

One can observe that such a problem corresponds to the classical cell problem, see [3, 9]. It can be shown that $u_{i}^{1}$ are defined up to a function $\tilde{u}_{i}^{1} \in L^{2}\left(\Omega_{T}\right)$ and can be decomposed as follows

$$
u_{i}^{1}(\vec{x}, \vec{y}, t)=\sum_{j=1}^{3} \mathcal{X}_{i}^{j}(\vec{y}) \vec{\nabla}_{\vec{x}} u_{i}^{0}(\vec{x}, t) \cdot \vec{e}^{j}+\tilde{u}_{i}^{1}(\vec{x}, t),
$$

where the canonical functions $\mathcal{X}_{i}^{j}, j=1 . .3$ belong to $H_{\sharp}^{1}\left(Y_{i}\right)$ and are uniquely defined by the following variational formulation

$$
\left\{\begin{array}{l}
\left(\overrightarrow{\vec{\sigma}}_{i}\left(\vec{e}^{j}+\vec{\nabla}_{\vec{y}} \mathcal{X}_{i}^{j}\right), \vec{\nabla}_{\vec{y}} \psi\right)_{Y_{i}}=0, \forall \psi \in H_{\sharp}^{1}\left(Y_{i}\right), \\
\int_{Y_{i}} \mathcal{X}_{i}^{j} \mathrm{~d} \vec{y}=0 .
\end{array}\right.
$$


From the canonical functions, one can define the associated effective medium tensor $\overrightarrow{\vec{T}}_{i}$ as follows

$$
\left(\overrightarrow{\vec{T}}_{i}\right)_{j, k}=\int_{Y_{i}} \overrightarrow{\vec{\sigma}}_{i}\left(\vec{\nabla}_{\vec{y}} \mathcal{X}_{i}^{j}+\vec{e}^{j}\right) \cdot\left(\vec{\nabla}_{\vec{y}} \mathcal{X}_{i}^{k}+\vec{e}^{k}\right) \mathrm{d} \vec{y} .
$$

We use exactly the same method in order to define and decouple the cell problem in the extracellular domain $Y_{e}$ and to define the effective medium $\overrightarrow{\vec{T}}_{e}$.

The macroscopic equations are obtained by taking $v_{i}^{1}=v_{e}^{1}=v_{e}^{0}=0$ in (77) and we get

$$
\begin{aligned}
\left(\overrightarrow{\vec{\sigma}}_{i}\left(\vec{\nabla}_{\vec{x}} u_{i}^{0}+\vec{\nabla}_{\vec{y}} u_{i}^{1}\right), \vec{\nabla}_{\vec{x}} v_{i}^{0}\right)_{\Omega_{T} \times Y_{i}}-C_{m}\left|\Gamma_{Y}\right|\left(u_{i}^{0}-u_{e}^{0}, \partial_{t} v_{i}^{0}\right)_{\Omega_{T}} & \\
+\left(\int_{\Gamma_{Y}} I_{i o n}\left(u_{i}^{0}-u_{e}^{0}, w^{0}\right) \mathrm{d} \gamma, v_{i}^{0}\right)_{\Omega_{T}} & =\left|\Gamma_{Y}\right|\left(I_{a p p}, v_{i}^{0}\right)_{\Omega_{T}} \\
& -C_{m}\left|\Gamma_{Y}\right| \int_{\Omega} V_{m}^{0}(\vec{x}) v_{i}^{0}(\vec{x}, 0) \mathrm{d} \vec{x} .
\end{aligned}
$$
obtain

Using the decomposition of $\vec{\nabla}_{\vec{y}} u_{i}^{1}=\sum_{j=1}^{3} \vec{\nabla}_{\vec{y}} \mathcal{X}_{i}^{j} \partial_{x^{j}} u_{i}^{0}$ and $\vec{\nabla}_{\vec{x}} u_{i}^{0}=\sum_{j=1}^{3} \partial_{x^{j}} u_{i}^{0} \vec{e}^{j}$, we

$$
\begin{aligned}
\left(\overrightarrow{\vec{\sigma}}_{i}\left(\vec{\nabla}_{\vec{x}} u_{i}^{0}+\vec{\nabla}_{\vec{y}} u_{i}^{1}\right), \vec{\nabla}_{\vec{x}} v_{i}^{0}\right)_{\Omega_{T} \times Y_{i}}=\left(\overrightarrow{\vec{\sigma}}_{i} \sum_{j=1}^{3}\left(\vec{e}^{j}+\vec{\nabla}_{\vec{y}} \mathcal{X}_{i}^{j}\right) \partial_{x^{j}} u_{i}^{0}, \vec{\nabla}_{\vec{x}} v_{i}^{0}\right)_{\Omega_{T} \times Y_{i}} & =\left(\overrightarrow{\vec{T}}_{i} \vec{\nabla}_{\vec{x}} u_{i}^{0}, \vec{\nabla}_{\vec{x}} v_{i}^{0}\right)_{\Omega_{T}} .
\end{aligned}
$$

This equality allows us to simplify Equation (90). In the same way, for the extra-cellular part, we get

$$
\begin{array}{r}
\left(\overrightarrow{\vec{T}}_{e} \vec{\nabla}_{\vec{x}} u_{e}^{0}, \vec{\nabla}_{\vec{x}} v_{e}^{0}\right)_{\Omega_{T}}+C_{m}\left|\Gamma_{Y}\right|\left(u_{i}^{0}-u_{e}^{0}, \partial_{t} v_{e}^{0}\right)_{\Omega_{T}}-\left(\int_{\Gamma_{Y}} I_{i o n}\left(u_{i}^{0}-u_{e}^{0}, w^{0}\right) \mathrm{d} \gamma, v_{e}^{0}\right)_{\Omega_{T}} \\
=-\left|\Gamma_{Y}\right|\left(I_{a p p}, v_{e}^{0}\right)_{\Omega_{T}}+C_{m}\left|\Gamma_{Y}\right| \int_{\Omega} V_{m}^{0}(\vec{x}) v_{e}^{0}(\vec{x}, 0) \mathrm{d} \vec{x} .
\end{array}
$$

Note that Equations (90) and (91) are not yet satisfactory because $I_{i o n}\left(u_{i}^{0}-u_{e}^{0}, w^{0}\right)$ may depend on $\vec{y}$ since $w^{0}$ is a priori a function of $\vec{y}$. However, we have assumed that the initial data do not depend on $\varepsilon$ therefore, we get

$$
-\left(w^{0}, \partial_{t} \psi\right)_{\Omega_{T} \times \Gamma_{Y}}+\left(g\left(u_{i}^{0}-u_{e}^{0}, w^{0}\right), \psi\right)_{\Omega_{T} \times \Gamma_{Y}}=-\int_{\Omega} w_{0}(\vec{x}) \int_{\Gamma_{Y}} \psi(\vec{x}, 0, \vec{y}) d \gamma \mathrm{d} \vec{x},
$$

which is the weak formulation of the following problem

$$
\begin{cases}\frac{\partial w^{0}}{\partial t}+g\left(u_{i}^{0}-u_{e}^{0}, w^{0}\right)=0 & \Omega \times(0, T) \times \Gamma_{Y}, \\ w^{0}(\vec{x}, 0, \vec{y})=w_{0}(\vec{x}) & \Omega \times \Gamma_{Y} .\end{cases}
$$

Since the non-linear term $g$ is not varying at the micro scale and since $\left(u_{i}^{0}-u_{e}^{0}\right)$ does not depend on $\vec{y}$, it can be proven, using Assumption 8, that the solution $w^{0}$ of (92) is unique for all $\vec{y} \in \Gamma_{Y}$ hence it is independent of the variable $\vec{y}$. As a consequence, we have

$$
\frac{1}{|Y|} \int_{\Gamma_{Y}} I_{i o n}\left(u_{i}^{0}-u_{e}^{0}, w^{0}\right)=A_{m} I_{i o n}\left(u_{i}^{0}-u_{e}^{0}, w^{0}\right)
$$

where $A_{m}=\left|\Gamma_{Y}\right| /|Y|$ is the ratio of membrane area per unit volume. Now observe that 
Equations (90) and (91) are the weak forms of the following set of equations

$$
\begin{cases}-\vec{\nabla}_{\vec{x}} \cdot \frac{\overrightarrow{\vec{T}}_{i}}{|Y|} \vec{\nabla}_{\vec{x}} u_{i}^{0}+A_{m} C_{m} \frac{\partial\left(u_{i}^{0}-u_{e}^{0}\right)}{\partial t}+A_{m} I_{i o n}\left(u_{i}^{0}-u_{e}^{0}, w^{0}\right)=A_{m} I_{a p p} & \Omega \times(0, T), \\ -\vec{\nabla}_{\vec{x}} \cdot \frac{\overrightarrow{\vec{T}}_{e}}{|Y|} \vec{\nabla}_{\vec{x}} u_{e}^{0}-A_{m} C_{m} \frac{\partial\left(u_{i}^{0}-u_{e}^{0}\right)}{\partial t}-A_{m} I_{i o n}\left(u_{i}^{0}-u_{e}^{0}, w^{0}\right)=-A_{m} I_{a p p} & \Omega \times(0, T), \\ \left(\overrightarrow{\vec{T}}_{i} \cdot \vec{\nabla}_{\vec{x}} u_{i}\right) \cdot \vec{n}=0 & \partial \Omega \times(0, T), \\ \left(\overrightarrow{\vec{T}}_{e} \cdot \vec{\nabla}_{\vec{x}} u_{e}\right) \cdot \vec{n}=0 & \partial \Omega \times(0, T), \\ \left(u_{i}^{0}-u_{e}^{0}\right)(\vec{x}, 0)=V_{m}^{0}(\vec{x}) & \Omega .\end{cases}
$$

System (93)-(92) corresponds to the sought macro-scale equations. Finally, note that we close the problem by recalling Equation (80)

$$
\int_{\Omega} u_{e}^{0} \mathrm{~d} \vec{x}=0 .
$$

Since the analysis has already been done for the microscopic bidomain model, we can infer - up to a subsequence - the existence of a solution of System (92)-(93). The proposed model is a generalization of the very classical macroscopic bidomain model in which constant electric conductivities are considered. Indeed, compare to previous studies, see for example [6] and [50], and to anticipate meaningful modeling assumptions, we have considered at the microscopic level that the electric conductivities are tensorial. This does not appear in the expression of System (93) but is hidden in the definition of the cell-problems (88) and in the definition of the tensor (89). Moreover, we have shown that the classical macroscopic bidomain model formally obtained in [17] and [43] is valid under some more general conditions on the ionic terms than those assumed in [6] and [50]. More precisely, we have extended the validity of the macroscopic bidomain equations to space-varying physiological models.

\section{References}

[1] E. Acerbi, V. ChiadòPiat, G. Dal Maso, and Percivale D. An extension theorem from connected sets, and homogenization in general periodic domains. Nonlinear Analysis: Theory, Methods 83 Applications, 18(5):481—496, 1992.

[2] R. Aliev and A. Panfilov. A simple two-variable model of cardiac excitation. Chaos, Solitons \& Fractals, 3(7):293-301, 1996.

[3] G. Allaire. Homogenization and two-scale convergence. SIAM Journal of Mathematical Analysis, 23(6):1482-1518, 1992.

[4] G. Allaire, A. Damlamian, and U. Hornung. Two-scale convergence on periodic surfaces and applications. In Proceedings of the International Conference on Mathematical Modelling of Flow through Porous Media, 1995.

[5] G. Allaire and F. Murat. Homogenization of the Neumann problem with nonisolated holes. Asymptotic Analysis, 7(2):81-95, 1993.

[6] L. Ambrosio, P. Colli Franzone, and G. Savaré. On the asymptotic behaviour of anisotropic energies arising in the cardiac bidomain model. Interfaces and Free Boundaries, 2(3):213-266, 2000.

[7] H. Ammari, J. Garnier, L. Giovangigli, W. Jing, and J.-K. Seo. Spectroscopic imaging of a dilute cell suspension. Journal de Mathématiques Pures et Appliquées, 105(5):603$661,2016$.

[8] M. Bendahmane and H.K. Karlsen. Analysis of a class of degenerate reaction-diffusion systems and the bidomain model of cardiac tissue. Networks and Heterogeneous Media, 1:185-218, 2006. 
[9] A. Bensoussan, J.-L. Lions, and G. Papanicolaou. Asymptotic Analysis for Periodic Structures. Elsevier, 1978.

[10] M. Boulakia, M.A. Fernández, J-F. Gerbeau, and N. Zemzemi. A coupled system of PDEs and ODEs arising in electrocardiograms modeling. Applied Mathematics Research eXpress, 2008.

[11] Y. Bourgault, Y. Coudière, and C. Pierre. Existence and uniqueness of the solution for the bidomain model used in cardiac electrophysiology. Nonlinear Analysis-Real World Applications, 10(1):458-482, 2009.

[12] M. Briane. Three models of non periodic fibrous materials obtained by homogenization. RAIRO-Modélisation mathématique et analyse numérique, 27(6):759-775, 1993.

[13] D. Cioranescu, A. Damlamian, P. Donato, G. Griso, and R. r. Zaki. The periodic unfolding method in domains with holes. SIAM Journal on Mathematical Analysis, $4(2): 718-760,2010$.

[14] D. Cioranescu and J. Saint Jean Paulin. Homogenization in open sets with holes. Journal of Mathematical Analysis and Applications, 71(2):590 - 607, 1979.

[15] D. Cioranescu and J. Saint Jean Paulin. Homogenization of Reticulated Structures. Springer New York, 1999.

[16] P. Colli Franzone, L.F. Pavarino, and S. Scacchi. Mathematical Cardiac Electrophysiology, volume 13 of Modeling, Simulation and Applications. Springer International Publishing, 2014.

[17] P. Colli Franzone and G. Savaré. Degenerate evolution systems modeling the cardiac electric field at micro and macroscopic level. Progress in Nonlinear Differential Equations and Their Applications, 50:49-78, 2002.

[18] C. Corrado, J.-F. Gerbeau, and P. Moireau. Identification of weakly coupled multiphysics problems. application to the inverse problem of electrocardiology. Journal of Computational Physics, 2014. In review.

[19] M. Courtemanche, R.J. Ramirez, and S. Nattel. Ionic mechanisms underlying human atrial action potential properties: insights from a mathematical model. American Journal of Physiology, 275:301-321, 1998.

[20] K. Djabella, M. Landau, and M. Sorine. A two-variable model of cardiac action potential with controlled pacemaker activity and ionic current interpretation. In Decision and Control, 2007 46th IEEE Conference on, pages 5186-5191. IEEE, 2007.

[21] S.S. Dragomir. Some Gronwall type inequalities and applications. Nova Science Pub Incorporated, 2003.

[22] Lawrence C. Evans. Partial differential equations. American Mathematical Society, Providence, R.I., 2010.

[23] R. Fitzhugh. Impulses and physiological states in theoretical models of nerve membrane. Biophysical Journal, 1(6):445-466, 1961.

[24] S. Göktepe and E. Kuhl. Electromechanics of the heart: a unified approach to the strongly coupled excitation-contraction problem. Computational Mechanics, 45(2):227$243,2010$.

[25] E. Grandi, S.V. Pandit, N. Voigt, A.J. Workman, D. Dobrev, and J. Jalife. Human atrial action potential and $\mathrm{Ca} 2+$ model: sinus rhythm and chronic atrial fibrillation. Circ. Res., 109:1055-1066, 2011.

[26] E. Grandi, F.S. Pasqualini, and D M. Bers. A novel computational model of the human ventricular action potential and Ca transient. J Mol Cell Cardiol, 48:112-121, 2010.

[27] P.E. Hand and C.S. Peskin. Homogenization of an electrophysiological model for a strand of cardiac myocytes with gap-junctional and electric-field coupling. Bulletin of Mathematical Biology, 72:1408-1424, 2010. 
[28] A.L. Hodgkin and A.F. Huxley. A quantitative description of membrane current and its application to conduction and excitation in nerve. Journal of Physiology, 117(4):500$544,1952$.

[29] O. Kavian, M. Leguèbe, C. Poignard, and L. Weynans. "Classical" electropermeabilization modeling at the cell scale. Journal of mathematical biology, 68(1-2):235-265, 2014.

[30] J. Keener and J. Sneyd. Mathematical Physiology. Springer, 2004.

[31] J.T. Koivumäki, T. Korhonen, and P. Tavi. Impact of sarcoplasmic reticulum calcium release on calcium dynamics and action potential morphology in human atrial myocytes: A computational study. PLoS Computational Biology, 7(1):e1001067, 2011.

[32] K. Kunisch and A. Marica. Well-posedness for the Mitchell-Schaeffer model of the cardiac membrane. Research report, Institut für Mathematik, Karl-Franzens-Universität Graz, 2013.

[33] J.-L. Lions. Quelques méthodes de résolution des problèmes aux limites non linéaires, volume 31. Dunod Paris, 1969.

[34] J.-L. Lions and E. Magenes. Non-Homogeneous Boundary Value Problems and Applications, volume 1. Springer Berlin Heidelberg, 1972.

[35] C.H. Luo and Y. Rudy. A dynamic model of the cardiac ventricular action potential. I. Simulations of ionic currents and concentration changes. Circulation Research, 74:1071-1096, 1994.

[36] M.M. Maleckar, J.L. Greenstein, N.A. Trayanova, and W R. Giles. Mathematical simulations of ligand-gated and cell-type specific effects on the action potential of human atrium. Progress in biophysics and molecular biology, 98(2):161-170, 2008.

[37] Q. Marciniak-Czochra and M. Ptashnyk. Derivation of a macroscopic receptor-based model using homogenization techniques. SIAM Journal on Mathematical Analysis, 40(1):215 - 237, 2008.

[38] W. McLean. Strongly Elliptic systems and Boundary Integral equation. Cambridge University Press, Cambridge, 2000.

[39] C.C. Mitchell and D.G. Schaeffer. A two-current model for the dynamics of cardiac membrane. Bulletin Math. Bio., 65:767-793, 2003.

[40] Y. Mori. From three-dimensional electrophysiology to the cable model: an asymptotic study. arXiv preprint arXiv:0901.3914, 2009.

[41] Y. Mori, J.W. Jerome, and C.S. Peskin. A three-dimensional model of cellular electrical activity. Bulletin of the Institute of Mathematics, Academia Sinica, 2(2):367-390, 2007.

[42] J.S. Nagumo, S. Arimoto, and S. Yoshizawa. An active pulse transmission line stimulating nerve axon. Proceedings of the IEEE, 50:2061-2071, 1962.

[43] J.C. Neu and W. Krassowska. Homogenization of syncytial tissues. Critical Reviews in Biomedical Engineering, 21(2):137-199, 1993.

[44] Maria Neuss-Radu. Some extensions of two-scale convergence. Comptes rendus de l'Académie des sciences. Série 1, Mathématique, 322(9):899-904, 1996.

[45] G. Nguetseng. A general convergence result for a functional related to the theory of homogenization. SIAM Journal on Mathematical Analysis, 20(3):608 - 623, 1989.

[46] D. Noble. A modification of the Hodgkin-Huxley equation applicable to Purkinje fiber action and pacemaker potentials. Journal of Physiology, 160:317-352, 1962.

[47] A. Nygren, C. Fiset, L. Firek, J.W. Clark, D.S. Lindblad, R.B. Clark, and W.R. Giles. Mathematical model of an adult human atrial cell the role of $\mathrm{K}+$ currents in repolarization. Circulation Research, 82(1):63-81, 1998.

[48] T. O'Hara, L. Virág, A. Varró, and Y. Rudy. Simulation of the undiseased human cardiac ventricular action potential: Model formulation and experimental validation. PLoS Comput Biol, 7(5):e1002061, 2011. 
[49] D. O'Regan. Existence Theory for Nonlinear Ordinary Differential Equations. Springer Netherlands, 1997.

[50] M. Pennacchio, G. Savaré, and P. Colli Franzone. Multiscale modeling for the bioelectric activity of the heart. SIAM Journal on Mathematical Analysis, 37(4):1333-1370, 2005.

[51] Mariya Ptashnyk. Multiscale modelling and analysis of signalling processes in tissues with non-periodic distribution of cells. Vietnam Journal of Mathematics, 45(1):295-316, 2017.

[52] A.J. Pullan, M.L. Buist, and L.K. Cheng. Mathematically Modeling the Electrical Activity of the Heart. World Scientific, 2005.

[53] G. Richardson and J. Chapman. Derivation of the bidomain equations for a beating heart with a general microstructure. SIAM Journal on Applied Mathematics, 71(3):657$675,2011$.

[54] M. Rioux and Y. Bourgault. A predictive method allowing the use of a single ionic model in numerical cardiac electrophysiology. Mathematical Modelling and Numerical Analysis, 47:987-1016, 2013.

[55] J.-M. Rogers and A.-D. McCulloch. A collocation-Galerkin finite element model of cardiac action potential propagation. IEEE Transactions on Biomedical Engineering, 41(8):743-757, 1994.

[56] F.B. Sachse. Computational Cardiology: Modeling of Anatomy, Electrophysiology and Mechanics. Springer-Verlag, 2004.

[57] S. Sanfelici. Convergence of the Galerkin approximation of a degenerate evolution problem in electrocardiology. Numerical Methods for Partial Differential Equations, 18(2):218-240, 2002.

[58] R.E. Showalter. Monotone operators in Banach space and nonlinear partial differential equations, volume 49. American Mathematical Soc., 2013.

[59] J. Sundnes, G.T. Lines, X. Cai, B.F. Nielsen, K.A. Mardal, and A. Tveito. Computing the Electrical Activity in the Heart, volume 1 of Monographs in Computational Science and Engineering. Springer-Verlag, 2006.

[60] K.H.W.J. ten Tusscher, D. Noble, P.J. Noble, and A.V. Panfilov. A model for human ventricular tissue. American Journal of Physiology-Heart and Circulatory Physiology, 286(4):H1573-H1589, 2004.

[61] K.H.W.J. ten Tusscher and A.V. Panfilov. Alternans and spiral breakup in a human ventricular tissue model. American Journal of Physiology-Heart and Circulatory Physiology, 291(3):H1088-H1100, 2006.

[62] L. Tung. A bi-domain model for describing ischemic myocardial d-c potentials. $\mathrm{PhD}$ thesis, Massachusetts Institute of Technology. Dept. of Electrical Engineering and Computer Science., 1978.

[63] M. Veneroni. Reaction-diffusion systems for the microscopic bidomain model of the cardiac electric field. Mathematical methods in the applied sciences, 29(14):1631-1661, 2006.

[64] M. Veneroni. Reaction-diffusion systems for the macroscopic bidomain model of the cardiac electric field. Nonlinear Analysis: Real World Applications, 10(2):849-868, 2009.

[65] M. Wilhelms, H. Hettmann, M.M.C. Maleckar, J.T. Koivumäki, O. Dössel, and G. Seemann. Benchmarking electrophysiological models of human atrial myocytes. Frontiers in Physiology, 3(487), 2013.

\section{Appendix (proofs of Section 3.3)}

In what follows, we will work with the following weighted norm,

$$
\|\mid\| v\left\|_{t, \lambda}^{2}:=\int_{0}^{t} e^{-\lambda s}\right\| v(\cdot, s) \|_{L^{2}\left(\Gamma_{m}\right)}^{2} d s, \forall v \in L^{2}\left((0, T) \times \Gamma_{m}\right) .
$$


Note that this weighted norm is obviously equivalent to the standard $L^{2}$-norm. The parameter $\lambda$ is chosen later to obtain existence and uniqueness results. Given any function $U \in C^{0}\left([0, T] ; L^{2}\left(\Gamma_{m}\right)\right)$, we have the following lemma.

Lemma 2 If Assumptions 2, 4, 5 and 7a hold then there exists a unique function

$$
w_{U} \in C^{1}\left([0, T] ; L^{2}\left(\Gamma_{m}\right)\right)
$$

which is a solution of

$$
\begin{cases}\partial_{t} w_{U}+g\left(U, w_{U}\right)=0, & \Gamma_{m}, \forall t \in[0, T] \\ w_{U}(\vec{x}, 0)=w^{0}(\vec{x}), & \Gamma_{m}\end{cases}
$$

Moreover if Assumptions 9 and 10 are satisfied then for all $t \in[0, T]$ and almost all $\vec{x} \in \Gamma_{m}$,

$$
w_{\min } \leq w_{U}(\vec{x}, t) \leq w_{\max }
$$

Proof. By density of continuous functions in $L^{2}$ spaces, there exist two sequences $\left\{w_{n}^{0}\right\} \subset$ $C^{0}\left(\Gamma_{m}\right)$ and $\left\{U_{n}\right\} \subset C^{0}\left([0, T] \times \Gamma_{m}\right)$ such that

$$
\left\|w_{n}^{0}-w^{0}\right\|_{L^{2}\left(\Gamma_{m}\right)} \underset{n \rightarrow+\infty}{\longrightarrow} 0
$$

and for all $t \in[0, T]$,

$$
\left\|U_{n}(t)-U(t)\right\|_{L^{2}\left(\Gamma_{m}\right)} \underset{n \rightarrow+\infty}{\longrightarrow} 0 .
$$

Then for all $\vec{x} \in \Gamma_{m}$, we denote by $w_{U_{n}}(\vec{x}, \cdot)$, the solution of the following Cauchy problem (now $\vec{x}$ plays the role of a parameter), for all $\vec{x} \in \Gamma_{m}$ and $t \in[0, T]$,

$$
\left\{\begin{array}{l}
\frac{d}{d t} w_{U_{n}}(\vec{x}, \cdot)+g\left(\vec{x}, U_{n}(\vec{x}, \cdot), w_{U_{n}}(\vec{x}, \cdot)\right)=0 \\
w_{U_{n}}(\vec{x}, 0)=w^{0}(\vec{x})
\end{array}\right.
$$

Since we have assumed that $g$ is Lipschitz in its second argument by a standard application of the Picard-Lindelöf theorem, we can show that there exists a unique solution $w_{U_{n}}(\vec{x}, \cdot)$ to this problem which belongs to $C^{1}([0, T])$. Now, for all $x \in \Gamma_{m}$, for all $(n, m) \in \mathbb{N}^{2}$, we have,

$$
\frac{d}{d t}\left[w_{U_{n}}(\vec{x}, \cdot)-w_{U_{m}}(\vec{x}, \cdot)\right]=g\left(U_{m}(\vec{x}, \cdot), w_{U_{m}}(\vec{x}, \cdot)\right)-g\left(\vec{x}, U_{n}(\vec{x}, \cdot), w_{U_{n}}(\vec{x}, \cdot)\right) .
$$

We set $w_{n, m}:=w_{U_{n}}-w_{U_{m}}$. We multiply the previous equation by $e^{-\lambda t} w_{n, m}$ and integrate with respect to space and time. After some manipulations we get, for all $t \in[0, T]$,

$$
\begin{aligned}
\frac{e^{-\lambda t}}{2}\left\|w_{n, m}(t)\right\|_{L^{2}\left(\Gamma_{m}\right)}^{2}- & \frac{1}{2}\left\|w_{n}^{0}-w_{m}^{0}\right\|_{L^{2}\left(\Gamma_{m}\right)}^{2}+\frac{\lambda}{2}\left\|w_{n, m}\right\|_{t, \lambda}^{2} \\
& =\int_{0}^{t} e^{-\lambda s}\left[\left(g\left(U_{m}, w_{U_{m}}\right), w_{n, m}\right)_{\Gamma_{m}}-\left(g\left(U_{n}, w_{U_{n}}\right), w_{n, m}\right)_{\Gamma_{m}}\right] d s .
\end{aligned}
$$

Since $g$ is globally Lipschitz (Assumption 7a), we have

$\frac{e^{-\lambda t}}{2}\left\|w_{n, m}(t)\right\|_{L^{2}\left(\Gamma_{m}\right)}^{2} \leq\left(\frac{3 L_{g}}{2}-\frac{\lambda}{2}\right)\left\|\left|w_{n, m}\right|\right\|_{t, \lambda}^{2}+\frac{1}{2}\left\|w_{n}^{0}-w_{m}^{0}\right\|_{L^{2}\left(\Gamma_{m}\right)}^{2}+\frac{L_{g}}{2}\left\|\left|U_{n}-U_{m} \|\right|_{t, \lambda}^{2}\right.$.

Then choosing $\lambda>3 L_{g}$ in (96), we can deduce that $w_{U_{n}}$ is a bounded Cauchy sequence in $L^{2}\left((0, T) \times \Gamma_{m}\right)$ which is a Banach space. Therefore the sequence $w_{U_{n}}$ converges strongly to a limit denoted $w_{U}$. Moreover, since $U_{n}$ and $w_{U_{n}}$ converge strongly in $L^{2}\left((0, T) \times \Gamma_{m}\right)$ and since $g$ is Lipschitz (Assumption 7a), we have

$$
\left\||| g\left(U_{n}, w_{U_{n}}\right)-g\left(U(t), w_{U}\right)\right\|_{T, 0} \underset{n \rightarrow+\infty}{\longrightarrow} 0 .
$$


Then by passing to the limit in the weak formulation of (95), it can be proven that the limit $w_{U} \in L^{2}\left((0, T) \times \Gamma_{m}\right)$ is a weak solution of (94). In a second step, by inspection of (94), one can show that

$$
\frac{\partial w_{U}}{\partial t} \in L^{2}\left((0, T) \times \Gamma_{m}\right) .
$$

Therefore thanks to Lemma 1.2 of [33] - up to some modification on zero-measure sets the function $w_{U}$ satisfies

$$
w_{U} \in C^{0}\left([0, T] ; L^{2}\left(\Gamma_{m}\right)\right) .
$$

This last property implies, again by inspection of (35), that $w_{U} \in C^{1}\left([0, T] ; L^{2}\left(\Gamma_{m}\right)\right)$. Finally, by simple arguments and Assumption 9, the solution $w_{U_{n}}$ satisfies - for all $\vec{x} \in \Gamma_{m}$ and for all $t \in[0, T]-w_{\min } \leq w_{U_{n}}(\vec{x}, t) \leq w_{\max }$ if and only if

$$
w_{\min } \leq w_{n}^{0} \leq w_{\max } .
$$

This can be ensured for every $n$ and at the limit if it is satisfied for the initial data $w^{0}$ (i.e. Assumption 9 holds) and if we choose a sequence $\left\{w_{n}^{0}\right\}$ of approximating functions that preserves (97). Such sequences are classically constructed by convolution with parametrized smooth positive functions of measure one and of decreasing supports around the origin (see [38] Chapter 3 for instance).

Theorem 1 If Assumptions 1-7 hold, there exist

$$
V_{m} \in C^{0}\left([0, T] ; L^{2}\left(\Gamma_{m}\right)\right) \cap L^{2}\left((0, T) ; H^{1 / 2}\left(\Gamma_{m}\right)\right), \quad \partial_{t} V \in L^{2}\left((0, T) ; H^{-1 / 2}\left(\Gamma_{m}\right)\right),
$$

and

$$
w \in H^{1}\left((0, T) ; L^{2}\left(\Gamma_{m}\right)\right),
$$

which are solutions of

$$
\left\{\begin{array}{lll}
C_{m} \partial_{t} V_{m}+\mathcal{A}\left(V_{m}\right)+I_{i o n}\left(V_{m}, w\right)=I_{a p p}, & \widetilde{H}^{-1 / 2}\left(\Gamma_{m}\right), & \text { a.e. } t \in(0, T), \\
\partial_{t} w+g\left(V_{m}, w\right)=0, & \Gamma_{m}, & \text { a.e. } t \in(0, T),
\end{array}\right.
$$

and

$$
\begin{cases}V_{m}(\vec{x}, 0)=V_{m}^{0}(\vec{x}) & \Gamma_{m} \\ w(\vec{x}, 0)=w^{0}(\vec{x}) & \Gamma_{m}\end{cases}
$$

Proof. The proof uses the general ideas of the Faedo-Galerkin technique and some useful intermediate results which come from [33]. Our proof deals simultaneously with physiological and phenomenological models only if Assumption 7a is satisfied. Our proof is only partial when phenomenological models satisfying Assumption $7 \mathrm{~b}$ are considered. More precisely, the proof is valid up to Step 4 and we refer the reader to the analysis done in [11] and [10] to extend the proof.

Step 1: Introduction of the eigenvector basis of the operator $\mathcal{A}$.

We introduce $\left\{\lambda_{k}\right\}_{k \geq 0} \subset \mathbb{R}^{+}$and $\left\{\psi_{k}\right\}_{k \geq 0} \subset H^{1 / 2}\left(\Gamma_{m}\right)$, the set of increasing nonnegative eigenvalues and corresponding eigenvectors such that, for all $v \in H^{1 / 2}\left(\Gamma_{m}\right)$,

$$
\left\langle\mathcal{A}\left(\psi_{k}\right), v\right\rangle_{\Gamma_{m}}=\lambda_{k}\left(\psi_{k}, v\right)_{\Gamma_{m}} .
$$

Thanks to the properties of the operator $\mathcal{A}$ given by Proposition 3 and the fact that $H^{1 / 2}\left(\Gamma_{m}\right)$ is dense in $L^{2}\left(\Gamma_{m}\right)$ with compact injection, such eigenvalues and eigenvectors exist and the set $\left\{\psi_{k}\right\}_{k \geq 0}$ is an orthonormal basis of $L^{2}\left(\Gamma_{m}\right)$ (see [38], Theorem 2.37). Note that $\lambda_{0}=0$ and $\left.\psi_{0}=1 /\left|\Gamma_{m}\right|^{1 / 2}\right)$. As in [11], we introduce the continuous projection operator $\mathcal{P}_{\ell}: L^{2}\left(\Gamma_{m}\right) \rightarrow L^{2}\left(\Gamma_{m}\right)$ defined by

$$
\mathcal{P}_{\ell}(v)=\sum_{k=0}^{\ell}\left(v, \psi_{k}\right)_{\Gamma_{m}} \psi_{k} .
$$


It is standard to show that, for any $w \in L^{2}\left(\Gamma_{m}\right)$ and $v \in H^{1 / 2}\left(\Gamma_{m}\right)$,

$$
\lim _{\ell \rightarrow+\infty}\left\|w-\mathcal{P}_{\ell}(w)\right\|_{L^{2}\left(\Gamma_{m}\right)}=0 \quad \text { and } \quad \lim _{\ell \rightarrow+\infty}\left\|v-\mathcal{P}_{\ell}(v)\right\|_{H^{1 / 2}\left(\Gamma_{m}\right)}=0 .
$$

Finally, note that $\mathcal{P}_{\ell}$ is also continuous from $H^{1 / 2}\left(\Gamma_{m}\right)$ to $H^{1 / 2}\left(\Gamma_{m}\right)$ and

$$
\left\|\mathcal{P}_{\ell}(v)\right\|_{L^{2}\left(\Gamma_{m}\right)}^{2}=\sum_{k=0}^{\ell}\left|\left(v, \psi_{k}\right)_{\Gamma_{m}}\right|^{2}, \quad c\left\|\mathcal{P}_{\ell}(v)\right\|_{H^{1 / 2}\left(\Gamma_{m}\right)}^{2} \leq\left|\left(v, \psi_{0}\right)_{\Gamma_{m}}\right|^{2}+\sum_{k=1}^{\ell} \lambda_{k}\left|\left(v, \psi_{k}\right)_{\Gamma_{m}}\right|^{2}
$$

where $c>0$ is independent of $\ell$.

Step 2: Local existence result for a corresponding finite dimensional ODE system.

Multiplying the equations on $V_{m}$ and $w$ by $\psi_{k}$ and integrating over $\Gamma_{m}$ suggest to introduce for any given $\ell$ and for all $0 \leq k \leq \ell$ the following system of ordinary differential equations (ODE)

$$
\left\{\begin{array}{l}
C_{m} \frac{d}{d t} V_{k}^{\ell}+\lambda_{k} V_{k}^{\ell}+\int_{\Gamma_{m}} I_{i o n}\left(V^{\ell}, w^{\ell}\right) \psi_{k} d \gamma=\int_{\Gamma_{m}} I_{a p p} \psi_{k} d \gamma \\
\frac{d}{d t} w_{k}^{\ell}+\int_{\Gamma_{m}} g\left(V^{\ell}, w^{\ell}\right) \psi_{k} d \gamma=0
\end{array}\right.
$$

where we have defined

$$
V^{\ell}(\vec{x}, t):=\sum_{k=0}^{\ell} V_{k}^{\ell}(t) \psi_{k}(\vec{x}) \text { and } w^{\ell}(\vec{x}, t):=\sum_{k=0}^{\ell} w_{k}^{\ell}(t) \psi_{k}(\vec{x}) .
$$

The following initial conditions complete the system

$$
V_{k}^{\ell}(0):=V_{k}^{\ell, 0}=\mathcal{P}_{\ell}\left(V_{m}^{0}\right), \quad w_{k}^{\ell}(0):=w_{k}^{\ell, 0}=\mathcal{P}_{\ell}\left(w^{0}\right) .
$$

The idea is to apply standard existence results for an ODE system of the form

$$
\left\{\begin{array}{l}
\frac{d}{d t} V_{k}^{\ell}=i_{k}\left(t,\left\{V_{k}^{\ell}\right\},\left\{w_{k}^{\ell}\right\}\right), \\
\frac{d}{d t} w_{k}^{\ell}=g_{k}\left(t,\left\{V_{k}^{\ell}\right\},\left\{w_{k}^{\ell}\right\}\right), \\
V_{k}^{\ell}(0)=V_{k}^{\ell, 0}, \quad w_{k}^{\ell}(0)=w_{k}^{\ell, 0} .
\end{array}\right.
$$

where for all integers $0 \leq k \leq \ell$, the functions $i_{k}:[0, T] \times \mathbb{R}^{\ell} \times \mathbb{R}^{\ell} \rightarrow \mathbb{R}$ and $g_{k}:[0, T] \times$ $\mathbb{R}^{\ell} \times \mathbb{R}^{\ell} \rightarrow \mathbb{R}$ are defined by

$$
i_{k}\left(t,\left\{V_{k}^{\ell}\right\},\left\{w_{k}^{\ell}\right\}\right):=-\frac{\lambda_{k}}{C_{m}} V_{k}^{\ell}-\frac{1}{C_{m}} \int_{\Gamma_{m}} I_{i o n}\left(V^{\ell}, w^{\ell}\right) \psi_{k} d \gamma+\frac{1}{C_{m}} \int_{\Gamma_{m}} I_{a p p} \psi_{k} d \gamma,
$$

and

$$
g_{k}\left(t,\left\{V_{k}^{\ell}\right\},\left\{w_{k}^{\ell}\right\}\right):=-\int_{\Gamma_{m}} g\left(V^{\ell}, w^{\ell}\right) \psi_{k} d \gamma
$$

Such functions are continuous in each $V_{k}^{\ell}$ and $w_{k}^{\ell}$ thanks to Lemma 1.3 of [33] (see also the proof of Proposition 11). Moreover from Assumption 5 (i.e. $I_{i o n}$ and $g$ bounds) and from Assumption 3 (i.e. $I_{a p p} \in L^{2}\left((0, T) \times \Gamma_{m}\right)$ ), one can show that there exist positive scalars $C_{\ell}$ and $\widetilde{C}_{\ell}$ (depending on $\ell$ ) such that

$$
\begin{aligned}
& \sum_{k=0}^{\ell}\left|i_{k}\left(t,\left\{V_{k}^{\ell}\right\},\left\{w_{k}^{\ell}\right\}\right)\right|^{2}+\sum_{k=0}^{\ell} \mid\left.g_{k}\left(t,\left\{V_{k}^{\ell}\right\},\left\{w_{k}^{\ell}\right\}\right)\right|^{2} \\
& \leq C_{\ell}\left(\left\|V^{\ell}\right\|_{H^{1 / 2}\left(\Gamma_{m}\right)}^{2}+\left\|w^{\ell}\right\|_{L^{2}\left(\Gamma_{m}\right)}^{2}+1\right)+\left\|I_{a p p}(t)\right\|_{L^{2}\left(\Gamma_{m}\right)}^{2} . \\
& \leq \widetilde{C}_{\ell}\left(\sum_{k=0}^{\ell}\left|V_{k}^{\ell}\right|^{2}+\sum_{k=0}^{\ell}\left|w_{k}^{\ell}\right|^{2}+1\right)+\sup _{t \in[0, T]}\left\|I_{a p p}(t)\right\|_{L^{2}\left(\Gamma_{m}\right)}^{2} .
\end{aligned}
$$


This implies that $\left\{i_{k}, g_{k}\right\}$ are $L^{2}$-Carathéodory functions (see Definition 3.2 of [49]). Then, one can apply Theorem 3.7 of [49] to show that there exist $T_{0} \in[0, T]$ and solutions $V_{k}^{\ell}, w_{k}^{\ell}$ of Equation (100) such that

$$
V_{k}^{\ell} \in H^{1}\left(0, T_{0}\right), \quad w_{k}^{\ell} \in H^{1}\left(0, T_{0}\right), \quad 0 \leq k \leq \ell .
$$

Step 3: Existence result on $[0, T]$ for the finite dimensional ODE system.

Theorem 3.8 of [49] shows that $T_{0}>0$ is independent of the initial data. Now, our objective is to show that one can find a bound independent of $T_{0}$ on the solution for $t \in\left[0, T_{0}\right]$. Then such a uniform bound is used to guaranty existence of solutions up to a time $T \geq T_{0}$ by a recursion argument. By using an energy technique, one can deduce

$$
\begin{array}{r}
\frac{e^{-\lambda t} C_{m}}{2}\left\|V^{\ell}(t)\right\|_{L^{2}\left(\Gamma_{m}\right)}^{2}-\frac{C_{m}}{2}\left\|V^{\ell}(0)\right\|_{L^{2}\left(\Gamma_{m}\right)}^{2}+\frac{\lambda C_{m}}{2}\left\|V^{\ell}\right\|_{t, \lambda}^{2}=-\int_{0}^{t} e^{-\lambda s}\left\langle\mathcal{A} V^{\ell}, V^{\ell}\right\rangle d s \\
-\int_{0}^{t} e^{-\lambda s}\left[\left(I_{i o n}\left(V^{\ell}, w^{\ell}\right)-I_{a p p}, V^{\ell}\right)_{\Gamma_{m}}\right] d s .
\end{array}
$$

Furthermore, for $\mu>0$ as in (30) of Assumption 6, we have

$$
\frac{e^{-\lambda t} \mu}{2}\left\|w^{\ell}(t)\right\|_{L^{2}\left(\Gamma_{m}\right)}^{2}+\frac{\lambda \mu}{2}\left\|w^{\ell} \mid\right\|_{t, \lambda}^{2}=\frac{\mu}{2}\left\|w^{\ell}(0)\right\|_{L^{2}\left(\Gamma_{m}\right)}^{2}-\mu \int_{0}^{t} e^{-\lambda s}\left(g\left(V^{\ell}, w^{\ell}\right), w^{\ell}\right)_{\Gamma_{m}} d s .
$$

Summing the two previous equations and using (30), we get

$$
\begin{array}{r}
\frac{e^{-\lambda t} C_{m}}{2}\left\|V^{\ell}(t)\right\|_{L^{2}\left(\Gamma_{m}\right)}^{2}+\frac{e^{-\lambda t} \mu}{2}\left\|w^{\ell}(t)\right\|_{L^{2}\left(\Gamma_{m}\right)}^{2}+\int_{0}^{t} e^{-\lambda s}\left\langle\mathcal{A} V^{\ell}, V^{\ell}\right\rangle_{\Gamma_{m}} d s \\
\leq \frac{C_{m}}{2}\left\|V^{\ell}(0)\right\|_{L^{2}\left(\Gamma_{m}\right)}^{2}+\frac{\mu}{2}\left\|w^{\ell}(0)\right\|_{L^{2}\left(\Gamma_{m}\right)}^{2}+\int_{0}^{t} e^{-\lambda s}\left(\left(I_{a p p}, V^{\ell}\right)_{\Gamma_{m}}+C_{I}\left|\Gamma_{m}\right|\right) d s \\
\quad+\left(C_{I}-\frac{\lambda C_{m}}{2}\right)\left\|V^{\ell}\right\|_{t, \lambda}^{2}+\left(C_{I}-\frac{\lambda \mu}{2}\right)\|\| w^{\ell} \mid \|_{t, \lambda}^{2} .
\end{array}
$$

Choosing $\lambda$ large enough, we can show using Gronwall's inequality (see also Proposition 5) that there exists a constant $C_{T}$ that depends only on $C_{m}, \mu, C_{I}$ and $T$ such that, for all $t \leq T_{0}$

$$
\begin{aligned}
& \left\|V^{\ell}(t)\right\|_{L^{2}\left(\Gamma_{m}\right)}^{2}+\left\|w^{\ell}(t)\right\|_{L^{2}\left(\Gamma_{m}\right)}^{2}+\int_{0}^{t} e^{-\lambda s}\left\langle\mathcal{A} V^{\ell}, V^{\ell}\right\rangle_{\Gamma_{m}} d s \\
& \quad \leq C_{T}\left(\left\|V_{\ell}(0)\right\|_{L^{2}\left(\Gamma_{m}\right)}^{2}+\left\|w_{\ell}(0)\right\|_{L^{2}\left(\Gamma_{m}\right)}^{2}+\int_{0}^{T} e^{-\frac{\lambda}{2} t}\left\|I_{\text {app }}\right\|_{L^{2}\left(\Gamma_{m}\right)} d t+1\right) .
\end{aligned}
$$

Estimate (103) shows that the solution remains bounded up to time $T_{0}$. The bound being independent of $T_{0}$, one can repeat the process with initial data $V^{\ell}\left(T_{0}\right)$ and $w^{\ell}\left(T_{0}\right)$ and therefore construct a solution up to time $2 T_{0}$ (since $T_{0}$ is independent of the initial data). Such a solution satisfies (103) with initial data corresponding to $V_{\ell}\left(T_{0}\right)$ and $w_{\ell}\left(T_{0}\right)$. By repeating this process, we can construct a solution up to time $T$.

\section{Step 4: Strong convergence result for the potential}

First from (103) and from the coercivity of $\mathcal{A}$ (see (21)), we can deduce that there exists $C>0$ independent of $\ell$ such that

$$
\int_{0}^{T}\left\|V^{\ell}(t)\right\|_{H^{1 / 2}\left(\Gamma_{m}\right)}^{2} d t \leq C
$$


One can see that $V^{\ell}$ satisfies, for all $v \in H^{1 / 2}\left(\Gamma_{m}\right)$,

$$
\begin{aligned}
C_{m}\left\langle\partial_{t} V^{\ell}, v\right\rangle_{\Gamma_{m}}=C_{m}\left\langle\partial_{t} V^{\ell}\right. & \left., \mathcal{P}_{\ell}(v)\right\rangle_{\Gamma_{m}} \\
& =-\left\langle\mathcal{A}\left(V^{\ell}\right), \mathcal{P}_{\ell}(v)\right\rangle_{\Gamma_{m}}-\left\langle I_{i o n}\left(V^{\ell}, w^{\ell}\right)-I_{a p p}, \mathcal{P}_{\ell}(v)\right\rangle_{\Gamma_{m}}
\end{aligned}
$$

Using the continuity of $\mathcal{A}$ (Eq. (20)), the continuity of $\mathcal{P}_{\ell}(v)$ in $H^{1 / 2}\left(\Gamma_{m}\right)$, the bound on $I_{\text {ion }}$ (28), and the estimates (103)-(104), there exists another positive scalar $C$ independent of $\ell$ such that

$$
\int_{0}^{T}\left\|\partial_{t} V^{\ell}(t)\right\|_{\widetilde{H}^{-1 / 2}\left(\Gamma_{m}\right)}^{2} d t \leq C
$$

From these observations, we deduce that $\left\{V^{\ell}\right\}_{\ell}$ is bounded in

$$
\mathcal{Q}:=\left\{v \in L^{2}\left((0, T) ; H^{1 / 2}\left(\Gamma_{m}\right)\right), \frac{\partial v}{\partial t} \in L^{2}\left((0, T) ; \widetilde{H}^{-1 / 2}\left(\Gamma_{m}\right)\right)\right\} .
$$

Therefore, using the Lions-Aubin compactness theorem introduced in [33] (translated into english in [58]), we know that the space $\mathcal{Q}$ is included in the space $L^{2}\left((0, T) \times \Gamma_{m}\right)$ with compact injection. As a consequence, there exists $V_{m} \in \mathcal{Q}$ such that, up to a subsequence, $V^{\ell}$ converges weakly to $V_{m}$ in the space $L^{2}\left((0, T) ; H^{1 / 2}\left(\Gamma_{m}\right)\right)$ and $\partial_{t} V^{\ell}$ converges weakly to $\partial_{t} V_{m}$ in $L^{2}\left(0, T ; \widetilde{H}^{-1 / 2}\left(\Gamma_{m}\right)\right)$. Moreover, we have

$$
\lim _{\ell \rightarrow+\infty}\|\| V^{\ell}(t)-V_{m}(t)\|\|_{T, 0}=0 .
$$

Finally from [34] (Chapter 1 Theorem 3.2 or Chapter 3 Theorem 4.1), we know that

$$
V_{m} \in \mathcal{Q} \Rightarrow V_{m} \in C^{0}\left([0, T] ; L^{2}\left(\Gamma_{m}\right)\right) .
$$

We now want to identify the equations satisfied by the limit terms $w$ and $V_{m}$ in Steps 5 and 6 respectively. These steps have to be adapted in the case where Assumption $7 \mathrm{~b}$ holds instead of $7 \mathrm{a}$.

Step 5a: Strong convergence result and identification of the limit evolution equation for the gating variable

Since we have assumed that $g$ is globally Lipschitz, we can deduce a similar strong convergence result for the gating variable $w^{\ell}$. For all $\tilde{w} \in L^{2}\left(\Gamma_{m}\right)$, the equation satisfied by $w^{\ell}$ reads,

$\left(\partial_{t} w^{\ell}, \mathcal{P}_{\ell}(\tilde{w})\right)_{\Gamma_{m}}+\left(g\left(V^{\ell}, w^{\ell}\right), \mathcal{P}_{\ell}(\tilde{w})\right)_{\Gamma_{m}}=0 \quad \Leftrightarrow \quad\left(\partial_{t} w^{\ell}, \tilde{w}\right)_{\Gamma_{m}}+\left(g\left(V^{\ell}, w^{\ell}\right), \mathcal{P}_{\ell}(\tilde{w})\right)_{\Gamma_{m}}=0$,

We introduce the unique solution $w$ given by Lemma 2 with $U=V_{m}$. It is possible to show that the following equation is satisfied

$$
\left(\partial_{t} w-\partial_{t} w^{\ell}, \tilde{w}\right)_{\Gamma_{m}}+\left(g\left(V_{m}, w\right)-g\left(V^{\ell}, w^{\ell}\right), \tilde{w}\right)_{\Gamma_{m}}+\left(g\left(V^{\ell}, w^{\ell}\right), \tilde{w}-\mathcal{P}_{\ell}(\tilde{w})\right)_{\Gamma_{m}}=0
$$

Setting $\tilde{w}:=w-w^{\ell}$ (hence $\left.\tilde{w}-\mathcal{P}_{\ell}(\tilde{w})=w-\mathcal{P}_{\ell}(w)\right)$, we obtain for almost all time $t \in(0, T)$

$$
\frac{1}{2} \frac{d}{d t}\left\|w-w^{\ell}\right\|_{\Gamma_{m}}^{2}+\left(g\left(V_{m}, w\right)-g\left(V^{\ell}, w^{\ell}\right), w-w^{\ell}\right)_{\Gamma_{m}}=-\left(g\left(V_{m}, w\right), w-\mathcal{P}_{\ell}(w)\right)_{\Gamma_{m}}
$$

Since the right-hand side vanishes when $\ell$ tends to infinity and using the Lipschitz property of $g$, we can deduce by a standard energy technique that

$$
\lim _{\ell \rightarrow+\infty}\left\||| w-w^{\ell}\right\|_{\lambda, T}=0
$$

for $\lambda>0$ sufficiently large. 
Step 6a: Identification of the limit evolution equation for the potential

We have already shown that $w$ satisfies the equation for the gating variable. We now want to pass to the limit in the space-time weak form of (105) which reads: for all $v \in$ $C^{1}\left([0, T] ; H^{1 / 2}\left(\Gamma_{m}\right)\right)$ such that $v(T)=0$, we have

$$
\begin{aligned}
-C_{m} \int_{0}^{T}\left\langle\partial_{t} \mathcal{P}_{\ell}(v), V^{\ell}\right\rangle_{\Gamma_{m}} d t+\int_{0}^{T} & \left\langle\mathcal{A}\left(V^{\ell}\right), \mathcal{P}_{\ell}(v)\right\rangle_{\Gamma_{m}} d t+\int_{0}^{T}\left\langle I_{i o n}\left(V^{\ell}, w^{\ell}\right), \mathcal{P}_{\ell}(v)\right\rangle_{\Gamma_{m}} d t \\
& =\int_{0}^{T}\left\langle I_{a p p}, \mathcal{P}_{\ell}(v)\right\rangle_{\Gamma_{m}} d t-C_{m}\left(\mathcal{P}_{\ell}\left(V_{m}^{0}\right), v(0)\right)_{\Gamma_{m}} . \quad(110)
\end{aligned}
$$

The first two terms pass to the limit - using the weak convergence of $V^{\ell}$ to $V_{m}$ in $\mathcal{Q}$ therefore,

$$
\lim _{\ell \rightarrow+\infty} \int_{0}^{T}\left\langle\partial_{t} \mathcal{P}_{\ell}(v), V^{\ell}\right\rangle_{\Gamma_{m}} d t=\lim _{\ell \rightarrow+\infty} \int_{0}^{T}\left\langle\partial_{t} v, V^{\ell}\right\rangle_{\Gamma_{m}} d t=\int_{0}^{T}\left\langle\partial_{t} v, V_{m}\right\rangle_{\Gamma_{m}} d t
$$

Furthermore, using Proposition 3 and the properties of the operator $\mathcal{P}_{\ell}$, we have

$$
\begin{aligned}
\lim _{\ell \rightarrow+\infty} \int_{0}^{T}\left\langle\mathcal{A}\left(V^{\ell}\right), \mathcal{P}_{\ell}(v)\right\rangle_{\Gamma_{m}} d t=\lim _{\ell \rightarrow+\infty} \int_{0}^{T}\left\langle\mathcal{A}\left(V^{\ell}\right), v\right\rangle_{\Gamma_{m}} d t \\
\quad=\lim _{\ell \rightarrow+\infty} \int_{0}^{T}\left\langle\mathcal{A}(v), V^{\ell}\right\rangle_{\Gamma_{m}} d t=\int_{0}^{T}\left\langle\mathcal{A}(v), V_{m}\right\rangle_{\Gamma_{m}} d t=\int_{0}^{T}\left\langle\mathcal{A}\left(V_{m}\right), v\right\rangle_{\Gamma_{m}} d t
\end{aligned}
$$

Using only the approximation properties of the operator $\mathcal{P}_{\ell}$, we find

$$
\lim _{\ell \rightarrow+\infty} \int_{0}^{T}\left\langle I_{a p p}, \mathcal{P}_{\ell}(v)\right\rangle_{\Gamma_{m}} d t-C_{m}\left(\mathcal{P}_{\ell}\left(V_{m}^{0}\right), v(0)\right)_{\Gamma_{m}}=\int_{0}^{T}\left\langle I_{a p p}, v\right\rangle_{\Gamma_{m}} d t-C_{m}\left(V_{m}^{0}, v(0)\right)_{\Gamma_{m}} .
$$

Finally, the last difficulty is to prove that the term $I_{i o n}\left(V^{\ell}, w^{\ell}\right)$ converges weakly to the term $I_{i o n}\left(V_{m}, w_{m}\right)$. Using Assumption 5 , there exists a scalar $C>0$ independent of $\ell$ such that

$$
\int_{0}^{T}\left\|I_{i o n}\left(V^{\ell}, w^{\ell}\right)\right\|_{L^{4 / 3}\left(\Gamma_{m}\right)}^{4 / 3} d t \leq C\left(\int_{0}^{T}\left\|V^{\ell}\right\|_{L^{4}\left(\Gamma_{m}\right)}^{4} d t+\int_{0}^{T}\left\|w^{\ell}\right\|_{L^{2}\left(\Gamma_{m}\right)}^{2} d t+1\right) .
$$

From Estimation (103) and the continuous injection $H^{1 / 2}\left(\Gamma_{m}\right) \subset L^{4}\left(\Gamma_{m}\right)$, we can deduce that $I_{i o n}\left(V^{\ell}, w^{\ell}\right)$ is uniformly bounded in $L^{4 / 3}\left((0, T) \times \Gamma_{m}\right)$. Moreover, since $V^{\ell}$ and $w^{\ell}$ converge almost everywhere to $V_{m}$ and $w$ respectively, $I_{i o n}\left(V^{\ell}, w^{\ell}\right)$ converges weakly to the term $I_{i o n}\left(V_{m}, w\right)$ in

$$
L^{4 / 3}\left((0, T) \times \Gamma_{m}\right) \subset L^{2}\left((0, T) ; \widetilde{H}^{-1 / 2}\left(\Gamma_{m}\right)\right),
$$

by application of Lemma 1.3 in [33] (see also the proof of Proposition 11). We can therefore deduce that $V_{m}$ satisfies the weak formulation of the bidomain equations. For all $v \in$ $C^{1}\left([0, T] ; H^{1 / 2}\left(\Gamma_{m}\right)\right)$ such that $v(T)=0$, we have

$$
\begin{array}{r}
-C_{m} \int_{0}^{T}\left\langle\partial_{t} v, V_{m}\right\rangle_{\Gamma_{m}} d t+\int_{0}^{T}\left\langle\mathcal{A}\left(V_{m}\right), v\right\rangle_{\Gamma_{m}} d t+\int_{0}^{T}\left\langle I_{i o n}\left(V_{m}, w\right), v\right\rangle_{\Gamma_{m}} d t \\
=\int_{0}^{T}\left\langle I_{a p p}, v\right\rangle_{\Gamma_{m}} d t-C_{m}\left(V_{m}^{0}, v(0)\right)_{\Gamma_{m}}
\end{array}
$$

Note that using the weak formulation, we see that the initial condition is $V_{m}(0)=V_{m}^{0}$. However, this has a meaning only if $V_{m}$ is continuous in $[0, T]$ with value $\sin L^{2}\left(\Gamma_{m}\right)$ which is the case (Equation (107)). Moreover, from the weak formulation (112), we deduce System (98) (in the sense of distribution in time). Finally, we deduce the regularity given in the statement of the theorem for $\partial_{t} V_{m}$. The regularity for $\partial_{t} w$ follows. 


\section{Remark 7. Energy identity for the limit solution}

To obtain an energy identity, observe that from the weak formulation (112)

$$
\begin{aligned}
C_{m} \int_{0}^{T}\left\langle\partial_{t} V_{m}, v\right\rangle_{\Gamma_{m}} d t+\int_{0}^{T}\left\langle\mathcal{A}\left(V_{m}\right), v\right\rangle_{\Gamma_{m}} d t+\int_{0}^{T}\left\langle I_{i o n}\left(V_{m}, w\right), v\right\rangle_{\Gamma_{m}} d t \\
=\int_{0}^{T}\left\langle I_{a p p}, v\right\rangle_{\Gamma_{m}} d t
\end{aligned}
$$

Then, all the terms above can be seen as linear forms on $v$ which are continuous in the norm of $L^{2}\left((0, T) ; H^{1 / 2}\left(\Gamma_{m}\right)\right)$. Therefore, by the density of functions in $C^{1}\left([0, T] ; H^{1 / 2}\left(\Gamma_{m}\right)\right)$ with compact support into $L^{2}\left((0, T) ; H^{1 / 2}\left(\Gamma_{m}\right)\right)$, Equation (113) is true with $v$ replaced by $e^{-\lambda t} V_{m}$. Finally, since $V_{m} \in C^{0}\left([0, T], L^{2}\left(\Gamma_{m}\right)\right)$, it can be shown that (see Theorem 3, Chapter 5 of [22] for some ideas of the proof),

$2 \int_{0}^{T}\left\langle\partial_{t} V_{m}, e^{-\lambda t} V_{m}\right\rangle_{\Gamma_{m}} d t=e^{-\lambda T}\left\|V_{m}(T)\right\|_{L^{2}\left(\Gamma_{m}\right)}^{2}-\left\|V_{m}(0)\right\|_{L^{2}\left(\Gamma_{m}\right)}^{2}+\lambda \int_{0}^{T} e^{-\lambda t}\left\|V_{m}\right\|_{L^{2}\left(\Gamma_{m}\right)}^{2} d t$.

From (113), we deduce

$$
\begin{array}{r}
\frac{C_{m}}{2} e^{-\lambda T}\left\|V_{m}(T)\right\|_{L^{2}\left(\Gamma_{m}\right)}^{2}-\frac{C_{m}}{2}\left\|V_{m}(0)\right\|_{L^{2}\left(\Gamma_{m}\right)}^{2}+\frac{\lambda C_{m}}{2} \int_{0}^{T} e^{-\lambda t}\left\|V_{m}\right\|_{L^{2}\left(\Gamma_{m}\right)}^{2} d t \\
+\int_{0}^{T} e^{-\lambda t}\left\langle\mathcal{A}\left(V_{m}\right), V_{m}\right\rangle_{\Gamma_{m}} d t+\int_{0}^{T} e^{-\lambda t}\left\langle I_{i o n}\left(V_{m}, w\right), V_{m}\right\rangle_{\Gamma_{m}} d t \\
=\int_{0}^{T} e^{-\lambda t}\left\langle I_{a p p}, V_{m}\right\rangle_{\Gamma_{m}} d t
\end{array}
$$

Moreover, from the evolution equation (98) on the gating variable $w$ and since

$$
w \in H^{1}\left([0, T] ; L^{2}\left(\Gamma_{m}\right)\right) \quad \text { and } \quad g\left(V_{m}, w\right) \in L^{2}\left([0, T] ; L^{2}\left(\Gamma_{m}\right)\right),
$$

we deduce straightforwardly the energy identity

$$
\begin{aligned}
\frac{\mu}{2} e^{-\lambda T}\|w(T)\|_{L^{2}\left(\Gamma_{m}\right)}^{2}-\frac{\mu}{2}\|w(0)\|_{L^{2}\left(\Gamma_{m}\right)}^{2}+\frac{\lambda \mu}{2} \int_{0}^{T} e^{-\lambda t}\|w\|_{L^{2}\left(\Gamma_{m}\right)}^{2} d t & \\
& +\mu \int_{0}^{T} e^{-\lambda t}\left(g\left(V_{m}, w\right), w\right)_{\Gamma_{m}} d t=0 .
\end{aligned}
$$

Summing (114) and (115), we get the fundamental energy identity.

Corollary 2 If Assumption 8 holds then the solution of the microscopic bidomain equation (36) given by Theorem 1 is unique.

Proof. The proof is standard. Indeed, we assume that two solutions exist and we show that they must be equal by the energy estimate. We denote by $\left(V_{1}, w_{1}\right)$ and $\left(V_{2}, w_{2}\right)$ two solutions of (36). Following the same way that we have used to obtain (114) and then (115), we have for $\left(V_{1}, V_{2}\right)$,

$$
\begin{aligned}
\frac{e^{-\lambda t} C_{m}}{2}\left\|V_{1}(t)-V_{2}(t)\right\|_{L^{2}\left(\Gamma_{m}\right)}^{2}+\frac{\lambda C_{m}}{2}\left\|V_{1}-V_{2}\right\| \|_{t, \lambda}^{2} \leq & -\int_{0}^{t} e^{-\lambda s}\left[\left\langleI_{i o n}\left(V_{1}, w_{1}\right)\right.\right. \\
& \left.\left.-I_{i o n}\left(V_{2}, w_{2}\right), V_{1}-V_{2}\right\rangle_{\Gamma_{m}}\right] d s
\end{aligned}
$$


and for $\left(w_{1}, w_{2}\right)$

$$
\begin{aligned}
\frac{e^{-\lambda t} \mu}{2}\left\|w_{1}(t)-w_{2}(t)\right\|_{L^{2}\left(\Gamma_{m}\right)}^{2}+\frac{\lambda \mu}{2}\left\|\mid w_{1}-w_{2}\right\| \|_{t, \lambda}^{2} \leq-\mu \int_{0}^{t} e^{-\lambda s}\left[\left\langleg\left(V_{1}, w_{1}\right)\right.\right. & \\
& \left.\left.-g\left(V_{2}, w_{2}\right), w_{1}-w_{2}\right\rangle_{\Gamma_{m}}\right] d s .
\end{aligned}
$$

Collecting the two previous equations and using the one-side Lipschitz assumption (Assumption 8), we find

$$
\begin{aligned}
C_{m} \| V_{1}(t)-V_{2}(t) & \left\|_{L^{2}\left(\Gamma_{m}\right)}^{2}+\mu\right\| w_{1}(t)-w_{2}(t) \|_{L^{2}\left(\Gamma_{m}\right)}^{2} \\
& \leq e^{\lambda t}\left(2 L_{I}-\lambda C_{m}\right)\|\| V_{1}-V_{2}\|\|_{t, \lambda}^{2}+e^{\lambda t}\left(2 L_{I}-\lambda \mu\right)\left\|\mid w_{1}-w_{2}\right\|_{t, \lambda}^{2} .
\end{aligned}
$$

Choosing $\lambda$ large enough, we obtain $V_{1}=V_{2}$ and $w_{1}=w_{2}$. 